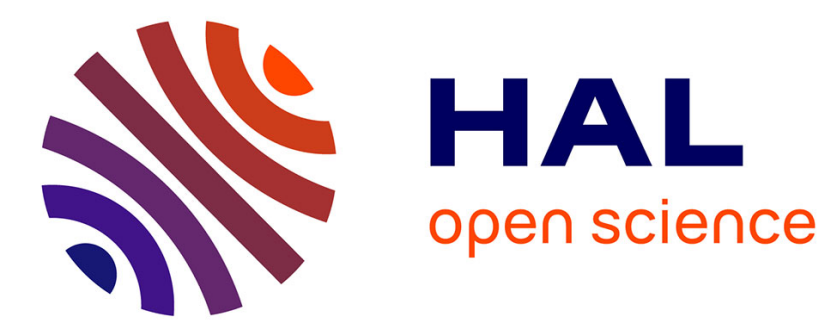

\title{
Orientation nucléaire par pompage optique des isotopes impairs du mercure
}

Bernard Cagnac

\section{To cite this version:}

Bernard Cagnac. Orientation nucléaire par pompage optique des isotopes impairs du mercure. Physique Atomique [physics.atom-ph]. Université Paris, 1960. Français. NNT ： · tel-00011795

\section{HAL Id: tel-00011795 \\ https://theses.hal.science/tel-00011795}

Submitted on 8 Mar 2006

HAL is a multi-disciplinary open access archive for the deposit and dissemination of scientific research documents, whether they are published or not. The documents may come from teaching and research institutions in France or abroad, or from public or private research centers.
L'archive ouverte pluridisciplinaire HAL, est destinée au dépôt et à la diffusion de documents scientifiques de niveau recherche, publiés ou non, émanant des établissements d'enseignement et de recherche français ou étrangers, des laboratoires publics ou privés. 


\title{
THESES
}

présentées

\section{A LA FACULTÉ DES SCIENCES DE L'UNIVERSITÉ DE PARIS}

Pour obtenir le grade de Docteur ès Sciences Physiques

par

\section{Bernard CAGNAC}

\author{
PREMIẼRE THÈSE \\ ORIENTATION NUCLEAIRE \\ PAR POMPAGE OPTIQUE \\ DES ISOTOPES IMPAIRS DU MERCURE
}

DFUXI亡̀MF，TH TSE

\section{PROPOSITIONS DONNÉES PAR LA FACULTÉ}

SOUTENUES LE DÉCEMBRE 1960 DEVANT LA COMMISSION D'EXAMEN

MM. Y. ROGARD, Président
A. KASTLER
P. JACQUINOT
Examinatenrs
J. BROSSEL 


\title{
TH E S E S \\ présentées
}

\section{A LA FACULTE' DES SCIENCES DE L'UNIVERSITE}

\author{
DE PARIS
}

pour obtenir le grade de Docteur ès Sciences Physiques

par

Bernard CAGNAC

Première Thèse : ORIEUTATION NUCLEATPE PAR FOMPAGE OPTIOUE DES ISOTOPES IVIPAIRS DU MERCURT.

Deuxième Thèse : Propositions données par la Faculté.

Soutenues le Décemure 1960 devant la Commission d'Exxumen :

JURY : M. Y. ROCARD, Président

PM. A. KASTILER )

P. JACQUTNOT) Exaninateurs

J. BROSSEL ) 


\section{- THBLE DES MLITIERES -}

INTRODUCTION

page 1

PREMIER CH $l_{2}$ PITRE - Les conditions du pompage optique.

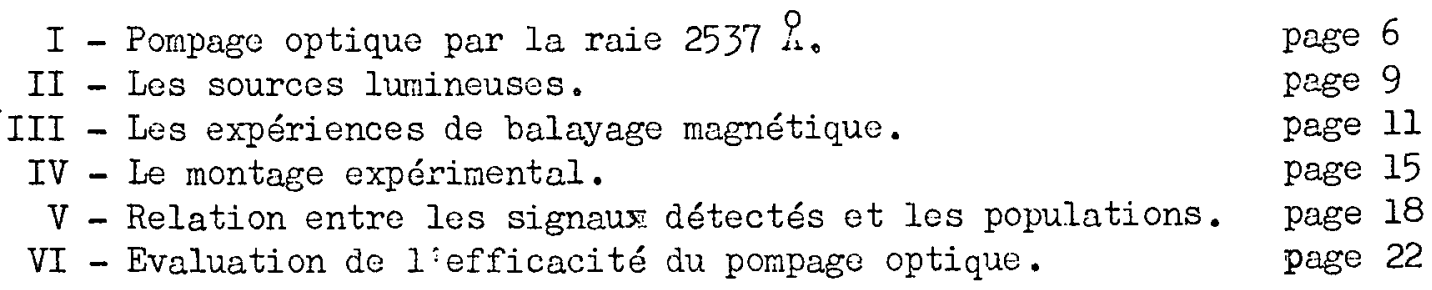

DEUXIEME CHAPITRE - Mesure des moments nucléaires.

VII - Mesures préliminaires à fréquence fixe. $\quad$ page 28

VIII - Contrôle du champ directeur H par résonance de protons. page 29

IX - Les bobines et le champ $\mathrm{H}_{1}$ de radiofréquence. page 31

$\mathrm{X}$ - Mesures en champ fixe.

XI - Lies moments magnétiques nucléaires.

page 33

page 35

TROISIENE CHAPITRE - Les méthodes d'étude de la relaxation.

XII - Les temps de relaxation et la méthode statique.

page 38

XIII - La méthode dynamique. Observation des transjtoires de

XIV - La méthode de calcul. $\mathrm{Hg}$.

page 42

page 48

$X V-C a s$ du $199 \mathrm{Hg}(\operatorname{spin} 1 / 2)$.

page 51

XVI - Cas du $201 \mathrm{Hg}$ en alignement。

page 56

XVII - Cas du ${ }^{201} \mathrm{Hg}$ en orientation.

page 62

QUATRIEME CHAPITRE - Les origines de la relaxation.

XVIII - Quelques contrôles des mesures de temps de relaxation.

page 64

XIX - Généralités sur les causes de relaxation.

$X X$ - Les inhomogénéi tés spatialess

XXI - Relaxation de $201_{\mathrm{Hg}}$.

XXII - Relaxation de ${ }^{199} \mathrm{Hg}$.

page 70

page 74

page 77

page 81

CONCLUSION

page 86

GNNEXE I

page 88

ANNEXE II

page 90

BIBLIOGR/PHIE

PIGURES 
Ce travail a été effectué au laboratoire de physique de I'E.N.S, durant les années 1957- 1960 sous la direction de Messieurs A. KASTLER et J. BROSSEI. Je Jes prio de trouver ici mes remerciements pour la confiance qu'ils miont accordée en me proposant ce sujet de thèse, et pour I'aide constante qu'ils m'ont apportée au cours de son étude.

Te remercie Monsieur Y. ROCARD, directeur du laboratoire, pour les facilités de travail que j'ai trouvées dans sa maison.

Je remercie tous les membres de l'équipe de résonance magnétiquo pour toutes les occasions où jiai pu apprécier leur aide et leur amitié, et spécialement J.P. BARRLT et C. COHEN-TANNOUDJI pour les discussions fructueuses que nous avons eues.

Jo remercie également les techniciens du laboratoire, dont les services aimables ct compétents ont grandement facilité ma tâche.

Ce travail oût été impossible sans los échantillons enrichis d'isotopes de mercure qui nous ont été fournis par le service de séparation isotopique du Commissariat à l'Energie Atomique. Qu'il on soit ici remercié. 


\section{ORIENTATION NUCLE'AIRE PAR POMPAGE OPTIQUE DES ISOTOPES}

\section{IMPAIRS DU MERCURE.}

\section{INTRODUCTION}

Le travail présenté ici est une application de la méthode de pompage optique suggérée par Kastler en 1950 (1). Cette méthode permet $d^{\prime}$ 'étendre aux états fondamentaux des atomes, par orientation de leurs moments magnétiques, la méthode de double résonance de Brossel et Kastler (2), appliquée d'abord aux états excités par Brossel (3). La première application du pompage optique fut réalisée en 1952 au laboratoire de l'Ecole Normale Supérieure sur le cas diu sodium (4). Elle se répandit ensuite dans de nombreux laboratoires étrangers où elle s'enrichit de nombreuses variantes (5). Mais elle n'avait été appliquée jusqu'ici qu'à des atomes alcalins, dont l'état fondamental est paramagnétique. Nous décrivons ici sa première application au cas d'atomes dont I'état fondamental est diamagnétique, mais qui possèdent un paramagnétisme nucléaire $(J=0 ; F=I)$; nous avons choisi comme exemple les isotopes impairs du mercure, ${ }^{199} \mathrm{Hg}$ de spin nucléaire $I=1 / 2$ et ${ }^{201} \mathrm{Hg}$ de spin nucléaire $I=3 / 2$ (état fondamental $6{ }^{1} \mathrm{~S}_{0}$ ). La méthode permet a'obtenir dans co cas une orientatinn purenent nucléaire.

La méthode de pompage optique a été souvent décrite (1)(6): on irradie une vapeur dans la direction du champ magnétique directeur $H_{0}$ avec sa raie de résonance polarisée circulairement, $\sigma^{+}$ par exemple; les atomes du sous-niveau Zeeman $m_{F}$ de l'état fonda- 
mental passent au sous-niveau $\mathrm{m}_{\mathrm{F}}^{\prime}=\mathrm{m}_{\mathrm{F}}+1$ de l'état excité; en retombant spontanément à l'état fondamental, ils se répartissent entre les sous-niveaux $\mathrm{m}_{\mathrm{F}}, \mathrm{m}_{\mathrm{F}}+1, \mathrm{~m}_{\mathrm{F}}+2$. Une part $\mathrm{du}$ moment angulaire des photons excitateurs se trouve ainsi transférée aux atomes; ceux-ci deviennent plus nombreux dans les sous-niveaux de grande valeur de $\mathrm{m}_{\mathrm{F}}$, c'est-à-dire que leurs moments magnétiques se trouvent partiellement orientés. Dans le cas où il y a plus de deux sous-niveaux ( $F \geqslant 1)$, l'irradiation en lumière naturelle suivant la direction du champ $\mathrm{H}_{\mathrm{O}}$ (lumière $\sigma$ ) peut conduire, selon les probabilités de transition optique, à vider symétriquement les sous-niveaux de faible valeur absolue $\left|\mathrm{m}_{\mathrm{F}}\right|$ au profit des sous-niveaux de grande valeur absolue $\left|\mathrm{m}_{\mathrm{F}}\right|$, c'est-à-dire que les moments magnétiques se trouvent partiellement alignés (7).

On peut obtenir ainsi de grandes différences de populations entre les sous-niveaux Zeeman de l'état fondamental, alors que, à l'équilibre thermique, la loi de Boltzmann conduit à des populations sensiblement égales (I'énergie Zeeman est négligeable devant $\mathrm{kT}$ ). Les phénomènes de relaxation tendent évidemment à rétablir cet équilibre thermique, par désorientation des atomes, avec une constante de temps égale au temps de relaxation Iongitudinale $\mathrm{T}_{1}$; et les différences de populations effectivement atteintes à l'état stationnaire résultent de la compétition entre ces phénomènes de relaxation et le pompage optique. Four qu'elles soient importantes, il faut que le cycle de pompage se reproduise à des intervalles de temps assez courts, comparés à $\mathrm{T}_{1}$; le temps moyen $\mathrm{T}_{\mathrm{p}}$ séparant l'absorption de deux photons par le même atome (inversement proportionnel à l'intensité Iumineuse) doit être du même ordre de grandeur ou plus court que $\mathrm{T}_{1}(8)$. 
L'irradiation lumineuse, qui sert à réaliser le pompage optique, joue également un second rôle, en permettant la détection des variations de populations. Le taux de polarisation (ou l'intensité) de la lumière de résonance optique réémise par la vapeur dépend en effet des populations relatives des sous-niveaux Zeeman de I'état fondamental. Une résonance magnétique à saturation entre deux tels sous-niveaux tend à égaliser leurs populations; et le changement de taux de polarisation (ou d'intensité) de la lumière réémise permet de détecter simultanément cette résonance magnétique et I'orientation préalable de la vapeur.

Dans le cas particulier d'atomes diamagnétiques, le temps $\mathrm{T}_{1}$ est un temps de relaxation nucléaire, qui doit être beaucoup plus long que les temps de relaxation électronique. On devait donc s'attendre à ce que I'orientation nucléaire fût plus facile à obtenir que l'orientation électronique. C'est la raison pour laquelle la méthode de pompage optique fut essayée pour la première fois par Brossel et Bitter (3)(9) précisément sur le mercure (isotope ${ }^{199} \mathrm{Hg}$ ); mais en fait, cette expérience fut un échec, et elle fut suivie d'autres essais négatifs (10), alors qu'on réussissait à observer successivement l'orientation des vapeurs de tous les métaux alcalins (11). On était conduit à penser que la cause de ces échecs tenait au manque d'intensité de la source lumineuse pompante sur la raie 2537 \& utilisée ( $T_{p}$ trop long).

Notre premier travail a été la mise au point de lampes à mercure émettant sur cette raie une forte intensité. Les résultats positifs ont immédiatement suivi, permettant d'observer dès 1958 l'alignement nucléaire de ${ }^{201} \mathrm{Hg}$ (12), puis I'orientation nucléaire de ${ }^{201} \mathrm{Hg}$ et ${ }^{199} \mathrm{Hg}$ (13). Nous décrivons d'abord dans le premier chapitre les caractéristiques de ces sources lumineuses, et les 
conditions exactes dans lesquelles nous opérions le pompage optique; nous y calculons ensuite les signaux que notre méthode de détection permet de mesurer en fonction des variations des populations des sous-niveaux Zeeman, et les maxima que l'on peut attendre en fonction du rapport $\mathrm{T}_{1} / \mathrm{T}_{\mathrm{p}}$ pour ces variations de populations.

Dans le deuxième chapitre, nous décrivons comment les courbes de résonance magnétique nucléaire observées nous ont permis la mesure précise des moments magnétiques nucléaires de ${ }^{201} \mathrm{Hg}$ et ${ }^{199} \mathrm{Hg}$; et comment on en déduit I'existence d'anomalies de structure hyperfine importantes dans les niveaux $6^{3} \mathrm{P}_{1}$ et $6^{3} \mathrm{P}_{2}$. Les taux d'orientation ou d'alignement mesurés ainsi que les largeurs de raie nous donnent déjà un ordre de grandeur des temps de relaxation, beaucoup plus longs pour ${ }^{199}$ Hig que pour ${ }^{201} \mathrm{Hg}$; mais les études détailiées des formes de raie et de la relaxation sont données dans les chapitres suivants.

Dans le troisième chapitre, nous décrivons en détail les deux méthodes employées pour l'étude de la relaxation. La première, que nous appelons méthode statique, utilise de façon tout à fait classique les mesures des largeurs des courbes de résonance, et permet une mesure assez précise du temps de relaxation transversale $\mathrm{T}_{2}$. La seconde méthode, que nous appelons méthode dynanique, utilise liobservation des transitoires du pompage optique et de la résonance magnétique, et permet la mesure précise des deux temps de relaxation, longitudinale $\mathrm{T}_{1}$, et transversale $\mathrm{T}_{2}$; l'utilisation de cette seconde méthode n'est facile que si les temps de relaxation sont assez longs, c'est-à-dire dans le cas de ${ }^{199} \mathrm{Hg}$. Les formules données avec ces deux méthodes sont justifiées à la fin du troisième chapitre par les calculs détaillés de l'évolution 
des populations sous l'action simultanée du champ de radiof réquence et de l'irradiation lumineuse; nous utilisons pour ces calculs le formalisme de la matrice densité.

Nous étudions enfin dans le quatrième chapitre les origines de la relaxation. Nous étudions $d^{\prime}$ abord le cas de ${ }^{201}$ Hg qui s'avère le plus simple en première approximation grâce à l'existence d'un mécanisme prédominant lié au moment quadrupolaire de ${ }^{201} \mathrm{Hg}$. Dans le cas de ${ }^{199} \mathrm{Hg}$, la relaxation est beaucoup plus lente, mais semble due à divers mécanismes dont les effets sont du même ordre de grandeur, et qu'il n'est pas facile de distinguer; cette étude se poursuit actuellement au labcratoire. 


\section{PREMIER CHAPITRE}

LES CONDITIONS DU PONPAGE OPTIQUE.

\section{I - Pompage optique par la raie 2537 요.}

Le spectre du mercure comporte deux raies de résonence situées dans l'ultraviolet; elles relient directement l'état fondanental $6^{1} S_{0}$ aux états excités $6 \mathrm{P}_{1}$ (raie $1850 \AA$ ) et $6^{3} \mathrm{P}_{1}$ (raie $2537 \AA$ ). Le raie $1850^{\circ} \stackrel{\mathrm{A}}{\mathrm{A}}$ est fortenent absorbée par tous les matériaux couramment mployés dans le proche ultraviolet, en particulier en ce qui concerne l'optique de polarisation; elle ne peut au surplus se propager que dans une atmosphère d'azote car elle est absorbée par l'oxygène de l'air, qu'elle transforme en ozone par réaction photochimique. C'est la raison pour lequelle nous avons choisi, comme dens les précédentes tentatives d'utiliser la raie $2537 \AA$.

On peut ici faire une remerque : le temps moyen $T_{p}$ entre l'absorption de deux photons est inverscment proportionnel à la probabilité d'ebsorption d'Einstein, et se trouve être, toutes choses égales d'ailleurs, proportionnel à la durée de vie de l'état excité (14). Or le niveau excité $6^{3} \mathrm{P}_{1}$ de la raie 2537 \& a une durée de vie relativenent longue de $1,2 \cdot 10^{-7}$ seconde (15). Elle est environ 10 fois plus longue que celle de l'état excité de la raie $D$ du sodium, et 100 fois plus longue que celle de l'état excité $6^{1} P_{1}$ de la raie $1850 \AA$ (16). On corprend combien il est indispensable d'obtenir de très fortes intensités lumineuses sur la raie 2537 \& pour que le pornpage optique soit efficace.

On trouvera sur la figure I la structure hyperfine de cette raie d'après les résultats de plusieurs auteurs (17). Nous y avons fait figurer également quelques composantes des deux isomères radioactifs ${ }^{197} \mathrm{Hg}$ de spin $1 / 2$ et $197^{*}$ Hg de spin 13/2. (18). Dans I'état fondamental $6^{l} S_{0}$, le moment cinétique total $F$ est égal au spin 
nucléaire $I$, ce qui conduit pour ${ }^{199} \mathrm{Hg}$ à un niveau $F=I=1 / 2$ et pour ${ }^{201} \mathrm{Hg}$ à un niveau $\mathrm{F}=\mathrm{I}=3 / 2$. Dans I'état excité $6^{3} \mathrm{P}_{1}$ nous avons deux niveaux hyperfins $F=1 / 2$ et $3 / 2$ pour ${ }^{199} \mathrm{Hg}$, et trois niveaux $F=1 / 2,3 / 2$ et $5 / 2$ pour ${ }^{201} \mathrm{Hg}$. C'est pourquoi nous observons dans le spectre de la raie 2537 deux composantes pour ${ }^{199} \mathrm{Hg}$ et trois composantes pour ${ }^{201} \mathrm{Hg}$; nous les désignons dans la suite par la valeur de $F$ du niveau correspondant à l'état excité $6^{3} \mathrm{P}_{1}$. (Rappelons 1 'équivalence avec les notations de Schüler et Keyston : $199-3 / 2=A ; 199-1 / 2=B ; 201-5 / 2=a$; $201-3 / 2=b ; 201-1 / 2=c)$.

Les raies des divers isotopes pairs entre elles sont bien résolues; mais on remarque des coincidences entre certaines raies d'isotopes pairs et certaines composantes des isotopes impairs. La coincidence est presque parfaite entre la composante $199-1 / 2$ et la raie du ${ }^{204} \mathrm{Hg}$, et elle peut être utilisée pour l'excitation sélective de la composante 199 - 1/2 avec une lampe à ${ }^{204} \mathrm{Hg}$. Les composantes $201-5 / 2$ et $201-3 / 2$ sont seulement voisines des raies respectives du ${ }^{204} \mathrm{Hg}$ et du ${ }^{198} \mathrm{Hg}$, à des distances de l'ordre de grandeur de la largeur Doppler. Ces voisinages nous suggèrent $1^{\prime}$ emploi de lampes à isotopes pairs séparés, ${ }^{204} \mathrm{Hg}$ et $198_{\mathrm{Hg}}$, permettant d'obtenir un pompage optique plus efficace sur ${ }^{201} \mathrm{Hg}$ grâce à l'un ou l'autre des deux processus suivants :

a) Si la largeur Doppler de la raie d'émission de la lampe est assez fine, il doit suffire d'appliquer à la lampe un champ magnétique pas trop élevé pour séparer en fréquence les deux composantes $\sigma^{+}$et $\sigma^{-}$et amener une seule d'entre elles à coincider avec la composante voisine de ${ }^{201} \mathrm{Hg}$. Cette méthode de balayage magnétique (décrite au paragrapheIII) permettrait 
d'orienter ${ }^{201} \mathrm{Hg}$ en irradiation $\sigma^{+}$ou $\sigma^{-}$sans utiliser de polariseur, c'est-à-dire avec un gain d'un facteur 10 environ sur l'intensité lumineuse.

b) Si la largeur Doppler de la raie d'émission est au contraire assez large, on doit, sans champ magnétique appliqué à la lampe, exciter avec un bon rendement la composante voisine de ${ }^{201} \mathrm{Hg}$. Cela permettrait d'obtenir un alignement efficace de ${ }^{201} \mathrm{Hg}$ par excitation sélective en lumière naturelle de la composante 201 - 5/2 ou 201 - 3/2, c'est-à-dire en éliminant la composante $201-1 / 2$ qui a une action opposée aux deux autres (elle tend au contraire à vider les sous-niveaux $m_{I}= \pm 3 / 2$ au profit des sous-niveaux $m_{I}= \pm 1 / 2$ (cf paragraphe VI et tableau II). Dans le champ magnétique directeur $\mathrm{H}_{0}$, on observe la décomposition Zeeman des niveaux précédemment cités; les séparations dans I'état fondamental des 2 sous-niveaux $\mathrm{m}_{\mathrm{I}}$ de ${ }^{199} \mathrm{Hg}$ et des 4 sous-niveaux $m_{I}$ de ${ }^{201}{ }^{H g}$ sont dues aux moments magnétiques nucléaires de ces isotopes, et sont très petites par rappcrt aux séparations des sous-niveaux $\mathrm{m}_{\mathrm{F}}$ de 1 : état excité. La décomposition qui en résulte pour la raie 2537 \& est schématisée par les diagrammes de la figure 1 . Les flèches verticales représontent les transitions $\Delta \mathrm{m}_{\mathrm{F}}=0$ en lumière de polarisation $\pi$, rectiligne parallèle à $\mathrm{H}_{0}$; les flèches inclinées vers le haut et la droite représentent les transitions $\Delta \mathrm{m}_{\mathrm{F}}=+1$ en polarisation $\sigma^{+}$, circulaire dans le sens du courant magnétisant; les fJèches inclinées vers le haut et la gauche représentent les transitions $\Delta \mathrm{m}_{\mathrm{F}}=-1$ en polarisation $\sigma^{-}$, circulaire de sens opposé. Les nombres figurant sur les flèches sont les probabilités de transition optique calculées à l'aide des formules de Hill et Inglis (19). 
Ces diagrammes permettent de se représenter de façon plus imagée l'action du pompage optique décrite dans l'introduction : en orientation, l'irradiation $\sigma^{+}$, par exemple, utilise les transitions $\Delta \mathrm{m}_{\mathrm{F}}=+1$ représentées par les flèches inclinées vers le haut et la droite; en alignement, l'irradiation en lumière $\sigma$ Utilise les flèches inclinées dans les deux sens, à l'exclusion des flèches verticales. Les effets de ces deux processus seront calculés en détail dans le paragraphe VI.

\section{II - Les sources Iumineuses.}

Nous avons expliqué au paragraphe précédent l'intérêt qu'il y a à utiliser des lampes à isotopes séparés 204 ou 198. Les échantillons d'isotopes enrichis utilisés étaient d'origine américaine et d'origine française. Ces derniers nous ont été fournis par le service de séparation isotopique du Commissariat à I'Energie Atonique, dont le séparateur à double focalisation (20) permet d'obtenir des taux d'enrichissement élevés, dépassant $90^{\circ} / 0$.

Les faibles quantités dont nous disposions nous ont conduits à porter notre choix sur des lampes sans électrode pour éviter l'absorption du mercure sur les parties métalliques. Il semble en outre que le mercure disparaît a'autant moins rapidement de la décharge, par adsorption sur les parois, que la fréquence utilisée est plus grande (21). Nous avons utilisé comme source H.F. un magnétron continu délivrant 100 Watts à $2400 \mathrm{Mc} / \mathrm{s}$; il est monté dans un générateur commercial (Microtherm de la Raytheon Manufacturing $\mathrm{C}^{\circ}$ ) dont I'antenne est bien adaptée à l'excitation de nos lampes. 
Les lampes sont elles-mêmes en quartz 'Ultrasil": (Heraeus). Elles ont la forme de disques plats de 1 à $2 \mathrm{~mm}$ d'épaisseur intérieure et de 20 à $40 \mathrm{~mm}$ de diamètre. Cette faible épaisseur permet d'éviter l'autoabsorption et le renversement de la partie centrale de la raie $2537 \AA$; elle pernet en outre d'éviter que le volume de vapeur à exciter ne soit trop grand, malgré la grande surface de la lampe. Il est en effet important que la décharge emplisse le volume entier de la lampe pour qu'elle ait un fonctionnement stable; dans le cas contraire, il se produit des oscillations du plasma et la formation de points froids, où le mercure vient se condenser peu à peu, disparaissant ainsi de la décharge.

Les isotopes nous parviennent sous forme d'analgame; il s'agit le plus souvent de la cible en or telle qu'elle est retirée du spectrographe de masse. L'amalgame est chauffé sous vide à $700-800^{\circ} \mathrm{C}$ de façon à faire distiller le mercure dans la lampe, qui est finalement remplie d'argon à une pression de quelques millimètres. Les premières heures de fonctionnenent de la lampe sont toujours délicates quand elle ne contient qu'un milligramme de mercure. On remédie à cet inconvénient par l'opération suivante effectuée sur le banc de pompage avant le remplissage final : étuvage sous vide à $450^{\circ} \mathrm{C}$ puis fonctionnement de la lampe remplie d'argon pur pendant plusieurs heures. In tout état de cause, il est nécessaire de "former"' la lampe dans ses premières heures de fonctionnement; mais ensuite, ce dernier devient très régulier, et une de ces lampes fonctionne encore très bien après deux ans de service intensif (bien que sa brillance ait un peu diminué). Si la lampe devient trop chaude, la raie $2537 \AA$ émise se renverse par auto-absorption et l'intensité de fluorescence observée dans ces conditions baisse considérablement. Il est nécessaire de 
refroidir la lampe sur sa face arrière par un courant d:air, dont on règle le débit de façon à obtenir le signal de résonance optique optimum. Nous travaillons couramment avec une puissance H.F. d'environ 80 Watts et un débit d'air assez fort, parce que ces conditions semblent assurer une meilleure stabilité; mais on peut tirer de la lampe une intensité lurnineuse aussi importante avec une puissance H.F. de 40 Watts seulement et un débit d'air plus faible. Le magnétron étant très sensible aux variations de la tension d'alimentation et de l'impédance de charge H.F'., nous avions adjoint à son alimentation un dispositif stabilisateur de courant. Ce sont cependant les instabilités de l'intensité lumineuse qui ont limité en règle généralo la précision de nos mesures.

\section{III - Les expériences de balayage magnétique.}

Nous avons déjà signalé au paragraphe I l'intérêt qu'il y aurait à pouvoir irradier la vapeur en lumière polarisée circulairement $\sigma^{+}$ou $\sigma^{-}$sans utiliser de polariseurs, parce que nous ne disposons dans l'ultra-violet que de prismes de Glazebrook ou de Nicol, dont l'absorption et la faible ouverture réduisext l'intensité Iunineuse par un facteur d'environ 10. Nous avons essayé à cet usage la méthode de balayage magntitique, employée déjà par d'autres expérimentateurs (22) : elle consiste à exciter les diverises cunpusuites hyperfines de la raic 2537 d li lide de la composante Zeeman $\sigma^{++}$(ou $\sigma^{-}$) diun isotope pair, déplacée en fréquence et séparée de la composante inverse $\sigma^{-}$( ou $\sigma^{+}$), en sournettant à un champ magnétique intense $\mathrm{H}_{\mathrm{S}}$ la source lurnineuse, remplie de cet isotope pair. 
Pour utiliser directement la polarisation circulaire de cette composante déplacée en fréquence, il faut observer la source dans l'axe du champ $\mathrm{H}_{\mathrm{S}}$; ce dernier ne peut donc être produit, si l'on veut un faisceau Iumineux assez ouvert, que par des bobines sans fer. Les seules dont nous disposions permettaient d'obtenir au maximum 1000 Gauss, c'est-à-dire de déplacer les composantes $\sigma^{+}$ou ${\sigma^{-}}^{-}$au maximum de $2100 \mathrm{Mc} / \mathrm{s}$ ou $70.10^{-3} \mathrm{~cm}^{-1}$. Nos essais de balayage magnétique se trouvaient donc limités aux couples de composantes voisines déjà signalés : 204 et $201-5 / 2$, ou 198 et 201 - 3/2. Leurs intervalles ne sont pas connus avec une grande précision, mais seraient de l'ordre de $800 \mathrm{Mc} / \mathrm{s}$ et $650 \mathrm{Mc} / \mathrm{s}$ respectivement. La largeur Doppler calculée pour la raie $2537 \AA$ dans une vapeur à température ordinaire est de $1000 \mathrm{Mc} / \mathrm{s}$; et si $1 \mathrm{a}$ source lumineuse n'émet pas une raie beaucoup plus large, il est possible que l'on puisse séparer suffisarment les composantes

$$
\begin{aligned}
& \sigma^{+} \text {et } \sigma^{-} \text {. } \\
& \quad \text { Pour tester le procédé, nous avons utilisé le montage }
\end{aligned}
$$
schématisé sur la figure 2. La source à ${ }^{204} \mathrm{Hg}$ ou ${ }^{198}$ Hg est placée au centre des bobines fournissant le champ $\mathrm{H}_{\mathrm{s}}$; elle irradie parallèlement à $\mathrm{H}_{\mathrm{S}}$ une cellule contenant la vapeur de ${ }^{201} \mathrm{Hg}$, soumise à un faible champ $\mathrm{H}_{0}$ parallèle à $\mathrm{H}_{\mathrm{s}}$. Un analyseur circulaire (lame quart d'onde suivie d'un Glazebrook) est placé sur le trajet du faisceau d'irradiation; il ne laisse passer que la composante $\sigma^{+}$(ou la composante $\sigma^{\circ}$ ) émise par la lampe, dont la fréquence est augmentée (ou diminuée) proportionnellement à $\mathrm{H}_{\mathrm{s}}$. On mesure avec un photomultiplicateur la lumière de résonance optique $I\left(\mathrm{H}_{\mathrm{S}}\right)$ réémise par la vapeur à angle droit de l'irradiation. On obtiendra le signal maximum quand la composante sélectionnée à l'irradiation coïncidera en fréquence avec la composante voisine du ${ }^{201} \mathrm{Hg}$. 
(Remarque : La polarisation de la lumière est transformée par l'analyseur et la cellule reçoit de toute façon de la lumière polarisée rectiligne qui est absorbée par la vapeur de ${ }^{201} \mathrm{Hg}$ comme un mélange à parts égales des deux polarisations $\sigma^{+}$et $\sigma^{-}$; mais comme les probabilités de transition optique sont symétriques en $\sigma^{+}$et en $\boldsymbol{\sigma}^{-}$, l'absorption n'est pas modifiée par ce changement de polarisation).

La figure 3 donne les résultats obtenus respectivement avec une lampe à ${ }^{204} \mathrm{Hg}$ et une lampe à ${ }^{198} \mathrm{Hg}$. En ordonnée sont portées les variations relatives de l'intensité réémise, normée à la valeur 1 en champ $\mathrm{H}_{\mathrm{S}}$ nuI : $\mathrm{I}\left(\mathrm{H}_{\mathrm{s}}\right) / \mathrm{I}(\mathrm{O})$. En abscisse, le champ $\mathrm{H}_{\mathrm{S}}$ est porté vers la gauche lorsque l'analyseur sélectionne la composante $\sigma^{+}$de la lampe; il est porté vers la droite lorsque l'analyseur sélectionne la composante $\sigma^{-}$de la lampe (après rotation de $90^{\circ}$ de la lame quart d'onde). Les deux courbes se raccordent en champ nul et donnent une image de la forme de la raie émise par la lampe sur l'échelle des fréquences. (Pour une interprétation exacte, voir annexe I).

Dans chaque cas, sont représentées deux courbes correspondant à des régimes de fonctionnement différents de la lampe. La courbe la plus étroite correspond au refroidissement maximum de la lampe (au-delà la décharge devient instable), lorsque l'intensité I (0) émise est faible. La courbe la plus large correspond à un refroidissement moindre; l'intensité I (0) est dans ce cas deux fois plus élevée, mais elle reste encore très inférieure au maximum d'intensité que l'on peut obtenir en réglant convenahiement le refroidissement de la lampe. La précision des mesures est limitée par deux effets : a) I'échantillon de ${ }^{201} \mathrm{Hg}$ utilisé lors de cette expérience n'était enrichi qu'à $70 \%$ et contenait encore $1 \%$ de ${ }^{204} \mathrm{Hg}, 1,5 \%$ de ${ }^{198} \mathrm{Hg}$ et $3 \%$ de ${ }^{199} \mathrm{Hg}$, ce qui peut déformer légèrement la courbe tracée. b) le rérime de décharge dans la lampe est sensible au 
champ $\mathrm{H}_{\mathrm{s}}$; et les corrections effectuées en conséquence laissent une certaine marge d'erreur.

Voici les conclusions de ces mesures :

- a) Les séparations des composantes 204 et 201 - 5/2 d'une part, 198 et $201-3 / 2$ a'autre part sont presque égales et de l'ordre de $550 \mathrm{Mc} / \mathrm{s}$ ou $18.10^{-3} \mathrm{~cm}^{-1}$, un peu inférieures aux valeurs généralement admises (17).

- b) La largeur Doppler de la raie d'érission vaut au minimum $1600 \mathrm{Mc} / \mathrm{s}$ (cf annexe I) dans les conditions de refroidissement maximum, où la lampe est peu brillante. Elle est très supérieure à cette valeur dans les conditions de refroidissement qui assurent le maximum d'intensité de le lampe.

- c) L'excitation sélective dos composantes 201 - 5/2 ou 201 - 3/2 par des lampes à ${ }^{204} \mathrm{Hg}$ ou ${ }^{198} \mathrm{Hg}$ est toujours possible avec un excellent rendement en champ $H_{s}$ nul.

- d) La séparation magnétique des composantes $\sigma^{+}$et $\sigma^{-}$ sans dispositif de polarisation n'est pas utilisable en pratique. Ia séparation $n^{i e s t ~ e n ~ e f f e t ~ p o s s i b l e ~ q u ' a v e c ~ u n e ~ l a m p e ~ t r e ̀ s ~ r e f r o i d i e, ~}$ c'est-à-dire au prix d'une perte considérable sur I'intensité lumineuse de la composante utile. Et même dans ce cas, à l'optimum de

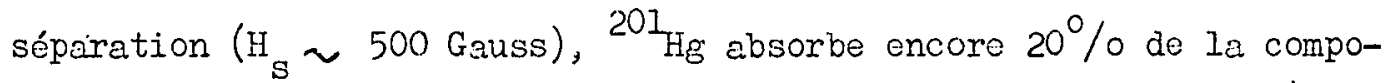
sante circulaire à éliminer (Le mélange des deux conposantes $\sigma^{+}$et $\sigma^{-}$en quantités inégales rend très délicate l'interprétation précise des courbes de résonance magnétique observées). Nous avons donc renoncé à utiliser le balayage magnétique dans nos études ultérieures sur ${ }^{201} \mathrm{Hg}$.

- e) Cette méthode de séparation doit au contraire se révéler favorable pour orienter les deux iscrieres radioactifs ${ }^{197} \mathrm{Hg}$ et $197^{\star} \mathrm{Hg}$, en excitant les composantes $197-1 / 2$ et $197^{*}-15 / 2$ avec une lampe à ${ }^{204} \mathrm{Hg}$ soumise à un champ $\mathrm{H}_{\mathrm{s}}$ de 1000 à 1500 Gauss. 


\section{IV - Le montage expérimental.}

La figure 4 montre le schéma du montage utilisé pour réaliser le pompage optique. Le charnp $\mathrm{H}_{0}$ appliqué à la vapeur étudiée est produit par deux bobines de $72 \mathrm{~cm}$ de diamètre moyen placées en position d'Helmholtz. Leur écartement et leur parallélisne sont réglés à l'aide de quatre tiges filetées sur lesquelles elles sont fixées rigidement par des écrous. Ayant réglé le parallélisme des bobines, on peut déplacer une des bobines dans son plan, horizontalement et jerticalement, de manière à amener leurs axes à coincider exactement. Ces réglages sont effectuée par approximations successives en contrôlant la valeur du champ magnétique en différents points autour du centre à l'aide d'une résonance de protons; ils permettent d'obtenir une excellente homogénéité (cf paragraphe VIII).

Chaque bobine contient environ 8000 tours et a une résistance un peu supérieure à 500 Ohms. Elles sont alimentées en série par une alimentation à haute stabilité Varian. Le champ produit au centre du système est d'environ 210 Gauss/Ampère, et peut monter à près de 400 Gauss Iorsquion utilise la puissance maximum de I'Elimentation (2000 V, 2 Ampères). Les bobines sont orientées de façon que leur axe coincide avec la direction de la composante horizontale du champ terrestre; nous avons toujours utilisé des champs $\mathrm{H}_{0}$ assez forts pour qu'il ne soit pas utile de compenser la composante verticale du champ Lerrestite.

A l'intérieur de ce système de bobines principal se trouvent encore une paire de bobines de même axe, produisant le champ de balayage alternatif nécessaire à l'observation de la résonance de protons (cf paragraphe VIII), et une paire de bobines d'axe vertical, servant à produire le champ de radiofréquence $H_{1}$ (cf paragraphe IX). 
Au centre géométrique des bobines est placée la cellule contenant la vapeur de l'isotope étudié. Les cellules sont en quartz "Ultrasil'" (Heraeus) et sont de forme cubique; nous utilisons deux dimensions : $2 \mathrm{~cm}$ et $4 \mathrm{~cm}$ d'arête. Au centre de I'une des six faces est soudé le queusot, tube mince d'environ $30 \mathrm{~cm}$ de long, dont l'extrémité est maintenue à température fixe $t\left(-30^{\circ} \mathrm{C}\right.$ à $\left.0^{\circ} \mathrm{C}\right)$, et où se condense la goutte de mercure. Le thermostat est constitué par un vase Dewar traversé par un courant d'air refroidi par barbottage préalable dans l'air liquide; la soupape d'arrivée de cet air est commandée par un thermomètre à contact, par l'intermédiaire d'un relais à thyratron. Nous donnons dans le tableau ci-dessous les valeurs calculées (23) du nombre d'atomes de mercure par $\mathrm{cm}^{3}, \mathrm{~N}$, dans une cellule à température ordinaire en équilibre avec la goutte liquide à température $t$ dans le queusot.

\begin{tabular}{|c|c|c|c|c|c|c|c|c|c|c|}
\hline en ${ }^{\circ} \mathrm{C}$ & $:-35^{\circ}$ & $-30^{\circ}$ & $-25^{\circ}$ & $-20^{\circ}$ & $-15^{\circ}$ & $-10^{\circ}$ & $-5^{\circ}$ & $0^{\circ}$ & $+5^{\circ}$ & $+10^{\circ}$ \\
\hline $\begin{array}{l}J \text { en } 10^{+12} \\
\text { atomes } / \mathrm{cm}^{3}\end{array}$ & 0,08 & 0,17 & 0,34 & 0,63 & 1,16 & 2,09 & 3,60 & 6,25 & 10,0 & 16,3 \\
\hline
\end{tabular}

I'irradiation lumineuse se fait suivant l'axe du champ $H_{0}$. L'image de la lampe est formée sur la cellule à l'aide de deux lentilles en silice fondue de grande ouverture (focale $20 \mathrm{~cm}$, diamètre $12 \mathrm{~cm}$ ). On peut ainsi concentrer sur la cellule un flux lumineux important, pour aligner ${ }^{201} \mathrm{Hg}$. Si l'on cherche à produire une orientation nucléaire, il faut interposer sur le faisceau d'irradiation un prisme polariseur (Glazebrook collé à la glycérine, ou Nicol collé au glucose cristal) suivi d'une lame quart d'onde (lame $(2 n+1) \lambda / 4$ en quartz, taillée par Madame Devaux-Morin). La réduction d'intensité lumineuse qui en résulte, par absorption et réduction de l'ouverture du faisceau, atteint un facteur d'environ 10. 
La détection de l'orientation ou de l'alignement peut se faire en mesurant les variations de l'intensité lumineuse transmise à travers la cellule. Mais nous n'avons pas utilisé cette méthode pour les deux raisons suivantes : a) La largour de la raie ćmise par la lampe excède de beaucoup la largeur Doppler d:absorption dans la cellule (cf paragraphe III); et il est pratiquement inpossible d'éliminer le groupe très intense des raies du nercure au voisinage de $3600 \AA-b)$ Pour observer une absorption notable, il faut admettre dans la cellule une densité de vapeur relativenent forte, ce qui oblige à tenir compte de la loi exponentielle d'absorption. Et les signaux observés ne sont plus linéaires par rapport aux populations des sous-niveaux Zeeman, si leurs variations sont importantes. Notons en outre que la diffusion multiple (15) doit jouer aux fortes densités de vapeur un rôle appréciable dont I'étude reste à faire.

Nous avons donc choisi de travailler à faible densité de la vapeur de mercure dans la cellule de résonance (queusot à $t=-20^{\circ} \mathrm{C}$ ou $-30^{\circ} \mathrm{C}$ ), et d'utiliser pour la détection la lumière de résonance optique rámise par la vapeur à angle droit du faisceau d'irradiation. On mesure les variations soit de son intensité soit de son degré de polarisation lorsqu'une résonance nagnétique à saturation rétablit entre les sous-niveaux Zeeman de l'état fondamental l'égalité des populations. Dens le cas de l'excitation sélective de la composante hyperfine $199-1 / 2$ en polarisation $\sigma^{+}$par une lampe à ${ }^{204} \mathrm{Hg}$ par exemple, on ne met en jeu qu'un seul sous-niveau de l'état excité et la lunière réénise est toujours complètenent dépolarisée; la détection se fait on mesurant avec un photomultiplicateur l'intensité de la lumière rćénise, qui est proportionnelle à la population du sous-niveau $\mathrm{m}_{I}=-1 / 2$. Cette méthode de détection est 
simple, mais a l'inconvénient d'être très sensible aux fluctuations de la source lumineuse.

Dans les études sur ${ }^{201} \mathrm{Hg}$ excité perr des larpes à ${ }^{204} \mathrm{Hg}$ ou ${ }^{198} \mathrm{Hg}$, nous aurions pu utiliser également cette simple mesure d'intensité. Mais il est préférable de mesurer le degré de polarisation de la lumière réémise par la méthode différentielle classique (3)(4), qui permet d'atténuer les effets des fluctuations de la lampe. Pour cette mesure on utilise deux bancs d'optique identiques perpendiculaires à l'axe d'irradiation de part et d'autre de la cellule : une lentille en silice fondue (non dépolarisante) forme l'image de la cellule sur la cathode d'un photomultiplicateur RCA IP28, à travers un prisme de Glazebrook. Les deux Glazebrook, de part et d'autre, sont orientés de façon à isoler l'un la vibration $\pi$ parallèle à $\mathrm{H}_{0}$, I'autre la vibration $\sigma^{-}$perpendiculaire à $\mathrm{H}_{0}$. Les signaux $I_{\pi}$ et $I_{\sigma}$ mesurés par les deux photomultiplicateurs sont opposés dans un pont de résistances schématisé sur la figure 4. Si le pont est équilibré pour un certain état des populations dénoté par l'indice zéro, le courant mesuré ensuite dans le galvanomètre est proportionnel à la quantité :

$$
\text { (IV-I) } P=\frac{I_{\pi}}{I_{\pi_{0}}}-\frac{I_{\sigma^{N}}}{I_{\sigma_{0}}}=\frac{\Delta I_{\pi}}{I_{\pi_{0}}}-\frac{\Delta I_{\sigma}}{I_{\sigma_{0}}}
$$

$V$ - Relation entre les signaux détectés et les populations.

Les signaux fournis par notre méthode de détection (variations de l'intensité ou du degré de polarisation $P$.) sont en relation avec les variations des populations $X_{m}$ des sous-niveaux Zeernen $m$ de 
l'état fondamental sous l'action de la résonance magnétique. Il est important de connaître exactement cette relation pour vérifier l'ampleur de ces effets. Cette relation nous permettra à partir des signaux observés de calculer les variations des populations qui se produisent, lorsque la résonance magnétique à saturation égalise les populations d'équilibre atteintes par le pompage optique.

Cette relation est également nécessaire, en général pour calculer la forme des signaux de résononce magnétique observés à partir des calculs d'évolution des populations sous l'action du champ de radiofréquence (analogues à ceux de la fin du troisième chapitre). Mais ce problème ne se pose pour nous que dans lo cas de l'orientation de ${ }^{201} \mathrm{Hg}$. En effet, dans le cas de I'alignoment de ${ }^{201} \mathrm{Hg}$ (grâce à la symétrie entre les sous-niveaux de même valeur absolue ( $\mathrm{m} \mathrm{I}$ ), et dans le cas de ${ }^{199} \mathrm{Hg}$ les populations ne dépendent que d'une seule variable indépendante; et, si nous travaillons à des densités de vapeur assez faibles, nous savons que les signaux observés sont des fonctions linéaires des populations; les variations des signaux sont donc dans ces deux cas, proportionnelles aux variations de cette unique variable.

Le calcul se fait à partir des probabilités de transition optique. Nous appelons $\mathrm{A}_{\mathrm{m}}^{\mathrm{F}, \mathrm{m}^{\prime}}$ celle qui correspond à la transition entre le sous-niveau in de l'état fondamental et le sous-niveau $\left(F, \mathrm{~m}^{\prime}\right)$ de $\mathrm{l}^{\prime}$ état excité. Nous les supposons normées en sorte que $\sum_{\mathrm{F}} \mathrm{A}_{\mathrm{m}} \mathrm{F}^{\prime} \mathrm{m}^{\prime}=\sum_{\mathrm{m}} \mathrm{A}_{\mathrm{m}} \mathrm{F}, \mathrm{m}^{\prime}=1$, c'est-à-dire qu'on les obtient en divisant par 30 les nombres indiqués sur les schémas de la figure 1. Soit $\Phi(\nu) . d \nu$ le nombre de photons par $\mathrm{cm}^{2}$ et par seconde arrivant sur la cellule avec la polarisation $\sigma^{+}$et dans la bande de fréquence comprise entre $\nu$ et $\nu+d \nu$. Dans les conditions normales d'utilisation, nos lampes émettent des raies beaucoup plus larges 
que la raie d'absorption dans la cellule (cf paragraphe III); nous pouvons donc supposer $\Phi(\nu)$ constant sur toute la bande de fréquence où une composante hyperfine donnée présente une absorption notable. Si de plus, le nombre d'atomes de mercure par $\mathrm{cm}^{3} \mathrm{~N}$ est suffisamment faible (pour que la loi exponentielle d'absorption soit assimilable à une loi linéaire), on démontre à partir de la théorie du rayonnement d'Einstein (14) que le nombre de photons absorbés en 1 seconde par $1 \mathrm{~cm}^{3}$ de vapeur dans la transition $\mathrm{m} \rightarrow\left(\mathrm{F}, \mathrm{m}^{\prime}=\mathrm{m}+1\right)$ est :

(V-2) $\quad \frac{3 \lambda^{2}}{8 \pi \tau} \Phi \mathbb{N}_{m} A_{m}^{F, m+1}=\frac{3 \lambda^{2}}{8 \pi \tau} \Phi-\frac{N}{2 I+1} X_{m} A_{m}^{F, m+1}$

$\lambda$ est la longueur d'onde de la raie et $\tau$ la durée de vie de l'état excité (15); le facteur 3 représente le rapport $g_{2} / g_{1}$ des poids statistiques de I'état excité et de l'état fondamental pour la raie $2537 \AA$. $\mathrm{N}_{\mathrm{m}}$ est le nombre par $\mathrm{cm}^{3}$ d'atomes occupant le sous-niveau $\mathrm{m}$; nous appelons populations de ces sous-niveaux les quantités $X_{m}$ proportionnelles aux nombres $\mathbb{N}_{m}$, mais indépendantes de la densité de vapeur $\mathbb{N}$, et qui vérifient $I$ 'équation de normalisation $\sum_{m} X_{m}=2 I+1$; c'est-à-dire qu'à I:équilibre thermique $\mathrm{X}_{\mathrm{m}}=1$ pour tout $\mathrm{m}$. (Rappelons que les différences d"énergie Zeernan sont négligeables devant $\mathrm{kT}$ ).

La formule (V-2) nous donne le nombre d'atomes qui ont été portés au sous-niveau $(F, m+l)$ de l'état excité par seconde et par $\mathrm{cm}^{3}$ de vapeur. Ces atomes sont retombés à l'état fondamental en émettant de la lumière $\sigma^{+}, \pi$ et $\sigma^{-}$proportionnellement aux quantités $A_{m}^{F, m+I}, A_{m+1}^{F, m+l}$ et $A_{m+2}^{F, m+1}$. En faisant la somme des contributions de tous les sous-niveaux $(F, m+l)$ de l'état excité hyperfin $F$, on obtient les nombres de photons réémis en 1 seconde par $1 \mathrm{~cm}^{3}$ de vapeur dans les diverses polarisations (rappelons que nous supposons $1^{\prime}$ irradiation en $\sigma^{+}$) : 


$$
I_{\pi}=\frac{3 \lambda^{2}}{8 \pi \tau} \Phi \frac{N}{2 I+1} \sum_{m} X_{m} \cdot A_{m}^{F, m+1} \cdot A_{m+1}^{F, m+1}
$$

$(\mathrm{V}-3)$

$$
I_{\sigma}=\frac{I_{\sigma^{+}+} I_{\sigma^{-}}}{2}=\frac{3 \lambda^{2}}{8 \pi \tau} \Phi \frac{N}{2 I+1} \sum_{m} X_{m} \cdot A_{\mathrm{rn}}^{F, m+1} \frac{A_{m}^{F, m+1}+A_{m+2}^{F, m+1}}{2}
$$

Dans le cas d'une irradiation du type 'broad linel', c'est-à-dire d'une excitation simultanée des diverses composantes hyperfines avec la même intensité $\Phi$, il suffit d'étendre la sommation de ces formules aux diverses valeurs de $F$. Dans le cas de l'irradiation $\sigma$, en lumière naturelle dans l'axe du champ $\mathrm{H}_{0}$, il faut ajouter aux termes donnés en ( $\mathrm{V}-3$ ) les contributions calculées de manière symétrique pour l'irradiation en $\sigma^{-}$.

Les résultats de ces calculs sont donnés à la fin de ce chapitre, dans les tableaux I et II (colonnes 5 à 9) pour les deux cas d'orientation en $\sigma^{+}$de ${ }^{199} \mathrm{Hg}$ et d'alignement de ${ }^{201_{\mathrm{Hg}}}$, et dans le tableau III (colonnes 4 et 5) pour le cas d'orientation en $\sigma^{+}$de ${ }^{201} \mathrm{Hg}$. A I'intérieur de chacun de ces trois cas, nous calculons séparément, à $\Phi$ constant, I'excitation sélective de chaque composante hyperfine et I'excitation "broad line"'; les intensités $I_{\pi}$ et $I_{\sigma}$ sont données à un facteur de proportionnalité près, qui est le même à l'intérieur de chaque cas. Pour alléger la présentation, nous adoptons pour les populations les notations indiquées sur la figure 1 et qui nous servirons dans los calculs du troisième chapitre :

$$
\begin{aligned}
& \text { pour }{ }^{199} \mathrm{Hg} \quad x_{+1 / 2}=\alpha \quad x_{-1 / 2}=\beta \\
& \text { pour }{ }^{201} \mathrm{Hg} \quad x_{+3 / 2}=\alpha \quad x_{+1 / 2}=\beta \quad x_{-1 / 2}=\gamma \quad x_{-3 / 2}=\eta
\end{aligned}
$$

Dans le cas d'alignement de ${ }^{20 l_{H g}}$, la symétrie entre les sousnivcaux $\pm \mathrm{m}$ permet de calculer $I_{\pi}$ et $I_{\sigma}$ (colonnes 5 et 6 du tableau II) en fonction de 2 variables seulement $\alpha=\gamma$ ) et $\beta=\gamma$. Nous donnons également dans les colonnes 7 et 8 des tableaux I et II les variations relatives de $I_{\pi}$ et $I_{\sigma}$ en fonction des variations des populations qui seront provo- 
quées par la résonance magnétique : dans le cas d'orientation de ${ }^{199}{ } \mathrm{Hg}$ (tableau I) $\Delta \beta=-\Delta \alpha$; dans le cas d'alignement de ${ }^{201} \mathrm{Hg}$ (tableau II) $\Delta \beta=\Delta \gamma=-\Delta \alpha=-\Delta \eta$.

Pour simplifier le calcul de ces variations, et celui du signal de polarisation $P=\Delta I_{\pi} / I_{\pi_{0}}-\Delta I_{\sigma} / I_{\sigma}$ (colonne 9 des tableaux $I$ et II; colonne 5 du tableau III), nous supposons que l'équilibre du pont (état 0) est réglé à l'équilibre thermique de la vapeur (populations toutes égales). Ceci n'est pas vrai dans la pratique puisque on général nous équilibrons le pont de résistances sur la vapeur orientée et observons le déséquilibre provoqué par la résonance magnétique; mais tant que les variations des populations restent faibles, ce calcul constitue une très bonne approximation.

On vérifie sur ces tableaux que la mesure du taux de polarisation ne peut jamais servir à la détection lorsqu' on excite sélectivement un état excité hyperfin $F=1 / 2$. On voit aussi que, dans le cas du ${ }^{201} \mathrm{Hg}$, une lampe à ${ }^{204} \mathrm{Hg}$ excitant sélectivement le niveau 5,'2 doit fournir tant en orientation qu'en alignement les signaux les plus importants pour la mêne variation des populations.

VI - Evaluation de l'efficacité du nompage optique.

Nous nous proposons, dans ce paragraphe, d'analyser en détail l'action du pompage optique pour comparer son efficacité dans les différents cas d'excitation optique. $\Lambda$ cet effet, nous voulons calculer ici, indépenáamment de tout caicuI de résonance magnétique, Ies populations d'équilibre qu'il permet d'atteindre. Nous avons déjà dit dans l'introduction que ces populations d'équilibre résultent de la compétition entre le pompage optique et la relaxation désorientante; elles dépendent du rapport $T_{I} / T_{p}$ du temps de relaxation zongitudinale $T_{I}$ au temps moyen $T_{p}$ entre l'absorption de deux photons successifs par le même 
atome. Si nous calculons les populations en fonction de ce rapport $\mathrm{T}_{1} / \mathrm{T}_{\mathrm{p}}$, nous pourrons inversement calculcr le rapport à partir des signaux observés.

Ce temps $T_{p}$ nécessite une définition plus précise. En effet, dans le cas où l'on excite les atomes sélectivement avec une seule composante hyperfine, la probabilité $1 / T_{m}$ par unité de temps pour que les atomes du sous-niveau $\mathrm{m}$ de l'état fondarnental absorbent un photon dépend de $\mathrm{m}$. Nous introduisons alors $\mathrm{I} / \mathrm{T}_{\mathrm{p}}$ comme la moyenne de ces probabilités :

(VI-4) $\quad \frac{1}{T_{p}}=\frac{1}{2 I+1} \sum_{m} \frac{1}{T_{m}}$

On obtient $1 / T_{m}$ en divisant par le nombre d'atomes absorbants $\mathrm{N}_{\mathrm{m}}$ le nombre de photons absorbés par seconde dans $1 \mathrm{~cm}^{3}$ de vapeur, soit $d^{\prime}$ après (V-2) dans le cas d'une irradiation en $\mathrm{G}^{+}$avec une seule composante hyperfine :

(VI-5) $\frac{1}{T_{m}}=\frac{3 \lambda^{2}}{8 \pi \tau} \Phi A_{m}^{F, m+1}$ di où $\frac{1}{T_{p}}=\frac{3 \lambda^{2}}{8 \pi \tau} \Phi \frac{\sum_{m} A_{m}^{F, m+1}}{2 I+I}$

On en déduit facilement la formule valable dans les cas d'irradiation avec la polarisation $\sigma^{-}$ou avec les deux polarisations simultanées $\sigma^{+}$et $\sigma^{-}$. Dans le cas de l'excitation " broad line " , il faut dans (VI-5) faire la somne $\sum_{\mathrm{F}} \mathrm{A}_{\mathrm{m}} \mathrm{F}, \mathrm{m+1}$ qui est égale à 1 , en sorte que $\mathrm{T}_{\mathrm{m}}$ est, dans ce cas, indépendant de $\mathrm{m}$ :

(VI-6) $\quad \frac{1}{T_{m} !}=\frac{1}{T_{p}^{\prime} !}=\frac{3 \lambda^{2}}{3 \pi \tau} \Phi \quad$ (en ''broad line'")

La probabilité $1 / T_{p}$ ainsi définie est le paramètre important dans le calcul de la résonance magnétique (cf troisième chapitre); mais elle prósente l'inconvénient de ne pas représenter de façon univoque l'intensité lunineuse $\Phi$, puisque le coefficient de proportionnalité dépend de la composante hyperfine utilisée. Dans le présent calcul, s'introduit de 
manière beaucoup plus naturelle, à la place du paramètre $T_{1} / T_{p}$, un autre paramètre qui représente mieux l'intensité lumineuse :

$$
\text { (VI-7) } r=\frac{3 \lambda^{2}}{8 \pi \tau} \Phi \cdot \mathrm{T}_{1} \begin{cases}=\frac{\mathrm{T}_{1}}{\mathrm{~T}_{\mathrm{p}}^{\prime}} & \text { en excitation "broad line". } \\
=\frac{\mathrm{T}_{1}}{\mathrm{~T}_{\mathrm{p}}} \cdot \frac{2 \mathrm{I}+1}{\mathrm{~F}_{\mathrm{m}} \mathrm{F}, \mathrm{m}+1} & \begin{array}{l}
\text { en excitation par une } \\
\text { componte séparée. }
\end{array}\end{cases}
$$

Pour étudier l'action simultanée du pompage et de la relaxation, nous ne pouvons pas utiliser exactement le formalisme introduit par Cohon-Tannoudji (24), du fait que $\mathrm{T}_{\mathrm{m}}$ varic avec $\mathrm{m}$. Pour la relaxation, nous supposons commo lui que toutes les populations tendent vers 1 avec la même constante de ternps $\mathrm{T}_{I}$. Pour le pompage optique, nous faisons le bilan des atomes arrachés à l'état fondamental par les photons orientateurs et des atomes retorabant do l'état excité dans les sous-nj.veaux de l'état fondamental. La durée de vie $\tau$ de l'état excité étant bealucoup plus courte que $T_{p}$, nous pouvons écrire dans le cas d'irradiation en $\sigma^{+}$par une seule composante hyperfine :

(VI-8) $\frac{d X_{m}}{d t}=\frac{1-X_{n}}{T_{1}}-\frac{X_{m}}{T_{m}} \div \sum_{n} \frac{3 \lambda^{2}}{8 \pi \tau} \Phi \cdot X_{n} \cdot A_{n}^{F, n+1} \cdot A_{m}^{F, n+1}$

(seuls les temes $n=m, m+l$ et $m+2$ apportent une contribution à la some $\sum$ ). Dans le cas de l'excitation "broad line" on remplacerait $T_{m}$ par $T_{m}^{\prime}=T_{p}^{p}$ et on effectuerait dans le teme de droite la somation sur F en plus de la sormation $\operatorname{sur} n$. Nous cherchons la solution stationnaire de ce système différentiel, ce qui nous conduit au systène d'équations linéaires :

(VI-9) $0=1-X_{m}+r\left[-A_{m}^{F, m+1} \cdot X_{m}+\sum_{n} X_{n} \cdot A_{n}^{F, n+1} \cdot A_{m}^{F, n+1}\right]$ 
Ce sont les solutions de ce système que nous donnons dans les tableaux I, II (colonnes 2, 3 et 4) et dans le tableau III (colonnes 2 et 3). Les tableaux I et II donnent les résultats dans les cas d'orientation du ${ }^{199} 9_{\mathrm{Hg}}$ et d'alignement du ${ }^{201} \mathrm{Hg}$; nous avons déjà remarqué que dans ces deux cas, les populations ne dépendent que d'une seule variable indépendante, ce qui rend le calcul particulièrement simple. Nous donnons dans une première colonne la valeur de $r$ en fonction de $\mathbb{T}_{1} / T_{p}$, dans la deuxième colonne, la valeur exacte en fonction de $r$ des quantités $\alpha-1=1-\beta$ pour $199_{\mathrm{Hg}}$, et des quantités $\alpha-1=1-\beta=\eta-1=1-\gamma$ dans le cas de I'alignement de ${ }^{201} \mathrm{Hg}$; dans les deux colonnes suivantes, nous donnons les valeurs limites vers lesquelles tendent $\alpha$ et $\beta$ aux fortes intensités lumineuses quand $r$ devient infini. On voit que la composante $201-1 / 2$, utilisée seule, pompo aussi efficacement les atomes que la composante $201-5 / 2$, mais en sens inverse; et on mesure l'intérêt des lampes à isotopes séparés pour réaliser l'alignenent de ${ }^{201} \mathrm{Hg}$. Le tableau III correspond à l'orientation du ${ }^{201} \mathrm{Hg}$ en irradiation $\sigma^{+}$; les calculs sont. plus compliqués et nous ne donnons pas les eojutions exactes, mais seulement les valeurs approchées quand $r$ est faible (colonne 2) et les valeurs limites quand $r$ devient infini (colonne 3). Dans la comparaison des ta-

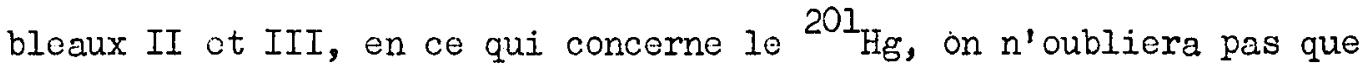
nous avons en alignement des intensités lumineuses 10 fois plus grandes, c'est-à-dire un paramètre $r 10$ fois plus grand que dans le cas de I'orientation. La comparaison directe entre le cas du ${ }^{199} \mathrm{Hg}$ (tableau I) et le cas du ${ }^{201} \mathrm{Hg}$ (tableaux II et III) n'est pas possible car leurs temps de relaxation 'l' sont très différents. 


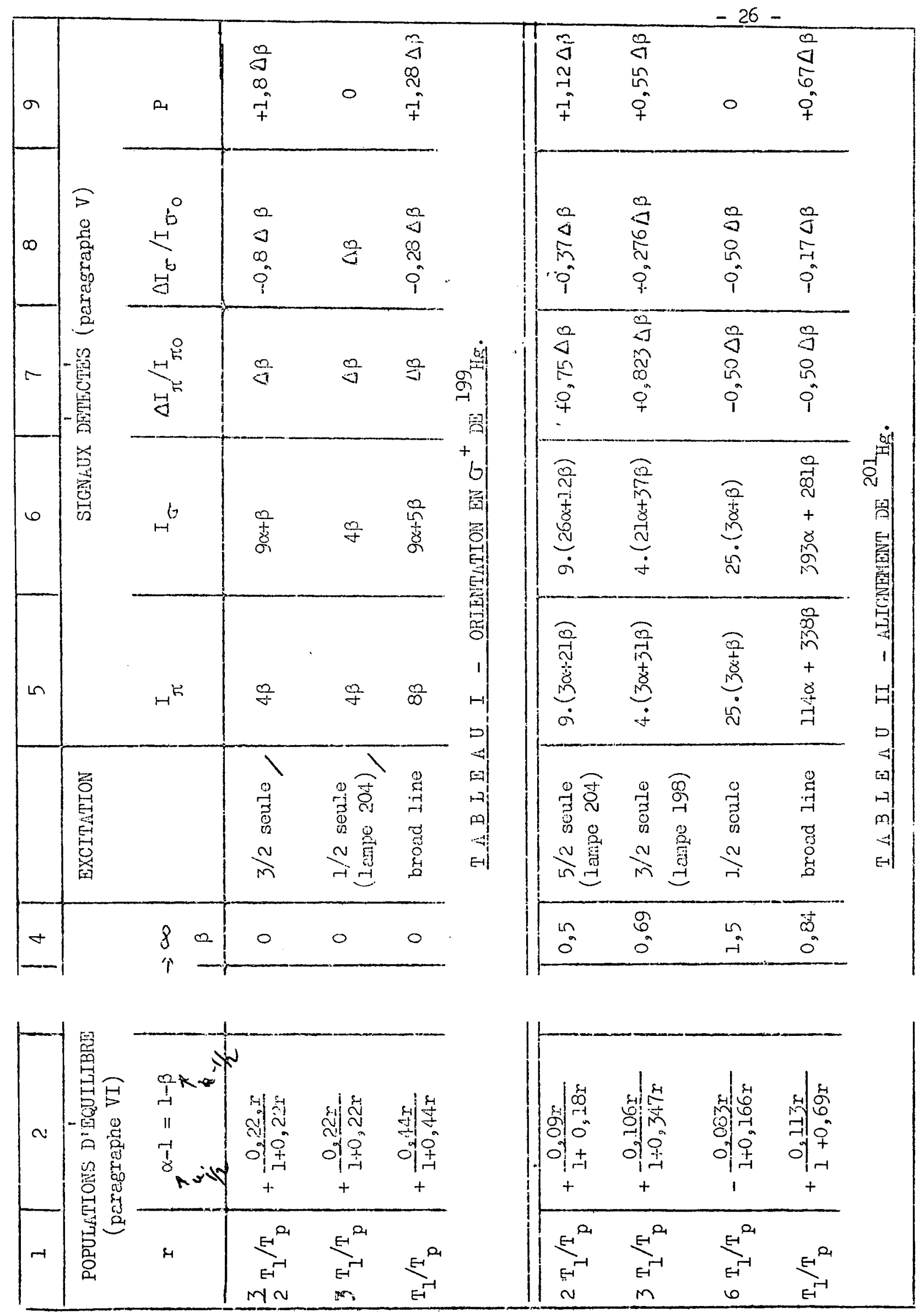




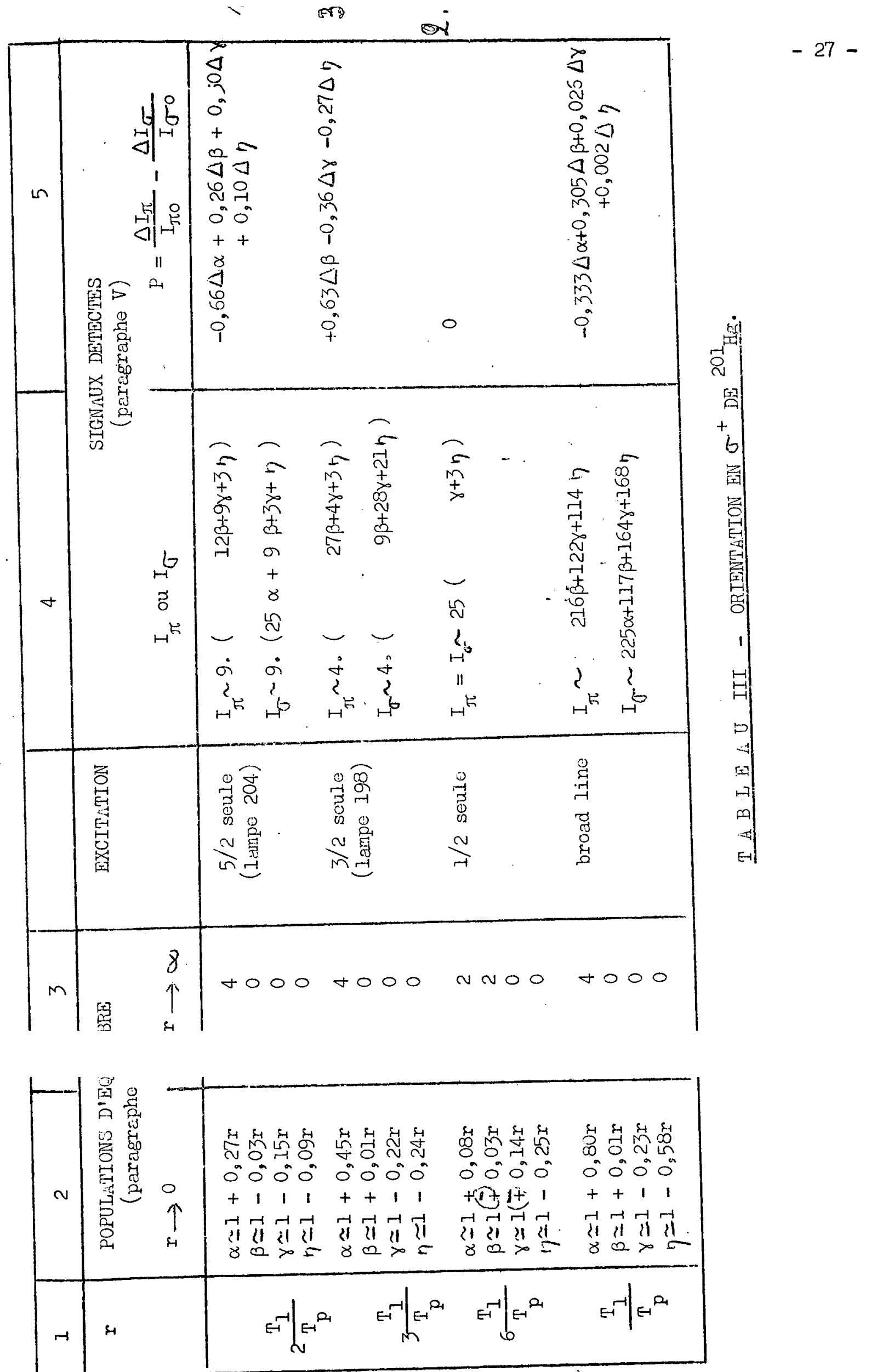




\section{DEUXIEME CHEPITRE} MESUFE DES MONENTS NUCLEATRES.

\section{VII - Mesures préliminaires à fréquence fixe.}

La possibilité d'obtenir en alignement des intensités lumineuses pompantes beaucoup plus importantes qu'en orientation nous a conduit à tenter nos premières expériences dans le cas de 1' alignement de ${ }^{201}$ Hg. Nous avons cherché à observer les résonances magnétiques entre les sous-niveaux m de l'état fondainental par la méthode classique en balayant le charmp directeur $\mathrm{H}_{\mathrm{O}}$, à fréquence fixe du champ de radiofréquence $\mathrm{H}_{1}$. Un système à démultiplication de fréquence, à partir soit d'un quartz à $100 \mathrm{kc} / \mathrm{s}$, soit de la fréquence porteuse du poste de radiodiffusion de Droitwich $(200 \mathrm{kc} / \mathrm{s}$, stable à mieux de $10^{-7}$ près), nous permettait de travailler avec des fréquences très stables à $100,50,25,10$ et $5 \mathrm{kc} / \mathrm{s}$. Le champ magnétique était contrôlé par I'intermé-

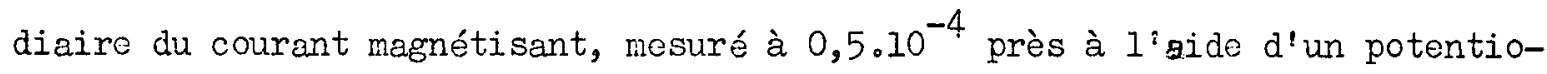
mètre Leeds-Northrup.

La figure 5 montrc un exemple des courbes de résonance ainsi obtenues en portant la quantité $P=\Delta I_{\pi} / I_{\pi_{0}}-\Delta I_{\sigma} / I_{\sigma_{0}}$ en fonction de $H_{0}$ (courant magnétisant); le fréquence était de $10 \mathrm{kc} / \mathrm{s} ; 1$ lilliumination se faisait à l'aide d'une lampe à ${ }^{198} \mathrm{Hg}$. Chaque courbe correspond à une amplitude fixe du champ tournant $\mathrm{H}_{1}$, qui est repérée d'après la tension $\mathrm{V}_{1}$ aux bornes des bobines de radiofréquence; on renarque aux fortes valeurs de $\mathrm{H}_{1}$ un renversement analogue à celui qu'obtenait Brossel dans l'observation de la résonance magnétique du niveau $6^{3} \mathrm{P}_{1}$ du mercure (3).

Nous avons vérifié que les variations $\Delta I_{\pi}$ et $\Delta I_{\sigma}$ ont le bon signe et que leur rapport a bien la valeur théoriquement prévue (cf paragraphe $V$ et tableau II) : 


$$
\begin{aligned}
& \Delta I_{\pi} / I_{\pi_{0}} \simeq-2 \Delta I_{\sigma} / I_{\sigma} \text { dans I'alignenent par }{ }^{204_{\mathrm{Hg}}} \text { (composante 5/2) } \\
& \Delta I_{\pi} / I_{\pi_{0}} \simeq+3 \Delta I_{\sigma} / I_{\sigma_{0}} \text { dans I'alignement par }{ }^{198_{\mathrm{Hg}}} \text { (composante 3/2) }
\end{aligned}
$$

$\Delta I_{\pi}$ est toujours positif puisque les sous--niveaux $\pm 1 / 2$ partiellement vidés par le pornpage se remplissent à résonance $(\Delta \beta>0)$. En utilisant des faisceaux lurnineux très ouverts, on a mesuré à saturation de la résonance sur les maxima de la courbe (ils correspondent à l'égalisation des populations des sous-niveaux $\mathrm{m}_{I}$; $\mathrm{cf}$ troisiène chapitre) des valeurs de $\mathrm{P}$ atteignant 0,10 avec une lampe à ${ }^{198} \mathrm{Hg}$ et 0,18 avec une larape à ${ }^{204} \mathrm{Hg}$. On en déduit que les variations relatives des populations, atteintes par le pompage en aligneinent sur ${ }^{201} \mathrm{Hg}$, dépassent dans les deux cas $16 \%$. (cf paragraphe $\mathrm{V}$ et tableau Ir). On a vérifié que le champ $H_{0}$ auquel se produit la résonance est bien proportionnel à la fréquence du chañ $H_{1}$ à la précision de $1 / 2000$ (Ce contrôle $n^{\prime}$ était pas possible à la précision de $10^{-4}$ de nos mesures de courant magnétisant parce que, en dépit de leur refroidissement par eau, les bobines de Helmholtz produisant $H_{0}$ chauffent et se dilatent légèrement aux fortes intensités de courant). La valeur absolue du chanp était connue avec une précision moindre, d'environ $0,5 \%$, Les résonances étaient observées aux positions prévues $(35,6$ Gauss à $10 \mathrm{kc} / \mathrm{s})$, résultant des mesures antérieures des moments racenétiques nucléaires du mercure (17)(25). Pour obtenir une précision supérieure, il était nécessaire de inesurer directenent le chomp magnétique $H_{0}$ à l'aide d'une résonance de protons.

\section{VIII - Contrôlo du champ directeur H par résonance de protons.}

Pour observer la résonance des protons, nous avons choisi la méthode de Purcell avec le pont en double T utilisé par Soutif (26), tent à cause du faible encombrenent de la tête de mesure, que de la grande souplesse de cette méthode. La tête de mesure était uno simple bobine de fil de Litz de $9 \mathrm{~mm}$ de longueur et de $7 \mathrm{~mm}$ de diariètre, portée per un système 
de 3 crémaillères à angles droits permettant d'explorer le charap magnétique dans un volume assez grand autour du centre des bobines d'Helmholtz. Le générateur était piloté par des quartz aux fréquences de $760.115 \mathrm{c} / \mathrm{s}, 941.994 \mathrm{c} / \mathrm{s}$ et par la suite $800.000 \mathrm{c} / \mathrm{s}$ et $1.000 .000 \mathrm{c} / \mathrm{s}$.

La fréquence de balayage du charnp directeur était choisie de $10 \mathrm{c} / \mathrm{s}$, assez haute pour travailler confortablement, mais assez basse pour pouvoir filtrer les parasites à $50 \mathrm{c} / \mathrm{s}$ et pouvoir ne doper que faiblement l'échantilion d'eau remplissant la tête de mesure (solution de nitrate ferrique à $\mathrm{Fe} / 300$ par litre, dans laquelle le temps de relaxation (27) est de l'ordre de 0,02 seconde). L'amplitude de balayage était de 10 milliGauss, recouvrent juste la largeur à mi-hauteur de le raie de résonance $(\sim 40 \mathrm{c} / \mathrm{s}$ ). On obtenait ainsi aux points où l'homogénéité du champ était assez bonne, un rapport signal/bruit supérieur à 20, permettant le contrôle de la constante du champ à $10^{-6}$ près.

Nous avons pu vérifier à cette précision la stabilité à court terme de l'alimentation haute tension Varian, qui présente par ailleurs une dérive lente peu gênante.

Pour mesurer l'homogénéité du chanp $\mathrm{H}_{0}$ dans l'espace, nous avions étalonné le dispositif potentiométrique permettant d'inposer à l'alimentation Varian do faibles écarts de courant autour d'une valeur moyenne, et nous lisions ainsi directement l'écart nécessaire pour recentrer le signal de résonance des protons sur l'écran de l'oscillographe après un déplacement de la tête de mesure. En supposant les bobines de chanp parfaitement circulaires et indéformables, la carte du champ ainsi tracée dépend théoriquenent

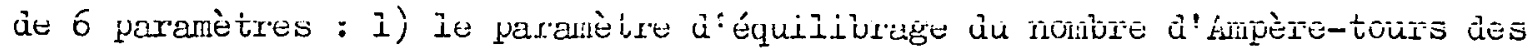
deux bobines (addition d'une forte résistance en parallèle avec l'une d'elles) 2) les 3 paramètres de distance et de parallélisme des plans moyens de chaque bobine - 3) les 2 paranètres de translation des bobines l'une par rapport à l'autre parallèlement à leur plan moyen, permettant d'assurer la coincidence de leurs axes. En procédant par approximations successives, nous sommes 
arrivés à obtenir une carte de chenp très semblable à la meilleure carte de champ théorique (annulant les temes du second ordre; cf annexe II); c'està-dire que nous mesurions des écarts relatifs du champ $H_{0}$ atteignant au maximurn $\pm 3 \cdot 10^{-6}$ ou $\pm 4 \cdot 10^{-5}$ respectivenent à l'intérieur des cubes de $2 \mathrm{~cm}$ d'arête ou $4 \mathrm{~cm}$ d'arête, ayant même centre que les bobines de charap, et correspondant aux volumes des cellules à vapeur de nercure utilisćes.

\section{IX - Les bobines et le champ $\mathrm{H}_{1}$ de radiof réquence.}

Les fréquences employées pour observer les résonances nucléaires du mercure $n^{\prime}$ ont jamais atteint $200 \mathrm{kc} / \mathrm{s}$, et nous $n^{i}$ avons jamais eu besoin de puissances importantes. Ceci $n$ 'inposait aucune exigence particulière au circuit de radiofréquence constitué de deux bobines minces d'axe vertical, en position d'Helmholtz. Nous nous sormes intéressés cependant à l'homogénéité du champ $H_{I}$ dans l'espace. C orme nous opérions à basse fréquence, nous avons pu tester expérimentalement cette homogénéité en nesurant la tension induite aux bornes d'une petite bobine (diamètre extérieur $1 \mathrm{~cm}$, longueur $1 \mathrm{~cm}$, 800 tours de fil de 10/100 min) d'axe vertical également, et portée par le dispositif de 3 crénaillères à angles droits.

La tension induite dans cette sonde se montait à quelques millièmes seulement de la tension appliquée aux bornes du circuit de radiofréquence; et cependant la tension parasite induite par capacité était réduite à moins de $1 \%$ de la tension utile induite par le champ nagnétique $\mathrm{H}_{1}$ grâce aux trois précautions suivantes : a) connexions extrêmernent courtes dans la tête de nesure entre la bobine et le câble coaxial la reliant au voltmètre électronique - b) connexion de l'armature externe du coaxial avec le fil venant de la couche externe de la bobine - c) blindage de la bobine par un petit cylindre de laiton, relié à l'arnature externe du coaxial, et fendu le long d'une génératrice pour empêcher la fermeture des courants de Foucault. Nous avons également construit une seconde sonde identiquo à la première, et 
observé que son approche au voisinage immédiat de la première sonde perturbait d'environ $1 \%$ seulement la tension induite. Conme nous ne nous intéressions qu'aux variations du champ $\mathrm{H}_{1}$, nous avions ainsi la certitude de les mesurer effectivement à $0,5 \%$ de $l_{a}$ valeur de $H_{1}$, limite imposée par la lecture du millivol tmètre électronique.

Ce dispositif nous a perrais de reconnaître d'abord que les tôles métalliques placées au voisinage des bobines de radiofréquence n'ont qu'un effet négligeable sur le champ $H_{1}$, dès qu'elles ne sont pas perpendiculaires ¿̀ I'exe du circuit. Par contre, l'homogénéité de $\mathrm{H}_{1}$ est très mauvaise si les deux bobines du circuit de radiofréquence ne sont pas enroulées avec une parfaite régularité, assurent leur totale symétrie. Un prenier système de bobines de faible dianètre (60 tours de $11 \mathrm{~cm}$ de diamètre moyen) était à cet égard déplorable. Nous l'avons remplacé par deux nouvclles bobines soigneusenent enroulées comprenant chacune 54 tours de $24 \mathrm{~cm}$ de dianètre moyen. L'inhomogénéité résiduelle au centre de ce nouveau circuit, dans le volurne des cellules à vapeur de mercure utilisées, est alors inappréciable avec notre dispositif de nesure. Mais nous pouvions mesurer les variations de $\mathrm{H}_{1}$ à des distances plus grandes; elles sont sensiblement égales aux variations théoriques; et nous pouvons raisonnablenent penser que les variations de $\mathrm{H}_{1}$ au voisinage du centre sont égalenent du mêne ordre de grandeur que les variations théoriques ( $\mathrm{ff}$ annexe II). Cela correspond à une inhonogénéité d'environ $10^{-4}$ dans un cube de $2 \mathrm{cnl}$ d'arête et $10^{-3}$ dans un cube de $4 \mathrm{~cm}$ d'arête.

La réalisation de cette homogénéité dans l'espace s'est révélée inutile en fin de compte, conme nous le verrons par la suite (quatrième chapitre). Il est important par contre de contrôler la stabilité dans le temps du champ $\mathrm{H}_{1}$, pendant le tracé de chaque courbe de résonance, et de mesurer son amplitude on valeur relative. Un simple millivoltnètre électronique est utilisé à cet usage; il est branché en permanence à l'entrée du câble coaxial alimentant le circuit de radiofréquence, et mesure à environ $1 \%$ près la tension $\mathrm{V}_{1}$, proportionnelle à $\mathrm{H}_{1}$, tant qu'on reste à fréquence 
fixe. Lorsque nous tracerons des courbes à fréquence variable (cf paragraphe suivant), l'excursion de fréquence $A \nu$ sera toujours suffisament faible $\left(\Delta y / \gamma<10^{-3}\right)$ pour que cela reste vrai.

\section{$X$ - Mesures en chanp fixe.}

Pour augmenter la précision des déterminations des fréquences de résonance nucléaire du nercure, il était nécessaire de nesurer en perma." nence le chanp magnétique $H_{0}$ à I'aide du signal de rósonance des protons. La détection radioélectrique utilisée pour celle-ci ne permettant pas de changer facilement sa fréquence, nous étions conduits à travailler à champ fixe et à balayer en fréquence les résonances du mercure.

Ce balayage de fréquence exige qu'on procède à des mesures précises de la fréquence variable. Nous utilisons un fréquencenètre à décades, qui compte le nombre de périodes pendant 10 secondes et peut donc fournir la précision absolue de $0,1 \mathrm{c} / \mathrm{s}$, soit une précision relative de $10^{-6} \mathrm{si} \mathrm{la}$

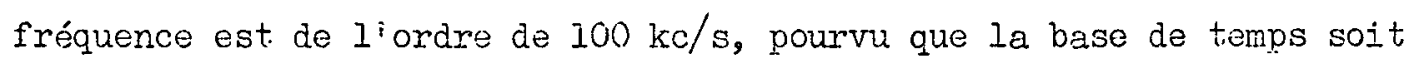
définie avec une précision suffisante. Celle-ci est déínie à partir d'un quartz thermostaté à $100 \mathrm{kc} / \mathrm{s}$, dont on peut contrôler et corriger la fréquence en permanence par comparaison aver la fréquence étajon de $l^{i}$ émetteur de Droitwich. On mesure et on naintient constant le chanp $\mathrm{H}_{0}$ à $10^{-5}$ près grâce à la résonance de protons, dont la tête de raesure est placée en un point fixe voisin de la cellule à vapeur de mercure. On a vérifié que le faible champ de balayage à $10 \mathrm{c} / \mathrm{s}$ n'apporte aucune perturbation aux signaux

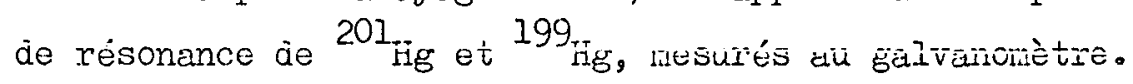

Pour alimenter les bobines de radiofréquence, nous disposions d'un classique générateur à self et capacité variable, nais de très bonne qualité, et dont la stabilité en fréquence sur de courts intervalles de temps dépassait largement $10^{-5}$, atteignant presque $10^{-6}$. C:est avec ce générateur que nous avons tracé toutes les courbes de résonance de ${ }^{201} \mathrm{Hg}$ 
tant en alignement (courbes analogues à celle de la figure 5) qu'en orientation; les largeurs de raie sont telles que la stabilité de ce générateur est suffisante.

Dans le cas de la vapeur de ${ }^{201}$ Hg orientée, les résonances ne présentent pas le renversement central aux fortes valeurs de $\mathrm{H}_{1}$; elles sont en outre plus étroites. La fígure 6 montre quelques courbes obtenues par illumination en lumière circulaîre avec une lampe à ${ }^{204} \mathrm{Hg}$ (composante 201-5/2). Bien que le processus d'orientation soit plus efficace que celui d'alignement, la réduction de l'intensité lumineuse, résultant de l'interposition du prisme de Nicol et de la lame quart d'onde, conduit à des effets beaucoup moins inportants qu'en alignement; on mesure à saturation $P=0,03$ ce qui correspond à un enrichissement d'environ $3 \%$ du niveau $3 / 2$ (cf paragraphe $V$ et tableau III). Le rapport signal/bruit est mauvais, ce qui explique la dispersion des points expérimentaux.

On a observé avec la même lampe à ${ }^{204} \mathrm{Hg}$ l'orientation de ${ }^{199} \mathrm{Hg}$. Comme nous I'avons déjà signalé (paragraphes IV et V, tableau I), lo taux de polarisation de la lunière de résonance optique est constant dens ce cas; et la détection se fait en mesurant son intensité, qui est proportionnclle à la population $\beta$ du niveau $m_{T}=-1 / 2$ (qui est vidé par le pompage en $\sigma^{+}$). A la différence du cas de ${ }^{201} \mathrm{Hg}$, les courbes de résonance (figure 7) sont beaucoup plus fines et les taux d'orientation bcaucoup plus importants : les variations relatives des populations sont de $34 \%$ dans le cas de la figure 7; et on a observé plus de $50 \%$. Ces deux effets sinultanés montrent que le temps de relaxation de ${ }^{199} \mathrm{Hg}$ est beaucoup plus long que celui de $20 \mathrm{I}_{\mathrm{Hg}}$; nous reviendrons sur ce point au quatriène chapitre.

Les courbes de résonance de ${ }^{199} \mathrm{Hg}$ sont nêne tellement fines qu'il n'est plus possible do les tracer correctement avec le générateur à self et capacité décrit plus haut. Ayant seulenent repéré leur position, nous avons fait tailler des quartz aux fréquences nécessaires de $142,62 \mathrm{kc} / \mathrm{s}$ 
et $178,27 \mathrm{kc} / \mathrm{s}$ (correspondant à la résonance de protons à $800 \mathrm{kc} / \mathrm{s}$ et $I \mathrm{Mc} / \mathrm{s})$; ils nous ont servi à piloter un générateur dont la stabilité dépassait ainsi largement $10^{-6}$. Les résonances sont alors décrites par ajustement de la fréquence du générateur à l'aide d'un condensateur variable inséré dans le circuit du quartz. C'est dans ces conditions qu'ont été tracées les courbes de la figure 7; les écarts des points expérimentaux mesurent alors la limite de stabilité et de contrôle du champ directeur $\mathrm{H}_{0}$. Notons que ce sont des courbes de Lorentz même aux fortes amplitudes du champ de radiofréquence $\mathrm{H}_{1}$, puisqu'il n'y a que deux sous-niveaux mis en jeu dans la résonance magnétique; nous le vérifions expérimentalement.

\section{XI - Les moments magnétiques nucléaires.}

La stabilité et la finesse des signaux obtenus nous a permis une mesure précise des moments magnétiques nucléaires des isotopes impairs du mercure. Cette mesure était intéressante, car la plupart des valeurs adnises jusqu'ici n'étaient calculées qu'à partir de mesures optiques de structures hyperfines (17). Seuls Proctor et $Y u$ (25) avaient fait une mesure directe par résonance magnétique sur une solution de nitrate mercureux; mais i.ls n'avaient pu obscrver que la résonance de ${ }^{199} \mathrm{Hg}$.

Pour cette mesure, il ne suffit plus de contrôler la constance du champ $\mathrm{H}_{\mathrm{o}}$, mais il faut comparer les fréquences de résonance du mercure et du proton dans le même champ. Le plus simple est alors de placer la tête de mesure de résonance de protons en un point voisin de la cellule à vapeur de mcroure, où le chemp magnéticnue retrnuve exactement la même valeur qu'au centre de la cellule (qui coincide avec le centre géométrique du champ); mais en ces points, I'homogénéité de champ n'est pas toujours bonne, et la résonance des protons peut être élargie, ce qui diminue la précision. Il y a donc souvent avantage à placer la tête de mesure en un point différent, et à mesurer avec soin $l^{2}$ écart de champ correspondant par rapport au champ au centre. Nous avons utilisé l'une et l'autre technique. Nous avons fait les 
mesures un grand nombre de fois en utilisant diverses cellules, des intensités lumineuses d'irradiation très différentes, et, pour ${ }^{201} \mathrm{Hg}$, dans des cas d'orientation ou d'alignement.

Nous avons aussi utilisé une cellule remplie d'un mélange à parts égales de ${ }^{201} \mathrm{Hg}$ et ${ }^{199} \mathrm{Hg}$, et sur laquelle nous pouvions observer les deux résonances pour déterminer directement le rapport de leurs fréquences dans des conditions strictement identiques.

Nous avons également fait les mesures dans quatre champs magnétiques différents. Nous avons utilisé en particulier pour piloter la résonance de protons des quartz taillés aux fréquences de $1 \mathrm{Mc} / \mathrm{s}$ et $800 \mathrm{kc} / \mathrm{s}$; nous remplaçons alors le générateur à quartz du fréquencemètre par un multivibrateur à $100 \mathrm{kc} / \mathrm{s}$ synchronisé par le générateur de résonance de protons. Ainsi le fréquencemètre mesure directement le rapport des fréquences de résonance du mercure et du proton, sans être affecté par de possibles fluctuations du quartz,

En définitive, la précision de nos mesures est limitée dans le cas de ${ }^{201} \mathrm{Hg}$ par la largeur des résonances et le bruit du signal optique observé; dans le cas de ${ }^{199} \mathrm{Hg}$ elle n'est limitée que par nos techniques de mesure et la stabilité du champ $H_{0}$. Dans les deux cass, la précision doit pouvoir être améliorée; les résultats actuels sont les suivants :

a) Le rapport des moments nucléaires ne dépend pas de la correction diamagnétique qui est la même pour les deux isotopes :

$$
\frac{\gamma_{201}}{\gamma_{199}}=-0,3691385( \pm 15) \text { ou } \frac{\mu_{201}}{\mu_{199}}=-1,107416( \pm 5)
$$

ce rapport diffère sensiblenent des valeurs mesurécs spectroscopiquement à partir des écarts hyperfins (17). Des mesures précises des écarts hyperfins, récemment obtenues, mettent en évidence, par comparaison avec notre mesure, l'existence d'anomalies de structure hyperfine importantes dépassant 1/1000 : 
la méthode des jets atomiques appliquée à. 1 'état métastable $6^{3} \mathrm{P}_{2}$ (28) conduit à la valeur $3 \mathrm{~A}_{201} / \mathrm{A}_{199}=-1,109124$; et la méthode de double résonance conduit pour l'état $63 \mathrm{P}_{1}(29)$ à la valeur $3 \mathrm{~A}_{201} / \mathrm{A}_{199}=-1,109040$.

b) Nous donnons les autres valeurs non compte-tenu de la correction diamagnétique, qui d'après le calcul de Lamb (30) atteindrait $0,96 \%$ :

$$
\frac{\gamma_{201}}{\gamma_{\mathrm{H}}}=-0,0658066( \pm 3) ; \quad \frac{\gamma_{199}}{\gamma_{\mathrm{H}}}=0,1782706( \pm 3)
$$

en prenant $\gamma_{\mathrm{H}}=26751,3( \pm 0,2)(31)$, on obtient :

$$
\gamma_{201}=-1760,41( \pm 2) ; \quad \gamma_{199}=4768,97( \pm 4)
$$

en prenant $\mu_{H}=2,79275( \pm 3) \mu_{n}$ (32)(33), on obtient :

$$
\mu_{201}=-0,551344( \pm 9) \mu_{n} ; \mu_{199}=0,497865( \pm 6) \mu_{n}
$$

Cette valeur de $\mu_{199}$ est inférieure de $0,3 \%$ à celle mesurés por Proctor et $\mathrm{V}_{u}$ (25) dans une solutior de nitrate mercureux. 


\section{TROISIEME CHAPITRE}

\section{LES METHODES D'ÉTUDE DE LA RELAXATION}

\section{XII - Les temps de relaxation et la méthode statique.}

En dehors de la détermination des fréquences de résonance, les courbes expérimentales, que nous avons montrées au chapitre précédent, permettent l'étude des phénomènes de relaxation à partir des mesures de largeurs de raie. C'est ce que nous appelons la méthode statique d'étude de la relaxation, par opposition à la méthode dynamique qui utilise l'observation des phénomènes transitoires (cf paragraphe suivant). L'interprétation quantitative de ces deux types de phénomènes suppose une théorie permettant de les calculer. Les calculs correspondants, utilisant le formalisme de la matrice densité, seront développés en détail à la fin de ce chapitre, et justifieront les résultats dont nous faisons état dans ces deux premiers paragraphes pour exposer les méthodes d'étude expérimentales.

Nous réservons pour le quatrième chapitre l'étude des origines de la relaxation; et dans le présent chapitre, nous décrivons la relaxation de façon purement phénoménologique, à la manière de Bloch (34), à l'aide des deux temps de relaxation longitudinale $\mathrm{T}_{1}$, et de relaxation transversale $\mathrm{T}_{2}$. C'est-à-dire que nous supposons l'existence d'une probabilité $1 / T_{1}$ par unité de temps pour que l'état d'orientation des atomes soit modifié par la relaxation, et d'une probabilité $1 / T_{2}$ pour qu'ils soient arrachés par la relaxaiiư à là süperposition cohćrente d'états dans laquelle les fait passer l'interaction avec le champ de radiofréquence. Cette hypothèse conduit dans le cas de ${ }^{199} \mathrm{Hg}$, où il n'y a que 2 sous-niveaux Zeeman, aux équations de relaxation les plus générales possibles; les conclusions que nous en tirons sont valables en tout état de cause, et se trouvent bien vérifiées par l'expérience. Dans le cas de ${ }^{201} \mathrm{Hg}$ par contre, il y a 4 sous-niveaux Zeeman, et la descrip- 
tion de la relaxation à I'aide de 2 constantes $\mathrm{T}_{1}$ et $\mathrm{T}_{2}$ seulement ne peut être considérée que comme un modèle approché; on ne s'étonnera pas que certaines de ses propriétés ne soient pas vérifiées par l'expérience. Mais ce modèle simplifié nous sera un guide utile pour étudier les origines de la relaxation (quatrième chapitre), et déterminer ainsi à partir de quelles hypothèses peut être bâti un modèle plus exact.

Outre l'action du champ toumant $\mathrm{H}_{1}$ et do la relaxation, il nous faut également tenir compte de l'action de la lumière de pompage optique. C'est comme la relaxation un phénomène aléatoire; mais nous avons vu (paragraphe VI) que, si l'excitation lumineuse n'est pas du type ''broad line'", la probabilité par unité de temps pour qu'un atome absorbe un photon dépend du sous-niveau $m_{I}$ où il se trouve placé; et nous ne savons plus ce que vaut cette probabilité d'excitation lorsque, sous l'action du champ $\mathrm{H}_{1}$, l'atome est passé dans une superposition cohérente d'états. Nous verrons à la fin de ce chapitre que les calculs de moyenne sur tous les atomes, effectués à 1 'aide de la matrice densité, sont équivalents, dans les cas d'orientation du ${ }^{199} \mathrm{Hg}$ et d'alignement du ${ }^{201} \mathrm{Hg}$, à l'existence d'une probabilité d'excitation définie valant $1 / T_{p}$ (cf équations (VI-4) et (VI-5)). Les atomes peuvent être arrachés à la superposition cohérente d'états au niveau fondamental par deux processus aléatoires indépendants : la relaxation de probabilité $1 / T_{2}$, et l'oxcitation au niveau d'énergie supérieur

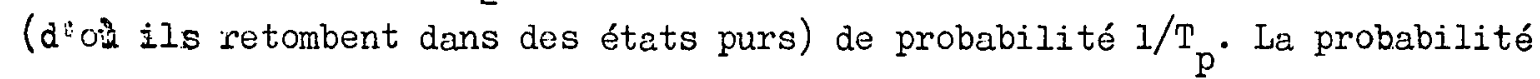
totale d'arrachement à l'état cohérent est la somme de ces deux probabilités :

$(X I I-10)$

$$
\frac{1}{\tau_{2}}=\frac{1}{T_{2}}+\frac{I}{T_{p}}
$$

C'est ce "temps de cohérence" $\tau_{2}$ qui joue dans nos expériences lo rôle de temps de relaxation transversale apparent. 
La lumière a également pour effet de modifier l'état d'orientation des atomes par le jeu des probabilités de retombée de l'état excité; c'est le processus même du pompage optique. On conçoit donc que les phénomènes étudiés soient décrits également avec un temps de relaxation longitudinale apparent $\tau_{1}$, dépendant de l'intensité-lumineuse, qui est défini par :

$$
\frac{1}{\tau_{1}}=\frac{1}{T_{1}}+\frac{k}{T_{p}}
$$

Le coefficient $k$ dépend des probabilités de transition optique : $k=2 / 3$ dans le cas d'orientation de ${ }^{199} \mathrm{Hg}$ avec une lampe à ${ }^{204} \mathrm{Hg}$ (cf formules $(\mathrm{XV}-22)) ; \mathrm{k}=0,36$ ou 1,04 dans le cas d'alignement de ${ }^{201} \mathrm{Hg}$ avec une lampe à ${ }^{204} \mathrm{Hg}$ ou à ${ }^{198} \mathrm{Hg}$ (cf formules (XVI-33)). Ce sont ces temps apparents $\tau_{1}$ et $\tau_{2}$ qui sont accessibles directement à nos mesures; nous allons le voir d'abord dans le cas de la méthode statique.

Lorsqu'on trace un réseau de courbes de résonance (cf figures 5,6 et 7 ), on trouve que la largeur à mi-hauteur I (nous la supposerons toujours exprimée en fréquence) croît avec l'intensité du champ tournant $H_{1}$. L'interprétation de cet élargissement est simple : il est dû au raccourcissement de la durée de vie des sous-niveaux Zeeman par absorption et émission induite à la fréquence $\omega / 2 \pi$ du champ $\mathrm{H}_{1}$. Si l'on extrapole cette largeur à $\mathrm{H}_{1}$ nul, la largeur limite obtenue ne doit plus dépendre que des autres causes qui limitent cette durée de vie (relaxation et absorption de photons), c'est-à-dire du "temps de cohérence!" $\tau_{2}$ défini plus haut. La théorie facilite l'extrapolation en indiquant que ie carré de ia iargeuri est une fonction linéaire du carré de l'amplitude du champ tournant :

$$
(\mathrm{XII}-12) \quad L^{2}=\left(\frac{I}{\pi \tau_{2}}\right)^{2}+\mathrm{K} \cdot \mathrm{H}_{1}^{2}
$$

(cf formules $(\mathrm{XV}-24)$ et $(\mathrm{XVI}-38)$ ) 
Expérimentalement, on mesure la tension $V_{I}$ aux bornes du circuit de radiofréquence, proportionnelle a $H_{1}$; et on vérifie que $L^{2}$ est bien représenté par une droite en fonction de $V_{1}^{2}$. L'ordonnée à l'origine de cette "droite des largeurs" vaut $\left(1 / \pi \tau_{2}\right)^{2}$; on en déduit $\tau_{2}$. Les figures 8 et 9 nous montrent des exemples de ces"droites des largeurs" (droites $n^{0} 1$ ). La figure 8 correspond au réseau tracé sur la figure 7 dans le cas de ${ }^{199}{ }_{\mathrm{Hg}}$ orienté avec une lampe à ${ }^{204} \mathrm{Hg}$; on mesure $1 / \pi \tau_{2}=0,90 \mathrm{c} / \mathrm{s}$ et $\tau_{2}=0,35$ seconde. La figure 9 correspond à un réseau analogue à celui de la figure 5 dans le cas d'alignement de ${ }^{201} \mathrm{Hg}$ avec une lampe à ${ }^{198} \mathrm{Hg}$, mais avec une intensité lumineuse plus forte; on mesure $1 / \pi \tau_{2}=22 \mathrm{c} / \mathrm{s}$ et $\tau_{2}=0,014$ seconde.

D'après la formule (XII-10), cette largeur limite $1 / \pi \tau_{2}$ doit encore dépendre de l'intensité lumineuse $\underset{\mathcal{P}}{ }$, à laquelle $1 / \mathrm{T}_{\mathrm{p}}$ est proportionnel. Pour le vérifier, on réalise diverses valeurs de $\bar{F}$ variant dans des rapports connus, en interposant sur le faisceau d'irradiation des grilles à mailles serrées qui réduisent son intensité sans modifier sa configuration géométrique (les rayons plus ou moins inclinés sur l'axe, ct qui n'ont peut-être pas la même efficacité, sont ainsi affaiblis d'égale manière). Pour chaque valeur de $\Phi$ on trace un réseau de courbes de résonance et une droite des largeurs permettant d'extrapoler une largeur limite $1 / \pi \tau_{2}$. On vérifie que ces largeurs $1 / \pi \tau_{2}$ sont bien représentées par une droite en fonction de $\Phi$; et la nouvclle largeur limite, extrapolée à intensité lumincuse $\oint_{1}$ nulle, mesure $1 / \pi T_{2}$. La figure 10 nous montre un exemple de cette vérification et de cette seconde extrapolation dans un cas d'alignement de ${ }^{201} \mathrm{Hg}$; on obtient $1 / \pi \mathrm{T}_{2}=8,0 \mathrm{c} / \mathrm{s}$, ou $\mathrm{T}_{2}=0,04$ seconde.

Ayant ainsi déterminé $T_{2}$, nous pouvons à l'aide de la formule (XII-10) déterminer dans chaque cas particulier la part de la largeur due aux photons $1 / \pi \mathrm{T}_{\mathrm{p}}$. Cette largeur a atteint en alignement une valeur maximum de l'ordre de $30 \mathrm{c} / \mathrm{s}$, ce qui correspond pour $T_{p}$ à un minimum de l'ordre de 0,01 seconde. On en déduit une mesure indirecte du temps de relaxation 
longitudinale $\mathrm{T}_{1}$ : les résultats des paragraphes $\mathrm{V}$ et VI (tableaux $\mathrm{I}$, II et III) permettent de calculer le rapport $T_{1} / T_{p}$ à partir du signal maximum observé à saturation de la résonance; connaissant maintenant $\mathrm{T}_{\mathrm{p}}$, nous pouvons en déduire $\mathrm{T}_{1}$. Il est encore possible de déterniner $\mathrm{T}_{1}$ d'une autre manière, si l'on connait la valeur absolue de l'amplitude $\mathrm{H}_{1}$ (et nous verrons que l'observation des transitoires de ${ }^{199} \mathrm{Hg}$ nous donne une mesure précise du rapport $\left.\mathrm{V}_{1} / \mathrm{H}_{1}\right)$ : on peut alors mesurer la pente de la droite des largeurs en fonction de $\mathrm{H}_{l}^{2}$; cette pente est connue theoriquement en fonction du rapport $\tau_{1} / \tau_{2}$ (cf formules $(X V-24)$ et $(X V I-38)$ ) qu'elle permet ainsi de mesurer; on en déduit $\tau_{1}$, puis $T_{1}$ à l'aide de la formule (XII-ll).

Cette méthode statique de mesure des temps de relaxation présente deux inconvénients : a) par sa longueur : pour pouvoir procéder à la double extrapolation décrite ci-dessus, il faut tracer un très grand nombre de courbes, ce qui exige un temps considérable; plusieurs journées de nesure sont nomalement nécessaires pour déterminer une valeur de $\mathrm{T}_{2}-\mathrm{b}$ ) par sa faible précision : il est en effet difficile de garantir une parfaite stabilité de la source lumineuse pendant des temps aussi longs, et cela ne fait qu'augmenter les erreurs propres à toute méthode d'extrapolation; au total la précision de mesure des temps $\mathrm{T}_{2}$ atteint à peine $10 \%$. Les erreurs $\mathrm{s}^{\prime}$ accumulent bien davantage encore dans la suite d'opérations nécessaires à la détermination indirecte de $T_{1}$, d'où l'on ne peut tirer que des valeurs très approximatives.

XIII - La mélhüúe ḋy̆amiquic - Obscrvation des transitnires de ${ }^{199} \mathrm{Hg}$.

La méthode dynamique, au contraire de la méthode précédente, permet des mesures rapides et directes de $\mathrm{T}_{2}$ et de $\mathrm{T}_{1}$, par observation des phénomènes transitoires qui se produisent lorsqu'on impose de brusques modifications à l'intensité Iumincuse orientatrice $\Phi$, ou au chemp tournant $H_{1}$. 
Cette méthode est donc bien préférable à la précédente. Mais, pour les raisons que nous verrons, nous avons pu jusqu'ici l'appliquer seulement au cas de ${ }^{199} \mathrm{Hg}$; nous l'exposons done uniquement dans ce cas-là. Pour faciliter l'exposé, nous supposons toujours qu'il s'agit d'une irradiation en polarisation $\sigma^{+}$, qui tend à diminuer la population $\beta$ du sous-niveau $m_{I}=-1 / 2$.

Nous avons utilisé cette méthode uniquement dans lo cas où ${ }^{199} \mathrm{Hg}$ est excité par la composante $1 / 2$ avec une lampe à ${ }^{20}{ }^{H} \mathrm{Hg}$, en mesurant I'intensité de la lumière totale de fluorescence, constamment dépolarisće, qui est proportionnelle à la population $\beta$ du sous-niveau - $1 / 2$. Mais les mêmes phénomènes seraient observables sans grand changement en irradiation simultanée par les deux composantes $1 / 2$ et $3 / 2$, à condition de ne détecter que la lumière de fluorescence de polarisation $\pi$, dont l'intensité est dans tous les cas proportionnelle à la seule population $\beta$. La méthode comprend en réalité plusieurs variantes qui se complètent et que nous étudions successivement. Dans tous les cas, les observations sont faites en envoyant lo courant d'un seul photomultiplicateur à travers la résistance d'entrée d'un oscillographe à entrée continue : le signal observé sur l'écran en fonction du temps est, à intensité constante de la lampe, proportionnel à la population $\beta$ du niveau - 1/2. L'échelle de temps est indiquée sur les oscillogrammes que nous présentons par des signaux triangulaires à basse fréquence.

A - Transitoires du pompage optique en I'absence de radiof réquence. Les deux premières parties de la méthode sont tout à fait analogues aux expériences réalisées par Franzen sur le rubidium (35). On étudie d'abord la dynamique du pompage optique, c'est-à-dire le temps nécessaire pour atteiñủie l'orientation stationnaire qui résulte de la compétition entre les photons orientateurs et la relaxation. Le faisceau d'irradiation $\sigma^{+}$garde une intensité constante; mais il est initialement nasqué par un obturateur : le signal observé est nul, et la vapeur non irradiée n'est pas orientée. Au temps $t_{1}$, on ouvre brusquement l'obturateur (cf figure 1la); l'intensité de lumière réémise prend 
la valeur $I_{1}$ due à des atomes non orientés $(\beta=I)$; puis elle diminue jusqu'à la valeur limite stationnaire $I_{0}$ en suivant une loi exponentielle dont on démontre facilement (cf paragraphe XV-B) que la constante de temps est le temps $\tau_{I}$ défini plus haut. (Sur la figure 11 a, on mesure $\tau_{1}=1,2 \mathrm{~s}$ ). L'état limite est attuint sous l'action de deux processus aléatoires indépendants dont les probabilités respectives par unité de temps sont $1 / T_{1}$ (désorientation) et $2 / 3 T_{p}$ (passage du niveau $-1 / 2$ au niveau $+1 / 2$ par absorption d'un photon); on s'en approche avec une probabilité qui est la somme de ces deux probabilités $1 / \tau_{1}=1 / T_{1}+2 / 3 T_{p}$; on retrouve bien la formule (XII-II). On vérifie expérimentalement que $1 / \tau_{1}$ est une fonction linéaire de l'intensité lumineuse $\Phi$ (cf figure 14). On pourrait ainsi mesurer $T_{I}$ par extrapolation à intensité Iumineuse nulle; mais il existe un procédé plus direct.

\section{B - Relaxation dans le noiv.}

On peut étudier directement l'action de la relaxation seule, dans le noir : l'intensité $I_{0}$ étant atteinte, on interrompt alors au temps $t I^{\prime} i r-$ radiation pour une duréo courte $\Delta t$ (figure llb). L'intensité réémise tombe à zéro; mais sous l'effet de la relaxation la population $\beta$ tend vers la valeur 1, d'équilibre thermique, suivant une loi exponentielle de constante de temps $T_{I}$ (courbe ponctuée superposée à l'oscillogramme). Au temps $t+\Delta t$, l'intensité lumineuse part donc d'une valeur intermédiaire entre $I_{1}$ et $I_{0}$ (point A) pour décrire à nouveau l'exponentielle de pompage. Si on fait varier $\Delta t$, le point initial A décrit la courbe exponentielle de relaxation dans le noir, qui se trouve matérialisée par la superposition photographique de la figure llc ( l'échelle des ordonnées est multipliée par 2 environ, pour ne représenter que la partie utile entre $I_{0}$ et $\left.I_{1}\right)$; on mesure ainsi directement sur 1'oscillogramme $\mathrm{T}_{1}=2$ secondes. La figure $11 \mathrm{~d}$ représente l'application de la méthode avec une autre cellule où $T_{1}=13$ secondes ( 1 a courbe isolée est obtenue après un temps très long dans le noir; elle permet de tracer l'asymptote horizontale de l'exponentielle). 


\section{C - Transitoires de la résonance magnétique;}

Cette troisième partie de la méthode est semblable aux expériences réalisées par Torrey (36) avec la résonance des protons dans l'eau. Lorsque les spins nucléaires sont soumis brusquenent au champ tournant $\mathrm{H}_{1}$, leurs mouvements de "nutation" s'effectuent tous en phase, et ils sont alors observables macroscopiquement, tant que la relaxation transversale ne les a pas totalement déphasés. En d'autres termes, l'interaction avec $H_{l}$ fait passer les atomes d'un état pur à une superposition cohórente d'états $\psi(t)=\Lambda(t) \cdot \Psi_{+1 / 2}+B(t) \cdot \psi_{-1 / 2}$; les interactions entre atomes étant faibles, l'amplitude $B(t)$ varie en phase pour tous les atomes, et d!après le calcul de Rabi (37), elle suit la loi en $\sin (1 / 2) \sqrt{\omega_{1}^{2}+\Delta \omega^{2}} \cdot t\left(\omega_{1}=\gamma H_{1} ; \Delta \omega=\omega-\gamma H_{0}\right)$, tant que la relaxation $n$ a pas encore arraché les atomes à cette superposition cohérente. La population $\beta=B(t)^{2}$ a donc une variation sinusoidale de pulsation double $\sqrt{\omega_{1}^{2}+\Delta \omega^{2}}$, amortie par la relaxation. C'est ce que montre exactement le calcul du paragraphe XV; et c'est ce que nous observons sur les figures 12 en enregistrant les variations de I'intensité lumineuse lorsque, ayant atteint I'orientation limite $I_{0}$, on sounet bravquareat lea stosies à lootion de $H_{1}$ : l'intensité lumineuse réémise rejoint alors sa nouvelle valeur d'équilibre par l'intermédiaire d'une variation sinusoidale transitoire.

-a) La nouvelle valeur d'éguilibre correspond à une dósorientation partielle ou totale des atomes. Si à l'équilibre la résonance est saturće $\left(\Delta \omega=0, H_{1}\right.$ très grand; figures $12 b$ et $\left.12 c\right)$, la désorientation est totale et l'intensité lumineuse retrouve la valeur $I_{1}$ due à des atomes désorientés. La désorientation n'est que partielle au contraire si l'on est écarté de la résonance ( $\Delta \omega \neq 0$; figure $12 d$ ), ou si, à résonence, I'amplitude du champ $H_{I}$ est trop faible (figure 12a; la seconde partie de cette figure est obtenue par suppression du champ $\mathrm{H}_{1}$ : on observe à nouveau une exponentielle de pompage optique de constente de temps $\tau_{l}$, analogue à celles des figures lla et llb). 
-b) Pour l'interprétation des transitoires, on notera que les figures $12 \mathrm{a}, 12 \mathrm{~b}$ et $12 \mathrm{c}$ ont été obtenues à résonance, avec des amplitudes croissantes du champ tournant $H_{1}$ (repérées d'après l'amplitude de la tension $\mathrm{V}_{1}$ aux bornes $\mathrm{du}$ circuit de radiofréquence, proportionnelle à $\left.\mathrm{H}_{1}\right)$. On vérifie à résononce $(\Delta \omega=0)$ que, conformément au calcul, l'enveloppe exponentielle de la sinusoide est indépendante de $V_{1}$, et que la fréquence de la sinusoide est bien proportionnelle à $V_{1}$ (Le rapport $V_{1} / H_{1}$ ainsi mesuré est en bon accord avec l'ordre de grandeur calculé d'après le coefficient de self $\mathcal{L}$ du circuit, le diamètre $\mathrm{D}$ et le nombre de tours $\mathrm{n}$ de chaque bobine $\left.: \mathrm{V}_{1} / \mathrm{H}_{1}=\mathrm{D} \cdot \mathscr{L} \cdot \omega / 0,9 n\right)$. On vérifie également lorsqui on s'écarte de la résonance que la sinusoide amortie a sa fréquence augmentée, conformément à la formule $\sqrt{\omega_{1}^{2}+\Delta \omega^{2}}$ (figure 12d, obtenue avec la même amplitude $H_{1}$ que la figure $12 \mathrm{c}$; cf vérification détaillée au paragraphe XV-D). Les mesures se font au centre de la résonance : la fréquence de la sinusoide $(\gamma / 2 \pi) \mathrm{H}_{l}$ nous permet alors une mesure précise de $\mathrm{H}_{1}$ en valeur absolue; et la constante de temps $\theta$ de son enveloppe nous permet de calculer $\tau_{2}$ grâce à la formule (XIII-13)

$$
\frac{2}{\theta}=\frac{1}{\tau_{1}}+\frac{1}{\tau_{2}}
$$

D - La nutation dans le noir.

Le temps $\tau_{2}$, comme $\tau_{1}$, dépend de I'intensité lumineuse suivant la formule (XII-10). Pour atteindre directement $T_{2}$, on peut observer la nutation dans le noir, comme précédenment pour $\mathrm{T}_{1}$ nous observions la relaxation dans le noir. A cet effet, un dispositif de relais synchronisés permet, à l'instant où l'on ferme le circuit de radiofréquence pour créer $\mathrm{H}_{1}$, de fermer simul tanément l'obturateur pour supprimer l'irradiation Iumineuse. Le signal tombe à zéro; pendant ce temps la population $\beta$ subit dans le noir une variation sinusoidale, de même fréquence $\omega_{1} / 2 \pi$ que précédemment, mais amortie moins vite puisque les photons ne contribuent plus à cet amortissement. Lorsqu'on rétablit la lumière, un instant $\Delta t$ plus tard, l'intensité initiale observée 
représente la population à laquelle avait conduit cette évolution dans le noir. En faisant varier $\Delta t$, on peut ainsi décrire point par point la sinusoide de nutation dans le noir. En pratique, seule nous intéresse son enveloppe; aussi choisissons-nous $\Delta t$ égal à un nombre entier de fois İa période de la sinusoide, de façon à n'obtenir que ses maxima, comme on le voit sur les figures $13 a$ et $13 \mathrm{c}$. Si l'on réalise directement la superposition photographique de ces courbes (figure 13d), on matćrialise l'enveloppe de la sinusoide de nutation dans le noir et on mesure sa constante de temps $\Theta$, d' où l'on tire aussitôt $\mathrm{T}_{2}$ grâce à la formule suivante déduite de (XIII-13) :

$$
\frac{2}{\omega}=\frac{1}{\mathrm{~T}_{1}}+\frac{1}{\mathrm{~T}_{2}}
$$

(Ia comparaison des figures $13 b$ et $13 d$ illustre bien la différence entre les temps $\theta$ et $\Theta)$.

L'interprétation des transitoires de ${ }^{201} \mathrm{Hg}$ sereit assez différente (cf paragraphe XVI) parce que les phénomènes sont beaucoup plus compliqués dans le cas de 4 niveaux. Nous $n$ 'en parlons pas ici puisque nous ne les avons pas encore observés. I'application de cette méthode dynamique à ${ }^{201} \mathrm{Hg}$ est en effet rendue plus difficile par des temps de relaxation beaucoup plus courts. Le temps de fermeture et d'ouverture de l'obturateur photographique inversé, de grand diamètre $(6 \mathrm{~cm})$ actuellement utilisé ne serait peut-être plus assez court par rapport à des temps de relaxation de l'ordre de quelques centièmes de seconde; mais il nous permettrait au moins d'observer avec des déformations l'allure des phénomènes transitoires. Ce qui nous a empêché de les observer jusqu'ici, c'est le fort niveau de bruit à basse fréquence du signal des photomultiplicateurs, probablement dû̀ à notre source lumineuse. Si aucun filtre de fréquence n'élimine certaines composantes de ce bruit, son niveau atteint 3 à $5 \%$ du signal de résonance optique total. Or les variations des signaux observés avec ${ }^{201} \mathrm{Hg}$, lorsque le diamètre de l'obturateur photographique réduit l'ouverture du faisceau lumineux d'irradiation, ne dépassent pas 10\% du signal de résonance optique; le rapport signal/bruit est beaucoup trop faible dens ces conditions pour permettre des mesures. Les longs temps de relaxation de ${ }^{199} \mathrm{Hg}$ nous permettent de gagner sur ce rapport de deux manières : 
a) ils augmentent l'amplitude des signaux óbservés - b) ils permettent l'utilisation à la détection d'un filtre passe-bas éliminant une grande partie du spectre de bruit (en particulier les composantes voisines de $50 \mathrm{c} / \mathrm{s}$ ). Dans la méthode statique, le galvanomètre avec sa constante de temps d'environ 10 secondes joue le rôle d'un filtre ̀̀ très basse fréquence, et permet le tracé de courbes de résonarice avec un rapport signal/bruit acceptable même lorsque le signal ne vaut qu'environ $1 \%$ du signal de résonance optique.

\section{XIV - La méthode de calcul.}

Nous nous proposons maintenant de justifier par le calcul les formules citées dans les deux paragraphes précédents. Des calculs détaillés de forme de raie ont été effectués pour ce type de problème par Brossel (3) et Winter (38). L'un et l'autre utilisent les résultats des calculs de Rabi (37) et Majorana (39) sur les probabilités de transition entre sous-niveaux Zeeman dans un champ tournant; mais ceci n'est possible que si l'on suppose l'existence d'une probabilité par unité de temps définie pour que la perturbation de nature aléatoire (relaxation, absorption ou émission d'un photon) arrache Isatome à la superposition cohérente d'états dans laquelle l'a placée le champ tournant. Cette hypothèse n'est compatible qu'avec des durées de vie égales des difrérents sous-niveaux Zeeman; dans le cas de l'état fondamental, traité par Winter, cela signifie une excitation "broad line", dans laquelle les probabilités d'excitation optique $1 / T_{m}$ sont égales pour les différents sous-niveaux $m$. Cette hypothèse n'est plus vérifiée dans le cas d'excitation avec une composante hyperfine sélec-

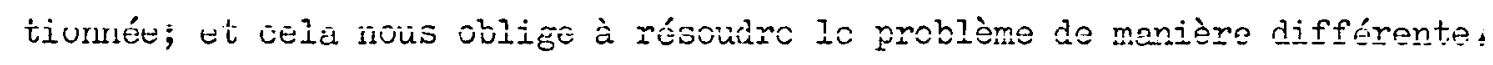

Nous traitons le problème à l'aide du formalisme de la matrice densité $p(t)=\overline{\psi(t)\rangle\left\langle\psi^{*}(t)\right|}$ (la barre représente la moyenne sur tous les atomes), dont les termes diagonaux $\rho_{\mathrm{mm}}$ représentent les populations $X_{\mathrm{m}}$ des sous-niveaux Zeeman. Son évolution au cours du temps est définie par l'équation suivante, que nous allons expliquer : 
(XIV-15)

$$
\frac{d p}{d t}=-i\left[\mathscr{H}_{0}+\mathscr{H}_{1}(t), p\right]+\rho_{R}+\rho_{L}
$$

$H_{0}$ est I'Harniltonien statique : $H_{0}=\gamma_{0} I_{z}$

$H_{1}(t)$ est l'Hamiltonien d'interaction avec le champ $\mathrm{H}_{1}$ tournant à la vitesse $\omega$ :

$$
\mathcal{H}_{1}(t)=\frac{\gamma H_{1}}{2}\left(I_{+} e^{-i \omega t}+I_{-} e^{+i \omega t}\right)
$$

(Remarque : l'assimilation du champ linéaire alternatif de radiofréquence à un champ tournant unique ne pose aucun problème dans notre cas; nous utilisons toujours des amplitudes $\mathrm{H}_{1}$ assez faibles pour que le déplacement relatif de fréquence $\left(\mathrm{H}_{1} / 2 \mathrm{H}_{0}\right)^{2}$ calculé par Bloch et Siegert (40) soit totalement négligeable).

$$
H_{0} \text { et } \mathscr{H}_{1}(t) \text { représentent des interactions auxquelles tous les }
$$
atomes sont également soumis à chaque instant; c'est ce qui permet de calculer leurs effets directement sur la matrice $p(t)$. Hu contraire, les Hamiltoniens $H_{R}(t)$ représentant la relaxation, et $\mathcal{Z}_{L}(t)$ représentant l'irradiation lumineuse sont aléatoires au cours du temps et différents d'un atome à l'autre; il faut donc calculer séparément les termes correspondant à chaque atome, puis faire leur moyenne pour obtenir les matrices $\rho_{\mathrm{R}}$ et $\rho_{\mathrm{L}}$ représentant leur contribution à l'évolution de $p(t)$.

On suppose associé à la perturbation aléatoire $\mathscr{B}_{R}(t)$ représentant la relaxation un temps de corrélation $\tau_{c}$. La contribution d'un Hamiltonien de ce type à l'évolution de $\rho(t)$ a été calculée dans des cas très généraux (41). Dans I'hypothèse dite du rétrécissement par le mouvement, $f_{0_{R}} \cdot \tau_{c} \ll 1$, la matrice $\rho_{R}$ est calculable à partir des éléments $\rho_{m \eta}$ de la matrice $\rho$ et des éléments de matrice de ${ }^{\prime 0}{ }_{R}$; les éléments $\rho_{R m n}$ de la matrice $\rho_{R}$ sont des cumbinaisons linéaires des éléments $P_{m n}$. En l'absence de renseignements sur la perturbation $\mathscr{H}_{R}(t)$, nous utiliserons le modèle suivant :

$(\mathrm{XIV}-16)$

$$
\left\{\begin{array}{l}
\rho_{\mathrm{Rm}}=\frac{1-\rho_{\mathrm{mm}}}{\mathrm{T}_{1}} \\
\rho_{\mathrm{Rmn}}=-\frac{\rho_{\mathrm{mn}}}{\mathrm{T}_{2}} \quad(\mathrm{~m} \neq \mathrm{n})
\end{array}\right.
$$


On montre que dans le cas d'un système à 2 niveaux ( ${ }^{199} \mathrm{Hg}$ par exemple) ce modèle à 2 constantes $T_{1}$ et $T_{2}$ est le plus général possible. Dans le cas d'un système à plus de 2 niveaux ( ${ }^{201} \mathrm{Hg}$ par exemple), $P_{R}$ a en général une forme beaucoup plus compliquée, avec plus de 2 constantes. Il est même peu probable que le modèle simplifié que nous avons choisi puisse correspondre à une perturbation ${ }^{f} L_{R}(t)$ réelle.

Pour expliciter l'action des photons, il faut faire appel à la théorie quantique des champs (42). On trouve que la matrice $p_{I}$ a la forme gónérale d'une matrice de rejaxation. On peut distinguer en réalité deux processus différents : l'absorption des photons, et la retombće de l'état excité. Du moment que le chemp $\mathrm{H}_{0}$ est assez fort pour que la largeur naturello de la raie soit petite devant la séparation des sous-niveaux Zeeman de l'état excité, la retombée se fait dans des ćtats purs, et n'apporte pas de contribution aux termes non diagonaux de $\rho_{L}$; sa contribution aux termes diagonaux se calcule à l'aide des probabilités de transition optique (cf formule(VI-8)). On obtient par exemple en irradiation $\mathrm{\sigma}^{+}$avec une seule composante hyperfine :

$(X I V-17)$

$$
\left\{\begin{array}{l}
\rho_{L m n}=-\frac{1}{T_{m}} P_{m n}+\sum_{n} \frac{3 \lambda^{2}}{8 \pi \tau} \Phi_{1} \cdot A_{n}^{F, n+1} \cdot A_{m}^{F, n+1} \cdot P_{n n} \\
\rho_{L m n}=-\frac{1}{2}\left(-\frac{1}{T_{m}}+\frac{1}{T_{n}}\right) P_{m n} \quad(m \neq n)
\end{array}\right.
$$

Quand on veut rósoudre le système (XIV-15), on remarque que, eu second membre, seul. le prenier terme dépend explicitement du temps. Si nous passons dans lo référentiel tournant à la vitesse $\omega$ du champ $\mathrm{H}_{1}$, en définissant les nouvelles fonctions d'onde $\psi^{\prime}$ et la nouvelle matrice densité $\rho^{\prime}$ par : $(X I V-18) \quad \psi=e^{-i \omega I_{z} t} \cdot \psi^{\prime}$ et $p=e^{-i \omega I_{z} t} \cdot \rho^{\prime} \cdot e^{+i \omega I_{z} t}$

I'équation (XIV-15) se transforme alors en l'équation (XIV-19) dont le second membre re dépend plus explicitement du temps : 
(XIV-19)

$$
\left\{\begin{array}{l}
\frac{d^{\prime \prime}}{d t}=-i\left[\mathscr{H}^{\prime}, \rho^{\prime}\right]+\rho_{R}^{\prime}+\rho_{I}^{\prime} \\
\text { avec } \mathscr{H}^{\prime}=\left(\gamma H_{0}-\omega\right) I_{z}+\frac{\gamma H_{I}}{2}\left(I_{+}+I_{-}\right)=-\Delta \omega I_{z}+\frac{\omega_{I}}{2}\left(I_{+}+I_{-}\right)
\end{array}\right.
$$

En effet le nouvel Hamiltonien H' ne dópend plus du temps; et les matrices $\rho_{R}^{\prime}$ et $P_{I}^{\prime}$, transformées de $P_{R}$ et $P_{L}$, ne dépendent pas non plus explicitement du temps, puisque la forme particulière des matrices $\rho_{R}$ et $\rho_{L}$ fait que l'on obtiont $\rho_{R}$ et $\rho_{I}^{\prime}$ par simple substitution des nouvelles variables $\rho_{m n}^{\prime}$ à la place des anciennes variables $\rho_{m n}$.

Comme notre móthode de détection, à la différence d'autres méthodes

(5), n'est sensible qu'aux variations des populations $x_{m}=p_{\mathrm{mm}}=\rho_{\mathrm{mm}}^{\prime}$ des sous-niveaux Zeeman, nous n'aurons pas besoin pour interpréter les résultats du calcul de passer à nouveau au référentiel fixe. Et nous avons 1'avantage dans le référenticl tournant, lorsque nous cherchons la solution stationnaire (popilations d'équilibre), d'obtenir un simple système linéeire en ecrivant $\frac{d g^{\prime}}{d t}=0$. La résolution du système différentiel dans le cas général nous permettra de calculer les phénomènes transitoires.

$\mathrm{XV}-\mathrm{Cas} \mathrm{do}^{199} \mathrm{Hg}(\mathrm{spin} 1 / 2)$.

$$
\begin{aligned}
& \text { Nous choisissons dans le référentiel tournant les notations : }
\end{aligned}
$$

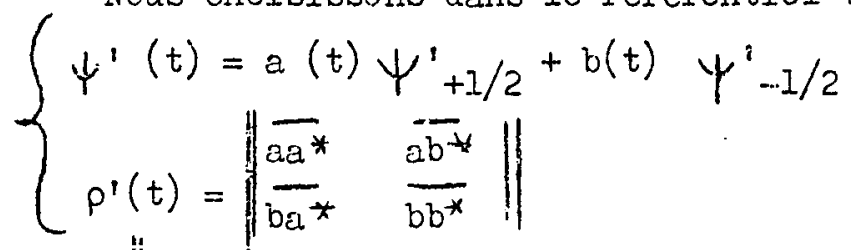

$$
\begin{aligned}
& \text { avec } \mathfrak{H}_{1}=\mid \begin{array}{cc}
-\Delta \omega^{\prime 2}+\omega_{1} / 2 \\
+\omega_{1} / 2+\dot{\omega} \omega / 2
\end{array} \| \text {, on obtient : }
\end{aligned}
$$

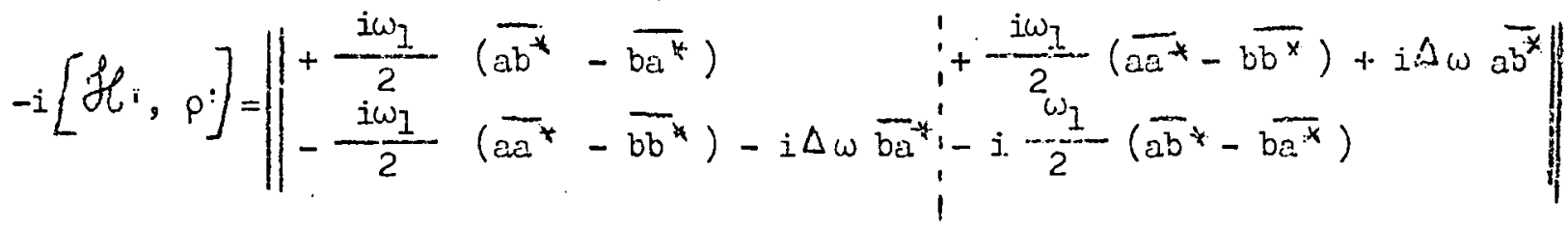


les formules (XIV-16) donnent $\rho_{R}^{\prime}=\left\|\begin{array}{cc}\frac{1-\overline{a a^{*}}}{T_{I}} & -\frac{\overline{a b^{*}}}{T_{2}} \\ -\frac{\frac{1}{b a^{*}}}{T_{2}} & \frac{1-\overline{b b^{*}}}{T_{1}}\end{array}\right\|$

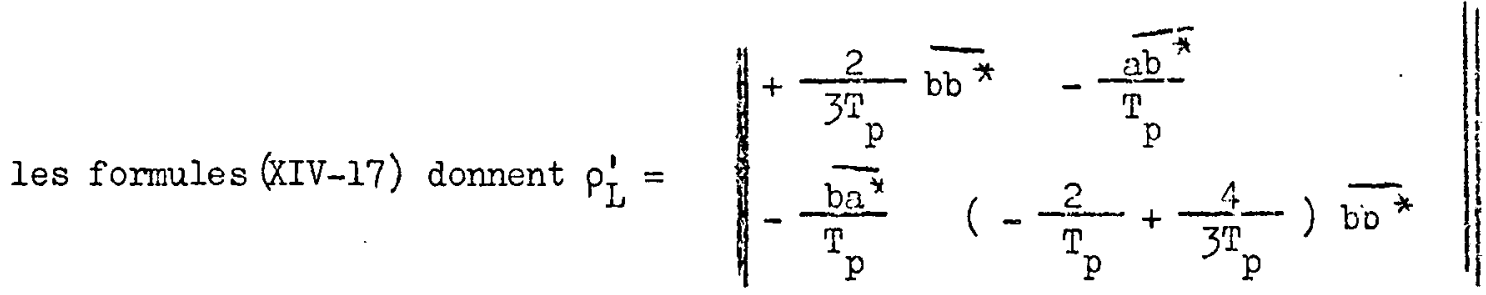
en supposant l'irradiation en $\sigma^{+}$à partir d'une lampe à ${ }^{204} \mathrm{Hg}$ excitant la seu.le composante $199-1 / 2$ et qui n'excite donc pas les atomes du sous-niveau $+1 / 2$ :

$(\mathrm{XV}-20) \quad 1 / \mathrm{T}_{+I / 2}=0$ et donc $\quad 1 / T_{-I / 2}=2 / \mathrm{T}_{\mathrm{p}} \quad$ (d'après $(\mathrm{VI}-4)$ )

Li équation (XIV-19) fournit alors un système de 4 équations différentielles linéaires à 4 fonctions incornues, dont 3 seulement sont indépendentes; elles sont liées par la condition de normalisation des populations :

$$
\alpha+\beta=\overline{a a^{*}}+\overline{b b^{*}}=2
$$

Ce système est grandement simplifié par le choix des nouvelles inconrues : $(X V-21) \quad M=\alpha-\beta=\overline{a a^{*}}-\overline{b b^{*}} ; u=\overline{a b^{*}}+\overline{b a^{*}} ; v=i\left(\overline{a b^{*}}-\overline{b a^{*}}\right)$

Il s'écrit alors :

$(X V-22)\left\{\begin{array}{l}\frac{d M}{d t}=-\frac{1}{\tau_{1} M} \quad+\omega_{1} v+\frac{4}{3_{p}^{m}} \\ \frac{d u}{d t}= \\ \frac{d v}{d t}=-\omega_{1} M \quad-\frac{1}{\tau_{2}} u+\Delta \omega v\end{array} \quad \operatorname{svec}\left\{\begin{array}{l}\frac{1}{\tau_{2}}=\frac{1}{T_{2}}+\frac{1}{T_{p}} \\ \frac{1}{\tau_{1}}=\frac{1}{T_{1}}+\frac{2}{3 T_{p}}\end{array}\right.\right.$

Lu changement près de $\tau_{1}$ en $\mathrm{T}_{1}$ et de $\tau_{2}$ en $\mathrm{T}_{2}$, ce système est identique aux équations macroscopiques de Bloch (34). Mais avec la méthode de détection radio-

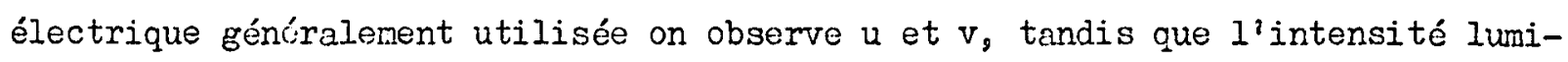


neuse de résonance optique que nous observons est proportionnelle à $\beta=\overline{b^{*}}=1-\frac{M}{2}$.

A. Lá forme de la raie de résonance est donnée par la solution stationnaire de ce système différentiel, dénotée par l'indice $s$ :

$(X V-23)$

$$
M_{s}(\Delta \omega)=M_{0}[I-Z(\Delta \omega)] \text { avec }\left\{\begin{array}{l}
M_{0}=\frac{4 \tau_{1}}{3 T_{p}} \\
Z(\Delta \omega)=\frac{\omega_{1}^{2} \frac{\tau_{1}}{\tau_{2}}}{\Delta \omega^{2}+\frac{1}{\tau_{2}^{2}}+\omega_{1}^{2} \frac{\tau_{1}}{\tau_{2}}}
\end{array}\right.
$$

L'indice 0 sert à dénoter I'état d'orientation obtenu en l'absence de radiofréquence $\left(\omega_{1}=0\right)$; on retrouve bien le résultat du paragraphe VI (tableau I). La différence de population $M_{0}$ croît avec l'intensité lumineuse $1 / T_{p}$ et tend vers 2 (orientation totale) quand $1 / T_{p} \rightarrow \infty$. La forme des courbes de résonance est donnée par $Z(\Delta \omega)$; c'est l'équation d'une courbe de Lorentz. Cela correspond bien aux courbes observées (figure 7). A saturation de la résonance $\left(\omega_{1} \rightarrow \infty\right) Z \rightarrow 1$, c'est-à-dire que la différence de population $\mathbb{M}_{S}$ devient nulle; l'orientation est totalement détruite. De $Z(\Delta \omega)$ on déduit la largeur à mi-hauteur L des courbes de résonance exprimée en fréquence : $(X V-24) \quad L^{2}=\left(\frac{1}{\pi \tau_{2}}\right)^{2}+\left(\frac{x}{\pi}\right)^{2} \frac{\tau_{1}}{\tau_{2}} H_{1}^{2}$

Le carré de la largeux varie linéairement en fonction de $\mathrm{H}_{1}^{2}$; on retrouve bien la formule (XII-12) dans laquelle le coefficient $\mathrm{K}$ est explicité en fonction des deux tèms $\tau_{1}$ et $\tau_{2}$ définis par les formules (XII-10) et (XII-II) que nous avons retrouvées en $(X V-22)$. Nous avons déjà dit comnent le tracé de Ia "droite des largeurs" nous permet de mesurer expérimentalement le "temps de cohérence" ' $\tau_{2}$; on vérifie bien qu'il est conditionné pour une part par 1 'absorption des photons, et qu'il est une fonction linéaire de l'intensité lumineuse. 
L'amplitude relative de la résonance en son centre (divisée par l'amplitude à saturation de la résonance) est $Z(0)$. On vérifie qne : $(\mathrm{XV}-25) \quad \mathrm{Z}(0) \cdot \mathrm{L}^{2}=\frac{\omega_{1}^{2}}{\pi^{2}} \cdot \frac{\tau_{1}}{\tau_{2}}=\left(\frac{x}{\pi}\right)^{2} \frac{\tau_{1}}{\tau_{2}} \mathrm{H}_{\frac{1}{3}}^{2}$

Le produit $\mathrm{Z}(0) \cdot \mathrm{L}^{2}$ est donc représenté en fonction de $\mathrm{H}_{1}^{2}$ par une droite parallèle à la "droite des largeurs"' et passant par l'origine. Cette propriété est bien vérifiée expérimentalement (cf figure 8; droite $\mathrm{n}^{0} 2$ ). On a là un moyen très simple de tester la régularité d'un réseau de courbes et d'anéliorer l'extrapolation de la largeur limite $1 / \pi \tau_{2}$.

\section{B. Les transitoires du pompage optique en l'absence de radio=}

fréquence sont calculés en faisant $\omega_{1}=0$ dans la première équation du système $(\mathrm{XV}-22)$. Ils représentent l'action de la lumière pompante sur les atomes à partir d'un état d'orientation initial $\mathrm{M}_{i}$ arbitraire : $(X V-26) \quad M=M_{0}+\left(M_{i}-M_{0}\right) e^{-t / \tau_{I}}$.

On doit observer une exponentielle dont $l_{2}$ constante de terns est le temps $\tau_{1}$ défini en (XII-11). Ce résultat justifie les affirmations du paragraphe XIII- $h_{\text {; }}$ il aurait pu aussi bien être déduit des formules du paragraphe VI.

C. Les transitoires à résonance $(\Delta \omega=0)$ sont plus faciles à calculer que dans le cas gúnéral parce que le système $(X V-22)$ se réduit aux 2 équations en dv/dt ot dv/dt. Au contraire de Torrey (36), nous éliminons v; cola nous conduit ऐ̀ l'équation différentielle du second orchre à coefficients constants :

$(X V-27) \quad \frac{d^{2} M}{d t^{2}}+\left(\frac{1}{\tau_{1}}+\frac{1}{\tau_{2}}\right) \frac{d M}{d t}+\left(\omega_{1}^{2}+\frac{1}{\tau_{1} \tau_{2}}\right) M=\frac{4}{3 \tau_{2} T_{p}}$ Si $\omega_{1}<\frac{1}{2}\left(\frac{1}{\tau_{2}}-\frac{1}{\tau_{1}}\right)$ la solution est une somme de deux exponentielles, 
qui fait passer la différence de population de $\mathbb{M}_{0}$ à $M_{S}(0) \simeq M_{0}$ puisque $\omega_{1}$ est très petit. Ce cas ne présente donc pas d'intérêt expérimentalement. Si $\omega_{1}>\frac{1}{2}\left(\frac{1}{\tau_{2}}-\frac{1}{\tau_{1}}\right)$ les solutions de l'équation caractéristiques sont : $-\frac{1}{2}\left(\frac{1}{\tau_{2}}+\frac{1}{\tau_{1}}\right) \pm i \sqrt{\omega_{1}^{2}-\frac{1}{4}\left(\frac{1}{\tau_{2}}-\frac{1}{\tau_{1}}\right)^{2}}$

La solution est le produit d'une exponentielle et d'un terme périodique. Dens tous les cas où le terme périodique varie assez vite pour être expérimentalement observable avant d'être complètement anorti, c'est-à-dire si $\omega_{1} \gg \frac{1}{2}\left(\frac{1}{\tau_{2}}-\frac{1}{\tau_{1}}\right)$, on peut négliger le deuxième terme sous le radical; et on obtient la solution :

$(x V-28) \quad M=M_{S}(0)+\left[M_{0}-M_{s}(0)\right] e^{-\frac{1}{2}\left(\frac{1}{\tau_{1}}+\frac{1}{\tau_{2}}\right) t[}\left[\cos \omega_{1} t+\frac{\frac{1}{\tau_{1}}+\frac{1}{\tau_{2}}}{2 \omega_{1}} \sin \omega_{1} t\right]$

On doit observer une sinusoide de fréquence $\mathrm{\gamma H}_{1} / 2 \pi$, amortie exponentiellement avec la constante de temps $\theta$, dont nous avons déjà donné la valeur : (XIII-13) $\quad \frac{2}{\theta}=\frac{1}{\tau_{2}}+\frac{1}{\tau_{1}}$

Nous avons vérifié que la fréquence de la sinusoide est bien proportionnelle à $\mathrm{H}_{1}$ et que son enveloppe exponentielle en est indépendante (cf paragraphe XIII-C).

D, Les transitoires dans le cas général, hors résonance, s'obtiennent en résolvant le systène différentiel complet. L'élimination de $u$ et $v$ conduit à l'équation différentielle du Jème ordre :

$(x V-29)$

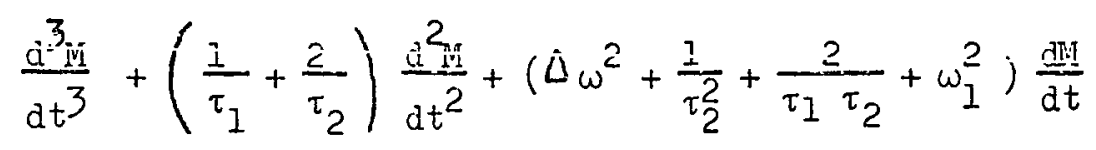

$$
\begin{aligned}
& +\frac{1}{\tau_{1}}\left(\Delta \omega^{2}+\frac{1}{\tau_{2}^{2}}+\omega_{1}^{2} \frac{\tau_{1}}{\tau_{2}}\right) M=\frac{4}{3 ! p}\left(\Delta \omega^{2}+\frac{1}{\tau_{2}^{2}}\right)
\end{aligned}
$$


son équation caractéristique peut s'écrire :

$(\mathrm{XV}-30) \quad\left[\mathrm{x}^{2}+\left(\frac{1}{\tau_{1}}+\frac{1}{\tau_{2}}\right) \mathrm{x}+\Delta \omega^{2}+\frac{1}{\tau_{1} \tau_{2}}+\omega_{1}^{2}\right] \cdot\left(\mathrm{x}+\frac{1}{\tau_{2}}\right)=\Delta \omega^{2}\left(\frac{1}{\tau_{2}}-\frac{1}{\tau_{1}}\right)$

Si l'on suppose encore $\omega_{1} \gg \frac{1}{2}\left(\frac{1}{\tau_{2}}-\frac{1}{\tau_{1}}\right)$, on peut calculer des solutions approchées à partir des solutions exactes que l'on connaît dans le cas particulier $\tau_{1}=\tau_{2}$ :

$$
\left\{\begin{array}{l}
-q=-\frac{1}{\tau_{2}}+\left(\frac{1}{\tau_{2}}-\frac{1}{\sigma_{1}}\right) \frac{\Delta \omega^{2}}{\Delta \omega^{2}+\omega_{1}^{2}} \\
-\mu \pm i \omega=-\frac{1}{2}\left(\frac{1}{\tau_{2}}+\frac{1}{\tau_{1}}\right)-\frac{1}{2}\left(\frac{1}{\tau_{2}}-\frac{1}{\tau_{1}}\right) \frac{\Delta \omega^{2}}{\Delta \omega^{2}+\omega_{1}^{2}} \pm i \sqrt{\Delta \omega^{2}+\omega_{1}^{2}}
\end{array}\right.
$$

$$
\begin{aligned}
& M=M_{s}(\Delta \omega)+\left[M_{0}-M_{s}(\Delta \omega)\right]\left\{e^{-k t}\left[(1-c) \cos \bar{\omega} t+\frac{k+\left(q-k_{1}\right) C}{\bar{\omega}} \sin \bar{\omega} t\right]\right. \\
& \vdots \\
& \text { avec } C \simeq\left(1-\frac{\tau_{2}}{\tau_{1}}\right) \frac{\Delta \omega^{2} t}{\Delta \omega^{2}+\omega_{1}^{2}}
\end{aligned}
$$

La figure observée est encore une sinusoide amortie; mais sa fréquence de résonance est plus grande qu'au centre de la résonance, à même amplitude de $H_{1}$; son amplitude est moindre, et elle se trouve centrée sur une aütre exponentielle. Nous avons vérifié numériquement ces formules sur la figure ilad, obtenue avec la même amplitude $\mathrm{H}_{1}$ que la figure $12 \mathrm{c}$ (sur cette dernière, ôn mesure $\left.\gamma_{1} \mathrm{H}_{1} / 2 \pi=1 \mathrm{c} / \mathrm{s}\right)$, mais en imposant un écart à la résonance $\Lambda \omega / 2 \pi=12,5 \mathrm{c} / \mathrm{s}$.

\section{XVI - Cas du ${ }^{201} \mathrm{Hg}$ en alignement.}

Le calcul s'effectue de manière analogue au précédent et nous le détaillons moins. Les symétries lićes à l'alignement compensent un peu la complexité due à l'existence de 4 sous-niveaux. Nous choisissons dans le réfórentiel tournant les notations :

$$
\psi^{\prime}(t)=a(t) \psi^{\prime}+3 / 2+b(t) \psi^{\prime}+1 / 2+c(t) \psi^{\prime}-1 / 2+g(t) \psi_{-3 / 2}^{\prime}
$$


Rappelons qu'avec les notations choisies pour les populations au paragraphe $\mathrm{V}$ :

$$
\alpha=\overline{\mathrm{aa}^{*}} ; \beta=\overline{b \mathrm{~b}^{*}} ; \gamma=\overline{\mathrm{cc}} ; \eta=\mathrm{gg}^{*}
$$

L'Hamiltonien s'écrit :

$\mathcal{H}^{\prime}=\left\|\begin{array}{lllc}-3 \Delta \omega / 2 & \omega_{1} \sqrt{3} / 2 & 0 & 0 \\ \omega_{1} \sqrt{3} / 2 & -\Delta \omega / 2 & \omega_{1} & 0 \\ 0 & \omega_{1} & +\Delta \omega / 2 & \omega_{1} \sqrt{3 / 2} \\ 0 & 0 & \omega_{1} \sqrt{3} / 2 & +3 \Delta \omega / 2\end{array}\right\|$

Comme matrice de relaxation, nous avons choisi arbitrairement :

$P_{R}^{\prime}=\left\|\begin{array}{cccc}\left(1-\overline{a^{*}}\right) / T_{1} & -\overline{a b^{*}} / T_{2} & -\overline{a^{*}} / T_{2} & -\overline{a g^{*}} / T_{2} \\ -\overline{b a^{*}} / T_{2} & \left(1-\overline{b b^{*}}\right) / T_{1} & -\overline{b c^{*}} / T_{2} & -\overline{b g^{*}} / T_{2} \\ -\overline{c a^{*}} / T_{2} & -\overline{c b^{*}} / T_{2} & \left(1-\overline{c c^{*}}\right) / T_{1} & -\overline{c g^{*}} / T_{2} \\ -\overline{g a^{*}} / T_{2} & -\overline{g b^{*}} / T_{2} & -\overline{g^{*}} / T_{2} & \left(1-\overline{g g^{*}}\right) / T_{1}\end{array}\right\|$

Il est peu probable qu'une interaction réelle puisse janais conduire à une matrice de ce type. II ne faudra done pas s'étonner si nous rencontrons certains désaccords avec l'expórience (cas de l'orientation, of paragraphe XVII). Mais les conclusions que nous en tirons dans le cas de l'alignement sont bien vérifiées.

Pour la matrice d'irradiation lumineuse, nous devons distinguer deux cas. Si l'on excite la composente $201-5 / 2$ seule avec une lampe à ${ }^{204} \mathrm{Hg}$ :

$$
\left\{\begin{array}{l}
\frac{1}{T_{+3 / 2}}=\frac{1}{T_{-3 / 2}}=\frac{1,1}{T_{p}} \text { et } \frac{1}{T_{+1 / 2}}=\frac{1}{T_{-1 / 2}}=\frac{0,9}{T_{p}} \\
\rho_{\mathrm{R}} 3 / 2,3 / 2=-\frac{1,1}{T_{p}} \overline{a a^{*}}+\frac{1,01 \overline{a a^{*}}+0,24 \overline{b b^{*}}+0,03 \overline{c c^{*}}}{T_{p}} \\
\rho_{R} 1 / 2,1 / 2=-\frac{0,9}{T_{p}} \overline{b b^{*}}+\frac{0,06 \overline{a a^{*}}+0,45 \overline{b b^{*}}+0,18 \overline{c c^{*}}+0,03 \overline{\mathrm{ga}^{*}}}{}
\end{array}\right.
$$

Si l'on excite la composante $201-3 / 2$ seule avec une lampe à ${ }^{198} 8_{\mathrm{Hg}}$ : 


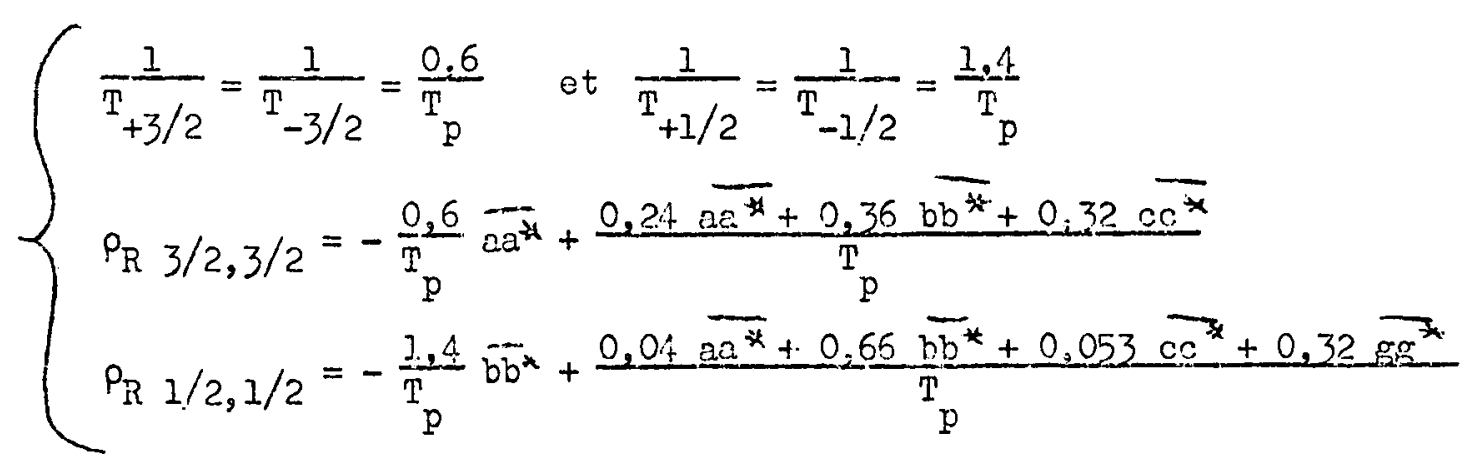

Dans les deux cas on en déduit $P_{R-3 / 2,-3 / 2}$ et $P_{R-1 / 2,-1 / 2}$ par symétrie. En écrivant l'équation (XIV-19) d'évolution de la matrice densité, on obtient un système de 16 équations différentielles. On le simplifie dabord, comne dans le cas de ${ }^{199} \mathrm{Hg}$, en choisissant les nouvelles variables qui sont indiquées ci-dessous. La symétrie des termes d'irradiation lumineuse par rapport aux sous-niveaux $\pm m_{I}$ met en évidence sur ce nouveau système différentiel les symétries suivantes qui réduisert à 5 ie nomore de variables :

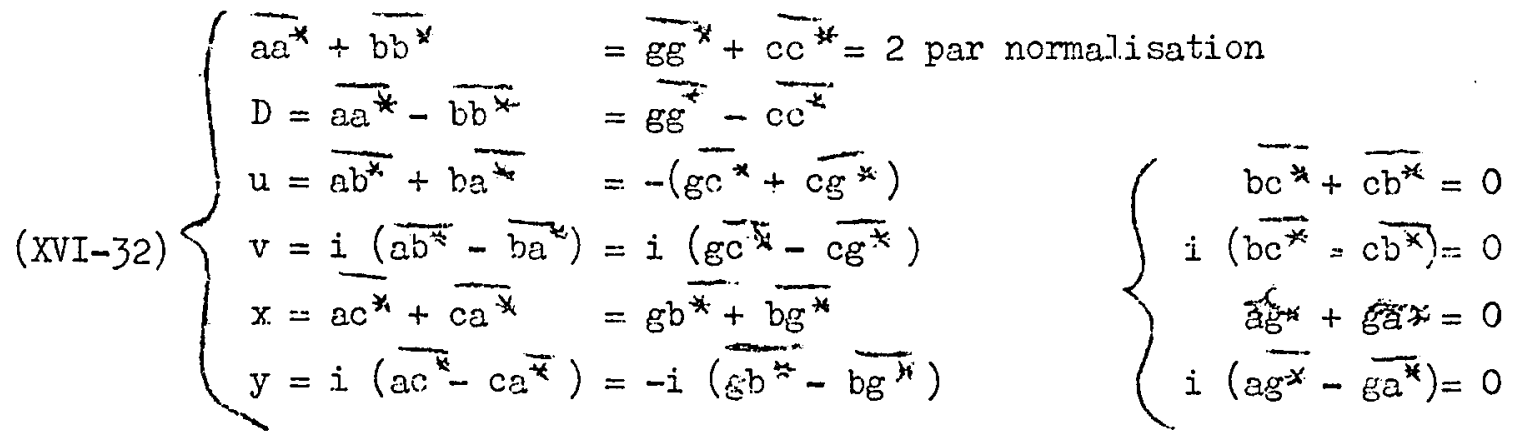

Le système se réduit alors aux 5 équations différentielles :

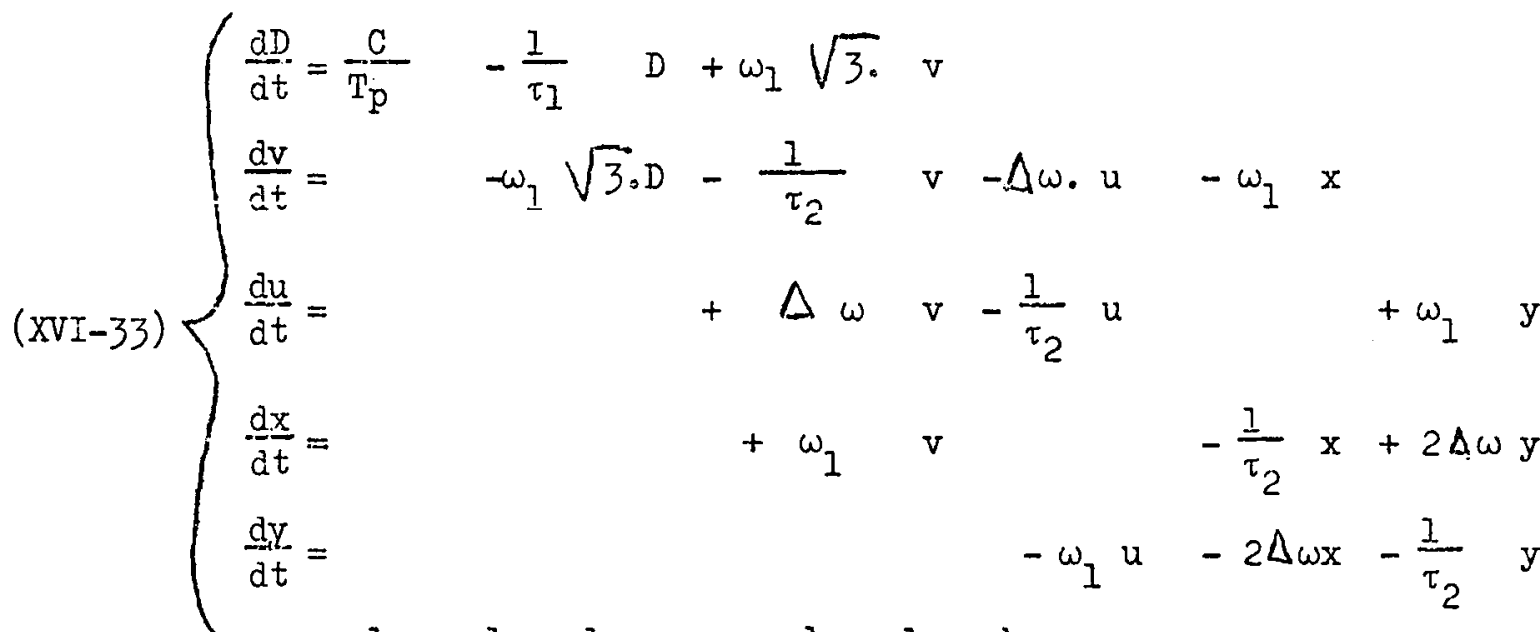

$$
\begin{aligned}
& \text { avec } \frac{1}{\tau_{2}}=\frac{l}{T_{2}}+\frac{l}{T_{p}} \text { et } \frac{1}{\tau_{1}}=\frac{l}{T_{1}}+\frac{k}{T_{p}}
\end{aligned}
$$


Si l'on excite la composante $201-5 / 2$ seule $: \mathrm{k}=0,36 ; \mathrm{C}=0,36$

Si l'on excite la composante $201-3 / 2$ seule : $k=1,04 ; \mathrm{C}=0,64$

A. La solution stationnaire, dénotée par l!indice s, nous donne encore ici la forme de raie. L'élimination de $x, y, u, v$ conduit à :

$(\mathrm{XVI}-34)\left\{\begin{array}{l}\mathrm{D}_{\mathrm{s}}(\Delta \omega)=\mathrm{D}_{\mathrm{o}}[1-\mathrm{Y}(\Delta \omega)] \quad \text { avec } \mathrm{D}_{0}=\mathrm{C} \frac{\tau_{1}}{\mathrm{~T}_{\mathrm{p}}} \text { et }: \\ \mathrm{Y}(\Delta \omega)=\frac{3 \frac{\tau_{1}}{\tau_{2}} \omega_{1}^{2}\left(4 \Delta \omega^{2}+\omega_{1}^{2}+1 / \tau_{2}^{2}\right) \vdots}{\left(4 \Delta \omega^{2}+c_{1} \omega_{2}^{2}+\frac{1}{\tau_{2}^{2}}\right)\left(\Delta \omega^{2}+\omega_{1}^{2}+\frac{1}{\tau_{2}^{2}}\right)+3\left(\frac{\tau_{1}}{\tau_{2}}-1\right) \omega_{1}^{2}\left(c_{i} \Delta \omega^{2}+\omega_{1}^{2}+\frac{1}{\tau_{2}^{2}}\right)}\end{array}\right.$

$D_{0}$ représente la différence de population entre les niveaux $\pm 3 / 2$ et $\pm 1 / 2$ atteinte par le pompage en alignement, en l'absence de radiofréquence; on retrouve bien le résultat du paragraphe VI (tableau II). $D_{0}$ croît avec l'intensité lumineuse $I / T_{p}$; et lorsque celle-ci devient infinie, $D_{0} \rightarrow 1$ si lion excite $l_{a}$ composante $201-5 / 2$, et $D_{0} \rightarrow 0,62$ si l'on excite la composante $201-3 / 2$. Y $(\Delta \omega)$ représente la forme des raies de résonance. Elle ressemble à la formule B obtenue par Brossel (3) dans I'étude de la résonance du niveau excité $6^{3} \mathrm{P}_{1}$ du mercure; mais elle ne s'y réduit que si $\tau_{1}=\tau_{2}$. Il existe une relation linéaire entre leurs inverses:

$(\mathrm{XVI}-35)\left\{\begin{array}{l}B=\frac{3 \omega_{1}^{2}\left(4 \Delta \omega^{2}+\omega_{1}^{2}+1 / \tau_{2}^{2}\right)}{\left(4 \Delta \omega^{2}+4 \omega_{1}^{2}+\frac{1}{\tau_{2}^{2}}\right)\left(\Delta \omega^{2}+\omega_{1}^{2}+\frac{1}{\tau_{2}^{2}}\right)} \\ \frac{1}{Y}=\frac{\tau_{2}}{\tau_{1}} \cdot \frac{1}{B}+1-\frac{\tau_{2}}{\tau_{1}}\end{array}\right.$

On retrouve donc sur $Y(\Delta \omega)$ les deux maxima iatéraũx câlculés sur $D$ lorsque $\omega_{1} \tau_{2}>\frac{1}{2 \sqrt{2}}$. Aux fortes puissances de radiof réquence, lorsque $\omega_{1} \rightarrow \infty$, ces maxima se situent à $\Delta \omega \sim \gamma \mathrm{\gamma H}_{1} / \sqrt{2}$, et $Y(\Delta \omega)$ y prend la valeur 1 , correspondant à l'égalisation des populations. 
Le centre de la résonance par contre ne correspond jamais, même à saturation, à l'égalisation des populations :

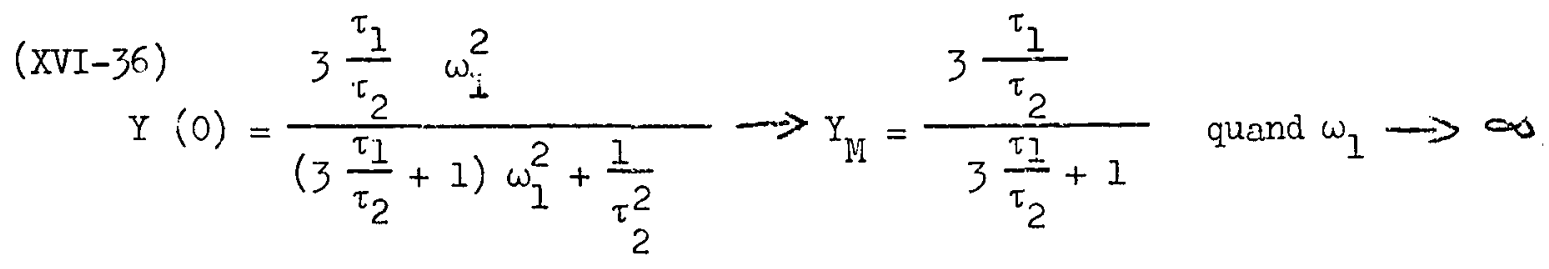

Si l'on excite la composante 201-5/2, on doit s'attendre à avoir $\tau_{1}>\tau_{2}$ et $Y_{M}$ doit être compris entre 0,75 et 1 ; c'est bien ce que nous vérifions expérimentalement. Si l'on excite la composante $201-3 / 2$, et si $\mathrm{T}_{1} \approx \mathrm{T}_{2}$, on doit avoir $\tau_{1} \simeq \tau_{2}$ et $Y_{M}=0,75$ come avec la formule $B$; $c$ ' est bien ce que nous vérifions sur le réseau de courbes de la figure 5. Des formules précédentes, on dóduit encore :

$$
\text { (XVI-37) } \quad \frac{Y_{M}}{Y(0)} \omega_{1}^{2}=\omega_{1}^{2}+\frac{1}{\tau_{2}^{2}\left(1+3 \frac{\tau_{1}}{\tau_{2}}\right)}
$$

c'est-à-dire que, si l'on porte $V_{1}{ }^{2}, Y_{M} / Y(0)$ en fonction de $V_{1}{ }^{2}\left(V_{I}\right.$ est la tension aux bornes du circuit de radiofréquence, proportionnelle à $H_{1}$ ), on doit obtenir une droite de pente 1 . Nous le vérifions expérimentalement (droite $n^{0} 2$ de la figure 9): nous avons lì un moyen de tester la régularj.té d'un réseau de courbes.

La largeur à mi-hauteur. I (exprimée en fréquence) est toujours définie par rapport à l'ordonnée centreile $Y(0)$ sans tenir compte des maxima latéraux. Lorsqu'on cherche I'écart $\Delta \omega_{L}$ pour que $Y\left(\Delta \omega_{L}\right)=Y(0) / 2$, on trouve que $\left(\Delta \omega_{L} \tau_{2}\right)^{2}$ est décrit en fonction de $\left(\omega_{1} \tau_{2}\right)^{2}$ par une branche d'hyperbole très aplatie sur son asymptote. L'équation de cette dernière est :

$$
\left(\Delta_{\omega_{L} \tau_{2}}\right)^{2}=\frac{1}{8}\left[3+\frac{13+15 \tau_{1} / \tau_{2}}{2 \sqrt{10} \tau_{1} \tau_{2}}\right]+\left[\frac{3}{2}\left(1+\frac{\tau_{1}}{\tau_{2}}\right)+\sqrt{10 \frac{\tau_{1}}{\tau_{2}}}\right]\left(\omega_{1} \tau_{2}\right)^{2}
$$

la tangente à l'origine a une équation plus simple :

$$
\left(\Delta \omega_{I} \tau_{2}\right)^{2}=1+\left(2,8+3 \frac{\tau_{1}}{\tau_{2}}\right)\left(\omega_{1} \tau_{2}\right)^{2}
$$


Une étude numérique détaillée à partir de ces équations permet de montrer que l'on peut dans tous les cas remplacer cette hyperbole par une droite avec une très bonne approximation, mêrne aux fortes valeurs de $\omega_{1} \tau_{2}$ : $\left(\Delta \omega_{L \tau_{2}}\right)^{2}=1+\left(3,30+2,80 \frac{\tau_{1}}{\tau_{2}}\right)\left(\omega_{1} \tau_{2}\right)^{2}$ à $2 \%$ près si $1 \leqslant \frac{\tau_{1}}{\tau_{2}} \leqslant 3$ $(\mathrm{XVI}-38)$

$$
L^{2}=\left(\frac{1}{\pi \tau_{2}}\right)^{2}+\left(3,30+2,80 \frac{\tau_{1}}{\tau_{2}}\right)\left(\frac{X}{\pi}\right)^{2} \mathrm{H}_{1}^{2}
$$

On vérifie bien expérimentalement l'existence de cette ''droite des largeurs": (droite $\mathrm{n}^{0} \mathrm{I}$ de la figure 9). Son tracé nous sert systématiquement à obtenir par extrapolation la largeur limite à $\mathrm{H}_{1}$ nul, pour mesurer le temps $\tau_{2}$ qui dans ce cas encore joue le rôle du "tianps de cohérence".

B. Le calcul des transitoires - On peut d'abord, comme dans le cas de ${ }^{199} \mathrm{Hg}$, calculer les transitoires du pompage optique en l'absence de radiofréquence; le calcul est aussi facile, et la solution est encore une exponentielle dont la constante de temps est le temps $\tau_{1}$ de la formule (XII-1I).

Nous ne résoudrons pas le système différentiel dans le cas général, mais seulement dans le cas particulier du centre de la résonance: $\triangle \omega=0$. Le système se réduit alors aux 3 équations en $D,-v$ et $x$; en éliminant ces doux dernières variables on obtient l'équation différentielle di jème ordre à coefficients constants : (XVI-39)

$$
\begin{gathered}
\frac{d^{3} N}{d t^{3}}+\left(\frac{1}{\tau_{1}}+\frac{2}{\tau_{2}}\right) \frac{d^{2} N}{d t 2}+\left(4 \omega_{1}^{2}+\frac{1}{\tau_{2}^{2}}+\frac{2}{\tau_{1} \tau_{2}}\right) \frac{d N}{d t}+\frac{1}{\tau_{1}}\left[\omega_{1}^{2}\left(1+3 \frac{\tau_{1}}{\tau_{2}}\right)+\frac{1}{\tau_{2}^{2}}\right] N \\
=\frac{C}{T_{p}}\left(\omega_{1}^{2}+\frac{1}{\tau_{2}^{2}}\right)
\end{gathered}
$$

I'équation caractéristique peut s'écrixe sous la forme:

$$
(\mathrm{XVI}-40)\left[\mathrm{x}^{2}+\left(\frac{1}{\tau_{1}}+\frac{1}{\tau_{2}}\right) \mathrm{x}+\hat{+} \omega_{1}^{2}+\frac{1}{\tau_{1} \tau_{2}}\right] \cdot\left(\mathrm{x}+\frac{1}{\tau_{2}}\right)=\omega_{1}^{2}\left(\frac{1}{\tau_{2}}-\frac{1}{\tau_{1}}\right)
$$


L'équation peut être résolue exactement quand $\tau_{1}=\tau_{2}$. Si l'on n'est plus dans ce cas particulier, on peut à partir des solutions déjà connues calculer des solutions approchées $-q$ et $-\alpha \pm i \omega$ valables quand $\omega_{1} \gg \frac{1}{\tau_{2}}-\frac{1}{\tau_{1}}$ : $(X V I-4 I)\left\{\begin{array}{l}-q=-\frac{1}{\tau_{2}}+\frac{1}{4}\left(\frac{1}{\tau_{2}}-\frac{1}{\tau_{1}}\right) \\ -H \pm i \bar{\omega}=-\frac{1}{2}\left(\frac{1}{\tau_{I}}+\frac{1}{\tau_{2}}\right)-\frac{1}{8}\left(\frac{1}{\tau_{2}}-\frac{1}{\tau_{1}}\right) \pm 2 i \omega_{1}\end{array}\right.$

On doit donc observer une figure analogue à celle de la figure 12d, obtenue sur ${ }^{199} \mathrm{Hg}$ hors résonance $(\Delta \omega \neq 0)$, c'est-à-dire la somrne d'une sinusoide exponentiellement amortie et a'une autre exponentielle; mais la fréquence de çtte nouvelle sinusoide observée sur ${ }^{20 I_{\text {Hg }}}$ aligné doit être double de celle observée du ${ }^{199} \mathrm{Hg}$, à même valeur de $\mathrm{H}_{I}$. Nous n'avons pas encore pu observer de transitoires sur ${ }^{201}{ }_{\text {Hg }}$ pour vérifier ce point; mais son interprétation est simple. Dans tous les cas, la fréquence de autation des spins est évidemment la même $\omega_{1} / 2 \pi$; c'est-à-dire que, abstraction faite de la relaxation, tous les atomes qui étaient initialement dans le sous-niveau $\mathrm{m}_{\mathrm{I}}$ se trouvent au bout $\mathrm{du}$ temps $\frac{\pi}{\omega}$ dans le sous-niveau $-m_{I}$, et inversement. Dans la vapeur de ${ }^{199} \mathrm{Hg}$ orientée, on en déduit que la grandeur macroscopique observée $M=X_{+1 / 2}-X_{-1 / 2}$ aura changé de signe au bout de ce temps. Dans la vapeur de ${ }^{201} \mathrm{Hg}$ alignée au contraire, la grandeur macroscopique observée $D=X_{+3 / 2}-X_{+1 / 2}=X_{-3 / 2}-X_{-1 / 2}$ aura au bout de ce temps retrouvé sa valeur initiale, à cause de la symétrie de populations entre les sous-niveaux $\pm 3 / 2$ et $\pm I / 2$; $D$ ne peut donc varier qu'à la fréquence double $\omega_{1} / \pi$, où à uno frŕ́quesso bastoritique de $\omega_{1} / \pi$.

\section{X̂II - Cas du 201 ing en orientation.}

Dans le cas de l'orientation toutes les symétries qui nous ont aidé dans les paragraphes précédents disparaissent, et nous nous retrouvons en présence d'un système comprenant effectivement 15 équations différentielles à 15 inconnues, c'est-à-dire, pour la recherche de la solution stationnaire, devant un système Iinéaire de 15 équations à 15 inconnues. La résolution complète de ce système aboutirait à des calculs inextricables. Mais il est possible d'en 
calculer une solution approchće si l'on se place dans la double hypothèse d'une faible intensité lumineuse $\left(I / T_{p} \rightarrow 0\right)$ et d'une faible pulascarce de radiofréquence $\left(\omega_{1} \rightarrow 0\right)$. On peut donc encore calculer la largeur limite des courbes de résonance à radiofréquence et à intensité lumineuse nulle; on la trouve égale à $l / \pi T_{2}$ comme dans le cas de l'alignement.

Or nous avons observé que les largeurs limites des courbes de résonance, extrapolées à radiofréquence et à intensité lumineuse nulles, sont presque 2 fois pius petites en orientation qu'en alignoment. Dès maintenant cela prouve que nos hypothèses de calcul relatives à la forme de la matrice de relaxation $P_{R}$ ne sont pas bonnes. Sans doute, nos calculs dans le cas de l'alignement semblent en bon accord avec I'expérience; mais reportons-nous à la matrice de relaxation $\rho_{\mathrm{R}}^{\prime}$ du paragraphe XVI : dans le cas de $I^{i}$ alignement tous les termes de la diagonale non principale sont nuls à cause des symétries (cf formules (XVI-32)) et donc les temps de relaxation qui leur correspondent n'entrent pas en jeu dans le calcul, Il suffirait par exemple pour expliquer la différence observée entre les largeurs de raie en orientation et en alignement que le temps de relaxation $\mathrm{T}_{2}$ correspondant aux termes $\overrightarrow{\mathrm{bc}^{*}}$ et $\overrightarrow{\mathrm{cb}^{*}}$ (cest-à-dire à la résonance $+1 / 2 \longleftrightarrow-1 / 2$ ) soit plus long que les autres temps $\mathrm{T}_{2}$ de $l_{\mathrm{a}}$ matrice. Nous nous en tiendrons là pour I'instant; mais nous reviendrons sur ce sujet au chapitre suivant quand nous aurons déterminé I'origine de la relaxation de ${ }^{201} \mathrm{Hg}$. 


\section{- $64-$ \\ Q UATRIEME C H A P I T R E}

ORIGINE DE LA RELAXATION

\section{XVIII - Quelques contrôles des mesures de temps de relaxation.}

Nous avons exposé au début du chapitre précédent (paragraphes XII et XIII) les deux méthodes employées pour la détermination des temps de relaxation $\mathrm{T}_{1}$ et $\mathrm{T}_{2}$. Nous avons dit que dans le cas du ${ }^{201} \mathrm{Hg}$ il nous avait été possible jusqu'ici d'utiliser seulement la méthode statique (largeurs de raies). Nous avons déjà signalé tous les détails de cette méthode très classique, les quelques contrôles auxquels nous l'avons soumise, et la précision d'environ $10 \%$ que la double extrapolation (à $\mathrm{H}_{1}$ nul, et à intensité lumineuse nulle) permet d'atteindre pour les mesures de $T_{2}$; tandis que pour $T_{1}$ nous ne pouvons attendre de cette méthode qu'un ordre de grandeur.

\section{A. La comparaison des résultats obtenus par les deux méthodes est} possible au contraire dans le cas du ${ }^{199} \mathrm{Hg}$ puisque l'on peut, outre la méthode statique, lui appliquer la méthode dynamique (observation des transitoires). Cette seconde méthode est beaucoup plus rapide et permet diobtenir des valeurs de $\mathrm{T}_{1}$ aussi bien que de $\mathrm{T}_{2}$, reproductibles à environ $5 \%$ près; c'est donc elle que nous avons systématiquement utilisée pour ${ }^{199} \mathrm{Hg}$. Nous avons cependant utilisé de nombreuses fois la méthode statique pour comparer les résultats obtenus avec les deux méthodes dans les mêmes conditions; nous faisons cette comparaison directement sur les temps $\tau_{2}$ et $\tau_{1}$ (cf formules XII-10 et XII-1I) sans utiliser l'extrapolation à lumière nulle (les $\tau_{1}$ sont calculés dans la méthode statique d'après la valeur du rapport $\tau_{1} / \tau_{2}$ déduite de la pente de la i iàroite des largeurs'", cf formule XV-24).

En première approximation, c'est-à-dire à 10 ou $20 \%$ près, les deux méthodes donnent des résultats cohérents. L'accord observé entre les $\tau_{I}$ est aussi bon que le permet la précision de nos mesures. Mais les écarts de 10 à $20 \%$ 
observés entre les $\tau_{2}$ sont systématiquement dans le même sens $\left(\tau_{2}\right.$ statique $>\tau_{2}$ dynamique); et cette différence systématique semble supérieure à nos erreurs expérimentales. Si cela est vrai, nous n'en avons pas actuellement d'explication. Rappelons aussi que nous avons toujours observé un excellent accord entre les formes des transitoires et les équations du paragraphe XV; en particulier nous avons plusieurs fois vérifié la fixité de l'enveloppe de la sinusoide de nutation, à résonance, Iorsque sa fréquence varie entre $1 \mathrm{c} / \mathrm{s}$ et $100 \mathrm{c} / \mathrm{s}$. Ceci élimine totalement la possibilité d'existence dans le circuit du photomultiplicateur de capacités parasites trop fortes, qui constitueraient un filtre à trop basse fréquence, et amortiraient plus rapidement la sinusoide, raccourcissant ainsi la valeur mesurée de $\tau_{2}$,

B. La mesure des variations de $\tau_{1}$ et $\tau_{2}$ en. fonction de l'intensité lumineuse, dans la méthode dynamique, n'a pas besoin d'être utilisée systématiquement comme dans la méthode statique. Nous avons cependant effectué plusieurs fois, à titre de contrôle, des mesures par la méthode dynamique avec variation d'intensité lumineuse. La figure 14 en montre un exemple : les quantités $1 / \pi \tau_{1}$ et $1 / \pi \theta$ sont bien représentées par des droites en fonction de 1 intensité lumineuse; à la limite, à lumière nulle, ces droites passent bien par les points $1 / \pi \mathrm{T}_{1}$ et $1 / \pi(1)$ déterninés par mesure directe dans le noir. Ceci est tout à fait satisfaisent. Mais on peut aussi, à partir de ces deux droites, tracer la droite représentant les variations de $1 / \pi \tau_{2}$, et en déduire le rapport des pentes des deux droites $1 / \pi \tau_{2}$ et $1 / \pi \tau_{1}$. Cette mesure souvent recommencée a toujours fourni un rapport d'environ 2 alors que la valeur théoriquement attendue est $1,5=$ (cf formules XV-22).

Ce rapport théorique de 1,5 est calculé à partir des probabilités de transition optiquo; mais les diverses callses qui seraient susceptibles de les modifier restent impuissantes à expliquer un pareil écart : a) le calcul montre que l'irradiation en lumière mal polarisée contenant une part de $\sigma^{-}$ne changerait pas ce rapport; et une part de lumière $\pi$ ne pourrait que le diminuer b) les probabilités de transition sont modifiées en champ fort par suite du dé- 
couplage IJ dans le niveau excité $6^{3} \mathrm{P}_{1}$. Elles ont été calculées par la néthode d'Inglis (19); pour la valeur de 200 Gauss, que nous n'avons guère dépassée, les changements sont de l'ordre de $2 \%$ - c) une irradiation partielle sur la composante $3 / 2$ pourrait augmenter ce rapport (qui vaudrait 3 en effet dans le cas de la composante $3 / 2$ utilisée seule); mais cette irradiation ne peut avoir lieu (cf figure 1) qu'à partir des faibles quantités des isotopes ${ }^{199} \mathrm{Hg}$ et ${ }^{201} \mathrm{Hg}$ contenues dans la lampe à ${ }^{204} \mathrm{Hg}$; et ces quantités, inférieures à $1 \%$, sont trop faibles pour expliquer l'effet.

Cette différence systématique sur les rapports des pentes, nettement supérieure à nos erreurs expérimentales, n'est pas encore comprise. Niais on remarque que cet écart va dans le même sens ( $\tau_{2}$ trop cours) que l'écart observé sur les $\tau_{2}$ entre la méthode dynamique et la méthode statique; et l'on peut se demander si la méthode dynamique, pour une raison qui nous échappe, ne mesure pas des temps $\theta$ systématiquement un peu plus courts que les temps théoriques. Cela nous a conduit à exercer quelques contrôles supplémentaires, qui n'ont d'ailleurs pas éclairci ce point.

C. De faibles champs altematifs parasites $\Delta H_{0}$ sin $S 2$ t ne peuvent pas perturber les mesures de la méthode statique pourvu que soit vérifiée l'une ou l'autre des deux conditions suivantes : a) $\gamma \Delta \mathrm{H}_{0}\left\langle 1 / \tau_{2}\right.$ ou $\left.\left.b\right) \Omega\right\rangle l / \tau_{2}$. Nous avons effectivement vérifié que le champ de balayage utilisé pour la résonance de protons ( $10 \mathrm{c} / \mathrm{s} ; 10$ milliGauss; cf paragraphe $\mathrm{X}$ ) ne gêne absolument pas nos mesures avec cette méthode ( $\mathrm{La}$ conditionjest vérifiée dans le cas de ${ }^{201} \mathrm{Hg}$, et la condition b dans le cas de ${ }^{199} \mathrm{Hg}$ ). Ce champ de balayage par contre déforme assez fortement les figures des transitoires et doit être suppriné quand on les observe. Aussi avons-nous contrôlé la présence éventuelle de champ alternatifs parasites, qui auraient constitué une sürice d'erreur pour la móthode dynamique.

Pour cela nous envoyans sur un oscillographe par l'intermédiaire d'un préamplificateur la tension recueillie aux bornes d'une bobine dont la surface totale atteint environ $10^{6} \mathrm{~cm}^{2}$. Dans le signal recueilli la seule composante identifiable, et nettement au-dessus du niveau de bruit, correspond à un champ 
à $50 \mathrm{c} / \mathrm{s}$ d'environ 0,3 milliGauss d'amplitude (Il est dû au résidu alternatif de l'alimentation Varian, qui, dans l'usage normal de cette alimentation, se trouve complètement filtré par la self beaucoup plus importante de bobines à noyau de fer). Un champ alternatif d'aussi faible amplitude ne peut pas perturber nos observations.

En effet, nous avons fait des mesures systématiques de $\theta$ en présence d'un champ de balayage $\Delta H_{0} \sin S L t$, dont nous avons fiait varier la fréquonce $\Omega / 2 \pi$ entre $I \mathrm{c} / \mathrm{s}$ et $150 \mathrm{c} / \mathrm{s}$. Et nous avons vérifié que si l'amplitude $\Delta \mathrm{H}_{0}$ de ce champ est assez faible, il n'a pas d'effet sur les transitoires du ${ }^{199}{ }^{\circ}{ }^{\circ}$, en dehors du cas particulier où sa fréquence est voisine de la fréquence de nutation des spins nucléaires autour du charnp tournant $\mathrm{H}_{1}$. Cette condition de 'résonance" $\subseteq=\gamma_{1} \mathrm{H}_{1}$ 'interprète dans le référentiel tournant à la vitesse angulaire $\omega \mathrm{du}$ charp $\mathrm{H}_{1}$, exactement comme la condition $\omega=\gamma \mathrm{H}_{0} \mathrm{~s}^{\prime}$ interprète dans le référentiel fixe : c'est-à-dire que l:on peut dans le référentiel tournant décomposer le champ de balayage en deux champs d'amplitude $\Delta \mathrm{H}_{\mathrm{o}^{\prime}} / 2$ tournant autour de $\mathrm{H}_{1}$ en sens opposés à la vitesse angulaire $\Omega$; si le mouvement de nutation des spins autour de $\mathrm{H}_{1}$ se fait avec la mêrne vitesse de rotation $\gamma \mathrm{H}_{1}=52,1^{\prime}$ un de ces deux charaps tournants $\Delta \mathrm{H}_{\mathrm{O}} / 2$ reste en phase avec les spins nucléaires et peut avoir sur eux une action importante. Les figures $15 \mathrm{a}$ et $15 \mathrm{c}$ montrent des exemples des transitoires de ${ }^{199} \mathrm{Hg}$ ainsi déformés au voisinage de cette 'résonance' : la fréquence de balayage $\Omega / 2 \pi=30 \mathrm{c} / \mathrm{s}$ est portée comme étalon de tenps dans le bas de chaque oscillogramme; on mesure $\gamma_{1} / 2 \pi \simeq 33 \mathrm{c} / \mathrm{s}$, et l'anplitude $\Delta \mathrm{H}_{0} \simeq 8$ milliGauss est assez grande pour que y $\Delta \mathrm{H}_{0} \sim\left|\Omega-\gamma_{I}\right|$. Si l'on crée le champ $\mathrm{H}_{I}$ au moment où le champ de balayage passe par la bonne phase, on observe un effet particulièrement net (figure 15a) : l'mplitude de la sinusoide habituelle se trouve modulée presque sinusoidalement.

$L^{*}$ interprétation détaillée de ces phénonènes est assez complexe; nous en retiendrons seulement que un champ alternatif ne peut pas déformer les transitoires s'is vérifie l'une ou l'autre des deux conditions suivantes : a) $\gamma \emptyset \mathrm{H}_{0} \ll I / \tau_{2}$ 
(c'est-àmire que le transitoire est amorti avant que le champ $\Delta H_{0}$ ait pu avoir un effet notable), ou b) $\gamma \Delta \mathrm{H}_{0} \ll\left|\Omega-\gamma \mathrm{H}_{1}\right|$ (c'est-à-dire que l'on est loin de la 'résonance''). Le champ parasite à $50 \mathrm{c} / \mathrm{s}$ que nous avons décelé vérifie la condition $\underline{b}$, avec les champs $\mathrm{H}_{1}$ que nous utilisons couramment. D'autres champs parasites éventuels vérifient certainement la condition a.

\section{La loi exponentielle d'absorption (nous l'avons déjà signalé au} paragraphe IV), peut faire que les signaux observés ne soient plus proportionnels aux populations des sous-niveaux, et soient ainsi déformés. Les densités de vapeur utilisées sont toujours assez faibles pour que nous puissions admettre que le signal de fluorescence mesuré est proportionnel à la quantité de lumière absorbée dans la cellule, soit I $\sim 1-e^{-k_{0} \ell}$, où $\ell$ mesure l'arête de la cellule et $k_{0}$ est le coefficient d'absorption proportionnel au nombre $N_{0}$ d'atomes par $\mathrm{cm}^{3}$ dans la cellule (cf tableau du paragraphe IV). Dans le cas de l'excitation de ${ }^{199} \mathrm{Hg}$ par sa seule composante $1 / 2$ en $\sigma^{+}$, on celcule : $k_{0}=0,20.10^{-12} \mathrm{~N}_{0} \cdot \beta \mathrm{cm}^{-1}$ (cf paragraphe $\mathrm{V}$; $\beta$ est le population du sous-niveau $\left.m_{I}=-1 / 2\right)$. Nous introduisons le coefficient $K$ sans dimension tel que $k_{0} \ell=K \beta$; $\ell$ t nous domnons ses valeurs dans le tableau ci-dessous en fonction de la température $t$ du queusot de 1 a cellule, pour le cas $\ell=2 \mathrm{~cm}$ où nous étions le plus fréquement placés :

\begin{tabular}{l|cccc}
$t$ & $-30^{\circ} \mathrm{C}$ & $-20^{\circ} \mathrm{C}$ & $-10^{\circ} \mathrm{C}$ & $0^{\circ} \mathrm{C}$ \\
\hline$K(L=2 \mathrm{~cm})$ & 0,07 & 0,25 & 0,84 & 2,5 \\
\hline
\end{tabular}

Le signal observé est $I=1-e^{-K \beta}$; il n'est proportionnel ̀̀ $\beta$ que si $K$ est suffisamment petit; il peut encore être assimilé à une fonction linéaire de $\beta$, quand $K$ est grand, si $\beta$ varie peu autour d'une valeur noyenne, c'est-à-dire par exemple si le taux d'orientation est faibie (population d'équilibre atteinte par le pompage $\beta_{0} \simeq 1$ ). Mais dans le cas de forts taux d'urientation, si la densité de vapeur est importante, les signaux peuvent être très déformés. Cela n'est pas grave pour la méthode statique, car la largeur limite est obtenue en extrapolant les largeurs des courbes de faible amplitude, correspondant à de faibles varia-tions de $\beta$, et qui sont donc peu déformées. 
Mais dans le cas de la méthode dynamique, on nesure les temps de relaxation à l'amplitude $l / e$ des exponentielles observées, c'est-à-dire qu'on utilise la variation totale de $\beta$ entre $\beta_{0}$ et 1 , et les écarts peuvent être importants. La figure $15 \mathrm{~d}$ montre un exemple des déformations observées (température du queusot $\left.t=0^{\circ} \mathrm{C} ; \beta_{0}<0,5\right) ;$ la dissymétrie observée sur la sinusoide de nutation mesure l'écart entre la loi linéaire et la loi exponentielle et est en bon accord avec les coefficients $\mathrm{K}$ calculés; elle correspond au fait que l'intensité I croît avec $\beta$ d'autant moins vite que $\beta$ est plus grend. On voit facilement que les ternps $T_{1}$, $\theta$ et $\left(\Theta\right.$, mesurés sur des exponentielles montant de $I_{0}$ à $I_{1}$ sont légèrement raccourcis, tandis que le temps $\tau_{I}$ mesuré sur une exponentielle descendant de $I_{1}$ à $I_{0}$ est légèrement allongé. Cet effet serait donc dans le bon sens pour expliquer que les $\tau_{2}$ mesurés par la méthode dynamique soient un peu trop courts. Son évaluation exacte n'est pas simple car l'opacité de la vapeur augmente fortement avec $\beta$, et l'intensité lumineuse noyenne, vue par les atomes du sous-niveau $-1 / 2$, baisse quand $\beta$ augmente. Plus exactement $1 / T_{p}$ est égal au rapport du nombre de photons absorbés INI - $e^{-K \beta}$ et du nombre $d^{9}$ atomes absorbants $N_{0} \ell \beta ;$ il varie donc comme $\left(1-e^{-K \beta}\right) / K \beta$. Diautre part, il faut tenir compte du fait que le coefficient d'absorption dépend strictement de la fréquence : cela nous oblige à substituer au coefficient d'absorption $\mathrm{k}_{0}$ le coefficient d'opacité équivalente (49), et cela conduit à une expression plus compliquée à la place de $\left(1-e^{-K \beta}\right)$. Les écarts relatifs finalement calculés ne sont importants qu'aux fortes densités de vapeur; ils atteignent tout juste quelques centièmes pour la température du queusot $t=-20^{\circ} \mathrm{C}$, et à peine $1 \%$ pour $t=-30^{\circ} \mathrm{C}$. Dans les conditions où nous avons normalement travajllé ces écarts sont donc négligeables, comme en témoignent expérimentalement : 1) la parfaite synétrie, à la précision de nos mocuros, dos sinusoides obsemvóes avec $t=-2.0^{\circ} \mathrm{C}$ (figure 15b) - 2) 1'identité des pentes mesurées pour les droites $1 / \pi \tau_{1}$ et $1 / \pi \tau_{2}$ en fonction de 1 intensité lumineuse avec $t=-20^{\circ} \mathrm{C}$ et $t=-30^{\circ} \mathrm{C}$, malgré la variation par un facteur 3 de la densité de vapeur (à de plus fortes températures $t$, nous avons observé un écart croissant entre les pentes des droites représentant $1 / \pi \tau_{2}$ et $1 / \pi \tau_{1}$ en fonction de l'intensité lumineuse). 
Remarque : Dans la discussion précédente, nous n'avons pas tenu compte : a) de la couche de vapeur d'environ 1 ou $2 \mathrm{~mm}$ d'épaisseur, située juste derrière la face irradiée de la cellule, et qui n'est pas observée à la détection - b) du fait que les photons réémis traversent une partie de la vapeur absorbante avant de parvenir à la détection. Ces deux effets deviennent importants aux fortes densités de vapeur, et deviennent même prépondérants lorsque t $70^{\circ} \mathrm{C}$.

\section{XIX - Généralités sur les causes de relaxation.}

Nous désignons ici sous le terme général de relaxation toute interaction aléatoire au cours du temps susceptible de provoquer l'évolution du système des spins nucléaires. A chacun de ces processus est associé un temps de corrélation $\tau_{c}$ dont la définition exacte exige l'introduction des fonctions de corrélation (43), mais dont l'interprétation physique est claire : c'est le temps moyen au bout duquel la perturbation a 'oublié'" la valeur qu'elle avait à l'instant initial. Lvant de faire l'étude détaillée de la relaxation dans le cas particulier de chaque isotope, nous allons passer en revue les divers processus de relaxation possibles.

A. Les collisions sur les parois sont dans le oas des vapeurs alcalines à basse pression la cause essentielle de relaxation; l'orientation électronique des atomes alcalins est détruite à chaque collision sur les parois de verre, à moins que celles-ci ne soient recouvertes de paraffines ou silicones à longues chaînes (44) (35). Dans notre cas, $1^{\prime}$ orientation est purement nucléaire et il n'en est pas de même. liux faibles densités de vapeur, avec lesquelles nous opérons, le libre parcours moyen des atomes de mercure (1 mètre pour la température du queusot $t=-20^{\circ} \mathrm{C}$ ) est bien supérieur aux dimensions des cellules que nous utilisons; les atomes rebondissent donc sur les parois et traversent la cellule de part en part en tous sens; de la vitesse moyenne des atomes, calculée par la théorie cinétique, nous déduisons que leur "temps de vol moyen" entre deux collisions sur les parois est $\tau_{\mathrm{v}} \sim 10^{-4}$ seconde dans une cellule de $2 \mathrm{~cm}$ d'arête. Or les temps de 
relaxation les plus courts que nous ayons observés sont de l'ordre de 0,02 seconde. C'est-à-dire que les atomes de mercure subissent dans tous les cas au moins 200 collisions sur les parois avant d'être désorientés.

Si nous appelons $\mathscr{H}_{\mathrm{P}}(t)$ l'Hamiltonien qui représente l'interaction avec la paroi et $\tau_{s}$ le temps de séjour sur la paroi (ou temps de collision) de 1'atome, le résultat précédent montre que $\left|H_{p}\right| \cdot \tau_{s} \ll 1$. Or le temps de corrélation de la perturbation $\mathcal{H}_{\mathrm{p}}(t)$ est au plus égal à $\tau_{\mathrm{s}}$; ce processus de relaxation vérifie donc bien la condition de rétrécissement par le mouvement. Sur le temps $\tau_{s}$ nous $n^{\prime}$ avons pour l'instant que des renseignements très vagues : a) il ne peut être inférieur au temps de $10^{-12}$ seconde d'une collision élastique; mais nous avons de bonnes raisons de penser que les collisions sur les parois ne sont pas élastiques et qu'il est beaucoup plus long - b) il y a de fortes raisons d'admettre qu'il est beaucoup plus court que le temps de vol $\tau_{\mathrm{v}} \sim 10^{-4}$ seconde.

Si $\tau_{S}$ n'était pas beaucoup plus court que $\tau_{v}$ en effet, le nombre moyen d'atomes adsorbés sur les parois de la cellule serait du même ordre de grandeur ou plus grand que le nombre d'atomes en mouvement dans la vapeur et dont nous observons le signal de résonance optique. Et ceci ne semble pas possible d'après l'expérience suivante : Nous avons chauffé à $300^{\circ} \mathrm{C}$ une cellule remplie de vapeur sèche, ce qui doit provoquer un dégazage au moins partiel de ses parois, et nous n'avons observé aucune variation appréciable du signal de résonance optique, c'est-à-dire du nombre d'atomes en phase vapeur. Ceci est en accord avec l'expérience faite par d'autres expérimentateurs avec chauffage jusqu'à $700^{\circ} \mathrm{C}$ (45). Il serait extrêmement curieux, qu'un film d'atomes de mercure adsorbés sur les parois puisse rester en équilibre avec une densité de vapeur constante, lorsque sa température subit une aussi forte variation.

B. Des collisions en phase gazeuse peuvent également se produire, soit sur des impuretés gazeuses, soit entre atomes de mercure (à la température du queusot $t=-20^{\circ} \mathrm{C}$, le libre parcours moyen de 1 mètre indique qu'il y a environ une collision $\mathrm{Hg}-\mathrm{Hg}$ pour 50 collisions sur les parois). Les temps de corrélation correspondants sont de l'ordre de $10^{-12}$ seconde s'il s'agit de collisions élastiques; 
mais on peut envisager la formation de molécules éphømères ayant des durées de vie beaucoup plus longues.

C. Les inhomogénéités spatiales du champ directeur $\mathrm{H}_{0}$ et du champ de radiofréquence $\mathrm{H}_{I}$ sont vues par l'atome en mouvement comme des champs aléatoires $\Delta \overrightarrow{\mathrm{H}}_{0}(t)$ et $\Delta \overrightarrow{\mathrm{H}}_{1}(t)$ dont nous étudierons l'effet au paragraphe suivant. Disons tout de suite que ces perturbations aléatoires vérifient la condition de rétrécissement par le mouvement, et que leurs effets sont assez faibles pour être en général négligeables.

D. L'effet du queusot. Il y a un échange permanent d'atomes de mercure entre la goutte liquide du queusot et la vapeur située dans la cellule (On calcule la densité de vapeur dans la cellule (23) en écrivant précisément la condition d'équilibre dynamique de cet échange, c'est-à-dire l'égalité du nombre d'atomes de mercure traversant le tube du queusot, venant de la cellule vers la goutte liquide, et du nombre d'atomes le traversant en sens inverse). Et l'on peut penser que les fortes interactions auxquelies sont soumis les atomes dans la goutte liquide ont pour effet de les désorienter aussitôt. La "durée de vie"' d'un atome orienté est donc au plus égale à son temps de séjour dans la cellule $\tau$, entre l'instant où il y pénètre venant de la goutte liquide et l'instant où il se condense à nouveau dans le queusot.

On peut évaluer un ordre de grandeur de ce temps $\tau_{q}$ dans le cas des cellules que nous avons le plus fréquement utilisées, et dont la tête cubique est reliée au queusot par un capillaire d'enviiron $1 \mathrm{~mm}$ de diamètre et $10 \mathrm{~mm}$ de longueur. (figure 16a). Nous pouvons raisonnablement admettre que presque tous les atomes venant de la cellule et ayant franchi le capillaire seront désorientés avant d'y rentrer. Mais dans le franchissement du capillaire beaucoup d'atomes rencontreront ses parois; et puisque nous avons de fortes raisons de penser que les collisions sur les parois sont diffusantes, il est possible à un tel atome de revenir vers l'endroit d'où il venait. Un raisonnement simple, fondé sur des raisons de symétrie, permet de montrer que le nombre d'atomes venant de la cellule et franchissant finalement le capillaire est supérieur au nombre d'atomes venant de la cellule et 
qui, soit franchissent directement le capillaire, soit viennent rencontrer sa paroi dans la moitié inférieure; c'est-à-dire supérieur au nombre d'atomes qui arrivent sur l'embouchure du capillaire à l'intérieur d'un angle solide $\Omega \simeq \pi \alpha^{2}$ (cf figure 16a; l'angle d'ouverture du capillaire $\alpha=0,1$ radian).

La surface totale des parois de la cellule est $\mathrm{S}=24 \mathrm{~cm}^{2}$, la section du capillaire est $s=0,01 \mathrm{~cm}^{2}$; la proportion d'atomes qui au lieu de rebondir sur la paroi traversent le capillaire est dond supérieure $\frac{S}{\mathrm{~S}} \cdot \frac{\Omega}{2 \pi} \sim 2.10^{-6}$. La probabilité pour qu'un atome sorte de la cellule est donc :

$$
\frac{1}{\tau_{\mathrm{q}}}>\frac{1}{\tau_{\mathrm{v}}} \cdot \frac{\mathrm{s}}{\mathrm{s}} \cdot \frac{\Omega}{2 \pi} \sim 0,02 \mathrm{sec}^{-1}
$$

Cet effet est faible; il est totalement négligeable dans le cas de ${ }^{20 l_{\mathrm{Hg}} \text { où les }}$ largeurs de raies valent plusieurs c/s; mais il peut jouer dans le cas de ${ }^{199} \mathrm{Hg}$. Nous avons vérifié expérimentalement l'existence de cet effet en scellant le gueusot de cellules à ${ }^{199} \mathrm{Hg}$ de façon à y enfermer de la vapeur sèche. Nous avons fait l'expérience sur deux cellules : a) une du type courant, dont nous venons de parler (figure 16a) - b) une cellule de taille identique, mais qui est réunie à son queusot par une transition continue en forme d'entonnoir (figure 16b). Nous avons mesuré les variations du temps de relaxation longitudinale $\mathrm{T}_{1}$ au moment $\mathrm{du}$ scellement :

\begin{tabular}{c|c|c|c} 
& avant scellement & après scellement & $\Delta\left(I / \mathrm{T}_{2}\right)=1 / \tau$ \\
\hline cellule a & $\mathrm{T}_{1}=4,0 \mathrm{~s}$ ou $\mathrm{I} / \mathrm{T}_{1}=0,25$ & $\mathrm{~T}_{1}=5,0$ ou $l / \mathrm{T}_{1}=0,20$ & $0,05 \mathrm{sec}^{-1}$ \\
cellule b & $\mathrm{T}_{1}=1,25 \mathrm{~s}$ ou $\mathrm{I} / \mathrm{T}_{1}=0,80$ & $\mathrm{~T}_{1}=3,0$ ou $l / \mathrm{T}_{1}=0,33$ & $0,47 \mathrm{sec}^{-1}$
\end{tabular}

On voit que la probabilité de désorientation $l / T_{1}$ a diminué dans les deux cas, et que l'effet est beaucoup plus important sur la cellule $\underline{b}$, comme il fallait s'y attendre. Pour la cellule a , nous trouvons bien un résultat en accord avec l'ordre de grandeur calculé plus haut. 


\section{XX - Les inhomogénéités spatiales.}

A. Le champ directeur statique $H_{0}$. Nous appellerons plus précisément $\vec{H}_{0}$ la valeur moyenne du champ sur le volume de la cellule; un atome dans son mouvement à travers la cellule est soumis, en plus du champ fixe $\overrightarrow{\mathrm{H}}_{0}$, à un champ $\Delta \overrightarrow{\mathrm{H}}_{\mathrm{O}}(t)$ aléatoire dont la valeur moyenne sur le volume de la cellule est nulle. Il n'est pas simple de calculer le temps de corrélation correspondant, mais il est certainement de l'ordre de grandeur du temps de vol moyen d'une paroi à l'autre $\tau_{v}$. Ce champ $\Delta \vec{H}_{0}(t)$ a des composantes sur les trois axes $x, y, z$ dont le calcul montre qu'elles sont du même ordre de grandeur. Nous avons dit au paragraphe VIII les précautions prises pour les réduire au minimum, et comment nous avons mesuré l'amplitude maximum $\Delta H_{M}$ dans le volume d'une cellule de la composante $\triangle H_{O Z}$ suivant l'axe $z$ qui porte $H_{0}$. Lorsque $H_{0}=200$ gauss (c'est la valeur au voisinage de laquelle nous avons fait nos mesures les plus précises), nous obtenons ainsi pour ces écarts maximum

\begin{tabular}{l|c|c|c|c} 
& $\Delta \mathrm{H}_{\mathrm{M}} / \mathrm{H}_{0}$ & $\Delta \mathrm{H}_{\mathrm{MI}}\left(\mathrm{H}_{\mathrm{O}}=200 \mathrm{G}\right)$ & $\gamma_{199} \cdot \Delta \mathrm{H}_{\mathrm{MI}}=\Delta \omega_{\mathrm{M}}$ & $\Delta \omega_{\mathrm{III}} / 2 \pi$ \\
\hline Cellule de $2 \mathrm{~cm}$ & $3 \cdot 10^{-6}$ & $0,6 \mathrm{mG}$ & $2,9 \mathrm{sec}^{-1}$ & $0,45 \mathrm{c} / \mathrm{s}$ \\
Cellule de $4 \mathrm{~cm}$ & $4.10^{-5}$ & $8 \mathrm{mG}$ & $38 \mathrm{sec}^{-1}$ & $6 \mathrm{c} / \mathrm{s}$
\end{tabular}

(Rappelons que $\left.\gamma_{201} \approx \gamma_{199} / 3\right): \Delta \omega_{\text {M }}$ mesure l'ordre de grandeur maximum des termes du Hamiitonien $\Delta \mathcal{H}_{0}(t)$ qui représente l'action du champ aléatoire $\Delta \vec{H}_{0}(t)$; puisque $\tau_{c} \sim \tau_{v}$, la condition de rétrécissement par le mouvenent $\left|\Delta \mathscr{L}_{J_{0}}(t)\right| \cdot \tau_{c} \ll 1$ est bien vérifiée. Nous en déduisons : a) que l'effet de l'inhomogénéité du champ statique peut effectivement être calculée comme un phénomène de relaxation raccour-

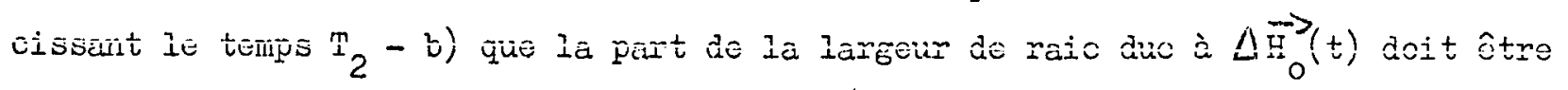
beaucoup plus petite que les valeurs de $\Delta \omega_{N V} / 2 \pi$ indiquées dans le tableau ci-dessus. Cela correspond bien à nos observations, puisque nous mesurons couramment sur ${ }^{199} \mathrm{Hg}$ aussi bien avec de grosses cellules que de petites cellules des largeurs limites $1 / \pi \mathrm{T}_{2}$ de $I^{\prime}$ ordre de $0,3 \mathrm{c} / \mathrm{s}$. 
II est important pour l'interprétation des temps de relaxation mesurés de connaître la valeur de l'élargissement résiduel dû à l'inhomogénéité statique. Son calcul exact exigerait la détermination de la fonction de corrélation du Hamiltonien $\Delta H_{\mathrm{g}}(t)$. Mais on peut raisonnablement admettre que le résultat de ce calcul est semblable aux résultats calculés dans le cas du mouvement Brownien dans un liquide (43) ou dans le cas d'un échantilion tournant (46). Lorsque $\omega \gg l / \tau_{c}$ (et c'est le cas si $\mathrm{H}_{\mathrm{O}} \sim 200$ Gauss) les composantes $\Delta \mathrm{H}_{\mathrm{o}}$ et $\Delta \mathrm{H}_{\mathrm{oy}} \mathrm{n}^{\prime}$ apportent pratiquement aucune contribution à la relaxation, et la contribution due à la composante $\Delta \mathrm{H}_{\mathrm{oz}}$ est :

$$
(\mathrm{XX}-42) \quad\left(I / \mathrm{T}_{2}\right) \sim\left\langle\Delta \omega_{0}^{2}\right\rangle \cdot \tau_{c}=\gamma^{2}\left\langle\Delta \mathrm{H}_{\mathrm{oz}}^{2}\right\rangle \cdot \tau_{\mathrm{c}}
$$

où $\left\langle\Delta \mathrm{H}_{\mathrm{Oz}}^{2}\right\rangle$ représente la moyenne sur le volume de la cellule du carré de la composante $\Delta \mathrm{H}_{\mathrm{Oz}}{ }^{*}$

Pour contrôler 1'application de cette formule, nous avons mesuré, par la méthode dynamique sur une cellule à ${ }^{199} \mathrm{Hg}$ de $4 \mathrm{~cm}$ d'arête, la variation de $\mathrm{T}_{2}$ que l'on provoque en la sournettant à un gradient de champ magnétique connu. Pour observer un effet notable, il faut un gradient de champ assez fort; avec un écart maximum $\Delta \mathrm{H}_{\mathrm{VI}}= \pm 45 \mathrm{mG}$, nous avons observé $\Delta\left(1 / \mathrm{T}_{2}\right)=1,8 \mathrm{sec}^{-1}$. Ciest bien l'ordre de grandeur que fournit la formule $(x X-42)$ en choisissant $\tau_{c}=\tau_{v}=2.10^{-4}$ seconde (cellule de $4 \mathrm{~cm}$ ) et $\sqrt{\left\langle\Delta_{\mathrm{H}}{ }_{\mathrm{Oz}}\right\rangle}=\Delta \mathrm{H}_{\mathrm{M}} / 3$; nous vérifions bien que cette formule est valable pour calculer un ordre de grandeur de l'effet (Une vérification plus détaillée serait possible en créant divers gradients de même configuration géométrique, mais d'amplitudes différentes, et en montrant que $\Delta\left(1 / \mathrm{T}_{2}\right)$ varie proportionnellement à $\left.\Delta \mathrm{H}_{\mathrm{M}}^{2}\right)$.

En définitive, l'application de la formule (XX-42) doit nous permettre de calculer l'ordre de grandeur de la largeur résiduelle d'inhomogénéité dans les conditions normales où nous travailions. Nous trouvons $\Delta\left(1 / \pi \mathrm{T}_{2}\right) \sim 10^{-4} \mathrm{c} / \mathrm{s}$, dans une celıule de $2 \mathrm{~cm}$ d'arête, et $\Delta\left(1 / \pi \mathrm{T}_{2}\right) \sim 10^{-2} \mathrm{c} / \mathrm{s}$ dans une cellule de $4 \mathrm{~cm}$ d'arête. Ces valeurs sont très faibles devant les largeurs limites ( $1 / \pi \mathrm{T}_{2}$ ) couramment mesurées pour ${ }^{199} \mathrm{Hg}$, qui sont de l'ordre de $0,3 \mathrm{c} / \mathrm{s}$. Mais nous avons 
observé jusqu'à $1 / \pi \mathrm{T}_{2}=0,05 \mathrm{c} / \mathrm{s}$; il est alors prudent d'utiliser une petite cellule, dans laquelle la largeur d'inhomogénéi té doit être toujours négligeable.

B. Le champ de radiofréquence $\mathrm{H}_{1}$ peut aussi élargir la raie par son inhomogénéité. On sait en effet que ses changements de direction peuvent causer dans les expériences du jet atomique (47) d'importants déplacements de fréquence atteignant l'ordre de grandeur du Mégacycle/seconde. Soit an effet $\varphi$ l'angle de la direction du champ linéaire alternatif avec une direction de référence perpendiculaire à $\mathrm{H}_{0}$; si le champ alternatif appliqué à un atome en déplacement à travers la cellule subit pendant le temps $\Delta t$ une variation angulaire $\Delta \varphi$ proportionnelle au temps, cela signifie pour le champ toumant correspondant une variation progressive de phase $\Delta \varphi$, équivalente à un changement de sa fréquence de rotation : tout se passe pour l'atome comme s'il était soumis à un champ apparent, tournant à une vitesse angulaire modifiée de $\Delta \omega=\Delta \varphi / \Delta \mathrm{t}$. La valeur élevée des vitesses d'agitation thermique fait que cet effet peut être important même pour de faibles écarts de $\varphi$; en supposant qu'il se produise sur le volume de la cellule un écart $\Delta \varphi=1 / 100$ radian, on calcule un déplacement de fréquence $\Delta \nu=\Delta \omega / \approx \sim 30 \mathrm{c} / \mathrm{s}$. Cet effet, dans notre cas, doit se trouver "moyenné" par le mouvement. Le calcul de l'élargissement résiduel dû à cet effet, compte tenu du rétrécissement par le mouvement, n'a pas encore été fait à notre connaissance, et apparaît comme assez complexe. Nous avons cherché à le déterminer expérimentalement. Pour cela nous avons d'abord vérifié soigneusement l'homogénéité du champ fourni normalement par nos bobines de radiofréquence (cf paragraphe IX) : nous estimons que les écarts relatifs de champ sont de l'ordre de $10^{-3}$ à $10^{-4}$ dans une cellule de $2 \mathrm{~cm}$ d'arête. Puis nous avons rendu ce champ inhomogène en décentrant les bobines : nous $n^{\prime}$ avons observé aucune variation des $T_{2}$ mesurés. Nous avons même inversé les sens des courants dans les deux bobines de radiofréquence, de façon à créer un champ alternatif nul au centre de la cellule, et qui prenne à l'intérieur de la cellule toutes les orientations possibles; nous n'avons encore mesuré aucune variation de $T_{2}$. L'effet résiduel de l'inhornogénéi té du champ de radiofréquence, compte tenu du rétrécissenent par le mouvement, est donc totalement négligeable. 


\section{$X X I$ - Relaxation de ${ }^{201} \mathrm{I}_{\mathrm{H}}$.}

Rappelons d'abord que nous n'avons pu utiliser jusqu'à présent pour ${ }^{201} \mathrm{Hg}$ que la méthode statique, c'est-à-dire que nous n'avons fait de mesures précises que de $\mathrm{T}_{2}$. Sauf indication contraire, il s'agit de mesures en alignement. Les $\mathrm{T}_{2}$ obtenus sont les mêmes que l'irradiation lumineuse se fasse par la composante $201-3 / 2$ avec une lampe à ${ }^{198} \mathrm{Hg}$ ou par la composante $201-5 / 2$ avec une lampe à ${ }^{204} \mathrm{Hg}$; ils semblent à la précision de nos mesures $(\sim 10 \%)$ indépendants de la fréquence. Les $\mathrm{T}_{1}$ évalués indirectement sont dans tous les cas de I'ordre de grandeur de $\mathrm{T}_{2}$.

Le premier fait frappant c'est I'énorme différence entre les temps de relaxation du ${ }^{201} \mathrm{Hg}$ et du ${ }^{199} \mathrm{Hg}$ placés pourtant dans des conditions identiques : Ies $\mathrm{T}_{2} \mathrm{du}{ }^{201} \mathrm{Hg}$ sont en movenne 50 fois plus courts que ceux de ${ }^{199} \mathrm{Hg}$. Un tel écart est impossible à expliquer si l'on suppose que la relaxation est due uniquement à des interactions magnétiques; celles-ci, variant en effet come $\gamma^{2}$, conduiraient au contraire à une relaxation moins forte dans le cas du ${ }^{201} \mathrm{Hg}$, dont le rapport gyromagnétique $\gamma$ est onriron 3 fois plus petit que celui du ${ }^{199} \mathrm{Hg}$. Par contre, le noyau de ${ }^{201} \mathrm{Hg}$, à la différence de celui de ${ }^{199} \mathrm{Hg}$, possède un moment électrique quadrupolaire (17)(48). Nous pensons donc que la cause principale de relaxation du ${ }^{201} \mathrm{Hg}$ est due à ce moment quadrupolaire : dans les collisions d'un atome de ${ }^{20} \mathrm{I} H$ avec la paroi de la cellule, son atmosphère électronique est déformée, et les gradients de champ électrique transitoires ainsi formés peuvent désorienter le noyau par interaction avec son quadrupole.

Nous étions ainsi conduits à penser que les chocs sur les parois, bien qu'ils soient peu efficaces en première approximation (plus de 200 collisions sans désorientation), puissent être cependant la cause essentielle de relaxation. Pour le vérifier, nous avons cherché à modifier le nombre de chocs par unité de temps en utilisant des cellules de différentes tailles. Cette expérience soulève une difficulté : quand on passe d'une cellule à l'autre, on n'est jamais sûr que leurs parois soient de nature parfaitement identique, ni qu'elles contiennent les mêmes 
impuretés (Nous avons remarqué sur une même cellule la variation de $\mathrm{T}_{2}$ lorsque, après un premier usage, on l'ouvre, la dégaze, et la remplit à nouveau de ${ }^{201} \mathrm{Hg}_{\mathrm{Hg}}$ ). Pour nous affranchir de ces effets parasites, nous avons fait la comparaison entre plusieurs cellules de chaque taille:

\begin{tabular}{l|llll|ll} 
arête de la cellule & $4 \mathrm{~cm}$ & $4 \mathrm{~cm}$ & $4 \mathrm{~cm}$ & $4 \mathrm{~cm}$ & $2 \mathrm{~cm}$ & $2 \mathrm{~cm}$ \\
\hline $1 / \pi \mathrm{T}_{2}$ & $8 \mathrm{c} / \mathrm{s}$ & $8 \mathrm{c} / \mathrm{s}$ & $6 \mathrm{c} / \mathrm{s}$ & $7 \mathrm{c} / \mathrm{s}$ & $15 \mathrm{c} / \mathrm{s}$ & $11 \mathrm{c} / \mathrm{s}$ \\
$\mathrm{T}_{2}$ (seconde) & 0,040 & 0,040 & 0,053 & 0,045 & 0,021 & 0,029
\end{tabular}

On vérifie bien que en rnoyenne le temps $\mathrm{T}_{2}$ est environ 2 fois plus faible dans une cellule de dimension double, où les chocs sur les parois sont 2 fois moins fréquents. Hous avons ainsi la certitude que la cause prépondérante de la relaxation $d u{ }^{201} \mathrm{Hg}$ réside dans les chocs sur la paroi. Nous vérifions également que le temps de séjour $\tau_{\mathrm{S}}$ sur la paroi est bien plus petit que le terms de vol moyen $\tau_{\mathrm{v}}$. Si $\tau_{\mathrm{S}}$ était plus long que $\tau_{\mathrm{v}}$ en effet, le changement de dimension de la cellule ne nodifierait guère le temps d'interaction des atomes et de la paroi.

Nous pouvons alors faire un calcul d'ordre de grandeur de cette relaxation quadrupolaire. Lorsqu'un atome rebondit ou se trouve 'adsorbé' sur la paroi durant le temps $\tau_{s}$, il doit être soumis à des interactions beaucoup plus faibles que les interactions couramment mesurées dans les liaisors moléculaires (48)(50). Nous pouvons raisonnablement admettre que le gradient de champ électrique auquel il est soumis est inférieur au gradient créé par une cherge électronique e située à la distance d'un rayon atomique $r\left(\sim 3.10^{-8}\right.$ cr pour le mercure $)$ : $\mathrm{dE} / \mathrm{dr}<2 \mathrm{e} / \mathrm{r}^{3}$. Connaissant $\mathrm{Q} \simeq 0,5 \cdot 10^{-24} \mathrm{~cm}^{2}$, on calcule l'énergie d'interaction quadrupolaire correspondante, exprimée en pulsation :

$$
\Delta \omega_{0}=\frac{e Q}{\not h} \cdot \frac{d E}{d r}<\frac{2 e^{2} Q_{n}}{\not h r} \sim 10^{+7} \sec ^{-1}
$$

Les atomes ne sont soumis à cette interaction que lorsqu'ils rencontrent la paroi, c'est-à-dire pendant une fraction du temps égale à $\tau_{s} / \tau_{v}$; et l'ordre de grandeur du carré moyen de l'énergie d'interaction est donc $\left\langle\Delta \omega_{0}^{2}\right\rangle \sim \Delta \omega_{0}^{2} \cdot \tau_{\mathrm{s}} / \tau_{\mathrm{v}}$. 
Nous avons vu (paragraphe XIX) que cette interaction aléatoire, dont le temps de corrélation est au plus égal à $\tau_{s}$, vérifie la condition du rétrécissoment par le mouvement. Nous pouvons obtenir un ordre de grandeur de la largeur de raie en appliquant la formule de rétrécissement vue au paragraphe précédent (XX-42) :

$$
\frac{1}{T_{2}} \sim\left\langle\Delta \omega_{0}^{2}\right\rangle \cdot \tau_{s} \sim \Delta \omega_{0}^{2} \frac{\tau_{s}^{2}}{\tau_{v}}<\left(\frac{2 e^{2} Q}{\not h r^{3}}\right)^{2} \cdot \frac{\tau_{s}^{2}}{\tau_{v}}
$$

Si l'on suppose que les collisions sur la paroi sont élastiques $\bar{s}_{s} \sim 3.10^{-12}$ seconde, et avec $\tau_{\mathrm{v}} \sim 10^{-4} \mathrm{~s}$, on obtient $1 / \mathrm{T}_{2} \sim 10^{-5} \mathrm{sec}{ }^{-1}$; c'est une valeur beaucoup trop faible par rapport aux valeurs observées. Nous sommes ainsi conduits à penser que les collisions ne sont pas élastiques, et que le temps de séjour sur la paroi $\tau_{s}$ est beaucoup plus long. En remplaçant dans l'équation ci-dessus $\mathrm{T}_{2}$ par la valeur observée $(\sim 0,02 \mathrm{sec}$.$) on en déduit \tau_{s}>10^{-8}$ seconde.

Pour éclaircir les phénomènes de relaxation liés aux parois, nous étions amenés à modifier ces dernières. On peut songer à modifier leur nature par des enduits de paraffine par exemple, conne cela a été réalisé avec succès pour le pompage optique des métaux alcalins (44)(35); mais nous rencontrons ici une difficulté supplémentaire du fait que nos parois doivent rester transparentes à l'ultra-violet. Nous nous sommes donc contentés jusqu'à présent l'augmenter leur température en enfermant la cellule dans un four à fenêtres de quartz (on travaille sur une vapeur sèche en scellant au préalable le queusot). Nous n'avons pas tracé une courbe complète de variation en fonction de la température du four; mais les résultats obtenus sont les suivants sur une cellule de $2 \mathrm{~cm}$ d'arête :

\begin{tabular}{l|lll} 
température & $20^{\circ} \mathrm{C}$ & $270^{\circ} \mathrm{C}$ & $350^{\circ} \mathrm{C}$ \\
\hline $1 / \pi \mathrm{T}_{2}$ & $11 \mathrm{c} / \mathrm{s}$ & $0,9 \mathrm{c} / \mathrm{s}$ & $0,3 \mathrm{c} / \mathrm{s}$ \\
$\mathrm{T}_{2}$ & $0,03 \mathrm{~s}$ & $0,35 \mathrm{~s}$ & $1,0 \mathrm{~s}$
\end{tabular}

Ce résultat vient confirmer nos précédentes déductions; car il semble difficile qu'une relaxation en phase gazeuse puisse expliquer d'aussi grands écarts (cuand on double 
la température absolue $\mathrm{T}$ en effet, les vitesses moyennes des atomes ne sont augmentées d'après la loi en $\sqrt{\mathrm{T}}$ que d'entiron $50 \%$ ). Tandis qu'on imagine aisément que l'élévation de températuro des parois puisse diminuer considérablement le temps de séjour $\tau_{s}$ des atomes sur la paroi; ou bien provoquer un dégazage des parois : des impuretés ioniques éventuelles peuvent avoir une interaction beaucoup plus intense avec les atomes de nercure si elles sont fixées sur les parois, que si elles sont diluées dans tout le volume de la cellule.

Pour en teminer avec la relaxation du ${ }^{201} \mathrm{Hg}$, il nous faut revenir sur la différence déjà signalée (paragraphe XVII) entre les largeurs limites obtenues en alignement et en orientation : elles sont plus petites d'un facteur 2 environ en orientation; et cette différence nous anène à supposer que le temps de relaxation $\mathrm{T}_{2}$ propre à la résonance magnétique $+1 / 2 \longleftrightarrow-1 / 2$, observée en orientation seulement, est plus long que les temps $\mathrm{T}_{2}$ correspondant aux résonances ragnétiques $+3 / 2 \longleftrightarrow+1 / 2$ et $-3 / 2 \longleftrightarrow-1 / 2$. Ceci s'interprète très bien dans l'hypothèse d'une relaxation quadrupolaire. On sait en effet que le déplacement des niveaux d'énergie par interaction quadrupolaire varie conme $\mathrm{m}_{\mathrm{F}}^{2} ;$ la distance énergétique des niveaux $+1 / 2$ et $-1 / 2$ n'est donc pas changée par cette interaction, lors du séjour sur la paroi, alors que les distances $+3 / 2 \longleftrightarrow+1 / 2$ et $-3 / 2 \longleftrightarrow-1 / 2$ sont au contraire modifiées. On conçoit donc que la résonance $+1 / 2 \longleftrightarrow-1 / 2$ soit beaucoup moins perturbée que les deux autres.

En définitive, nous avons acquis la certitude que la relaxation de ${ }^{201} \mathrm{Hg}$ se fait essentiellement par interaction quadrupolaire sur les parois de la cellule. Une étude plus détaillée et plus exacte de cette relaxation devient maintenant possible puisque nous savons à partir de quelles hypothèses érrire la matrice de relaxation. Mais nous nous sommes contentés de défricher le problène et cette étude détaillée est entreprise par ailleurs.' Nous avons également mis en évidence la possibilité de réduire les largeurs $1 / \pi \mathrm{T}_{2}$ du ${ }^{201} \mathrm{Hg}$ à moins de $1 \mathrm{c} / \mathrm{s}$. Cette augmentation considérable des temps de relaxation doit permettre : a) d'observer des taux d'orientation beaucoup plus importants en irradiation $\sigma^{+}-b$ ) d'observer les phénomènes transitoires et d'en tirer des rensẹignements complémentaires sur la

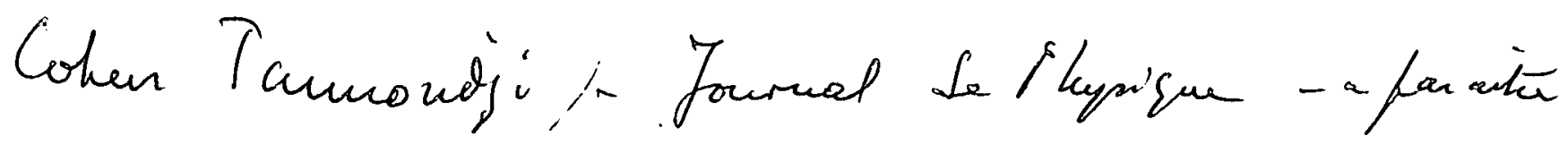


relaxation - c) de mesurer avec une précision accrue le moment nuclćaire de ${ }^{201} \mathrm{Hg}$. XXII - Relaxation de ${ }^{199} \mathrm{Hg}$.

L'étude de la relaxation de ${ }^{201} \mathrm{Hg}$ était relativement facile parce qu'il $\mathrm{y}$ a dans ce cas une cause de relaxation prépondérante sur laquelle nous pouvions faire des tests assez nets sans être gênés par les autres causes de relaxation beaucoup moins importantes. Ceci n'est plus vrai dans le cas du ${ }^{199} \mathrm{Hg}$, dont le noyau ne peut être touché que par son moment magnétique; de plus faibles interactions entrent seules en jeu maintenant, et elles semblent provenir pour une part importante de causes échappant à un contrôle précis. Les temps de relaxation sont en effet extrêmement sensibles aux impuretés, probablement paramagnétiques, présentes dans la cellule : suivant les précautions prises au remplissage de la cellule par ${ }^{199}{ }_{\mathrm{Hg}}$, on a noté sur des cellules identiques de $2 \mathrm{~cm}$ d'arête des temps $\mathrm{T}_{1}$ variant de 2 secondes à 12 secondes et des temps $\mathrm{T}_{2}$ variant de 0,5 seconde à 2 secondes. L'interaction entre ces impuretés et les atomes de mercure se fait certainement en grande partie sur les parois, plutôt qu'en phase gazeuse, comme l'indiquent les deux phénomènes suivants :

a) les très lentes fluctuations des temps de relaxation observées dans une même cellule au cours du temps. Ces fluctuations sont de règle générale dans toutes les cellules et atteignent couramment $30 \%$. Mais nous les avons observées plus précisément sur une cellule particulière : si son queusot est laissé longtemps à température ordinaire, et refroidi seulement à $-20^{\circ} \mathrm{C}$ au moment de la mesure, on trouve $\mathrm{T}_{1}=5,6$ secondes; si maintenant le queusot est laissé en permanence dans I'air liquide et réchauffé à $-20^{\circ} \mathrm{C}$ au moment des mesures seulement, on observe que $\mathrm{T}_{1}$. croît très lentement avec le temps : 2 jours après $\mathrm{T}_{1}=7,5 \mathrm{~s}, 4$ jours après $\mathrm{T}_{1}=9 \mathrm{~s}$ et 8 jours après $\mathrm{T}_{1}$ se stabilise à environ 12 secondes. Il est impossible que des impuretés en phase gazeuse mettent des temps aussi longs à s'établir en équilibre avec le point froid du queusot (La résonance optique en effet permet de vérifier que quelques secondes seulement sont nécessaires à la totalité des atomes 
de mercure pour venir se condenser dans le queusot refroidi à l'air liquide). Mais s'il y a des impuretés adsorbées sur les parois, on conçoit mieux qu'il faille des temps aussi longs pour la décontamination des parois, quand la pression de vapeur de l'impureté est assez basse. Signalons que durant l'évolution de $T_{1}$ sur cette cellule, $\mathrm{T}_{2}$ restait à peu près constant et égal à 2 secondes; les phénomènes qui sont à l'origine de $\mathrm{T}_{2}$ ne sont donc pas tous les mêmes que ceux déterminant $\mathrm{T}_{1}$. b) les temps de relaxation très différents observés sur deur cellules communiquant avec le même queusot et donc remplies avec la même phase vapeur. Nous avions préparé deux cellules communiquantes, de tailles différentes $2 \mathrm{~cm}$ et $4 \mathrm{~cm}$, pour tester l'effet du nombre de collisions par seconde sur les parois, en nous affranchissant des différences de remplissage d'une cellule à l'autre. Les temps observés $\left(\mathrm{T}_{1}=2,2 \mathrm{~s}\right.$. et $\mathrm{T}_{2}=1 \mathrm{~s}$. pour $\mathrm{la}_{\mathrm{a}}$ petite cellule, $\mathrm{T}_{1}=13 \mathrm{~s}$. et $\mathrm{T}_{2}=6 \mathrm{~s}$. pour la grande) varient bien dans le bon sens, mais sont dans un rapport 6 au lieu du rapport 2 attendu (rapport des dimensions linéaires des cellules et par suite des nombres de chocs par seconde dans chacune d'elles). Ceci montre qu'on ne peut pas tester directement dans ce cas l'effet du nombre de collisions par seconde, qui est masqué par des phénomènes du même ordre de grandeur; mais ceci ne peut s'expliquer aussi, puisque les deux cellules contiennent la même phase vapeur, que par une nature différente des parois; nous devons admettre que ces dernières ne contiennent pas normalement les mêmes impuretés, ou ne se contaminent pas de la même façon. (Il est probable aussi que l'effet du queusot est différent d'une cellule à l'autre; mais c'est un effet suffisamment faible pour ne pas changer l'interprétation de ces résultats).

La relaxation sur les parois explique bien également les résultats obtenus en chauffant une cellule de vapeur sèche dans un four à fenêtres de quartz. La courbe (I) de la figure 17 montre les valeurs de $\mathrm{T}_{1}$ ainsi mesurées quand la température du four augmente: $\mathrm{T}_{1}$ part de 2 secondes à $20^{\circ} \mathrm{C}$, ne varie pas beaucoup jusqu'à $100^{\circ} \mathrm{C}$, mais ensuite monte rapidement et atteint plus de 2 minutes à $300^{\circ} \mathrm{C}$. Cette énorme variation (analogue à celle de $\mathrm{T}_{2}$ du ${ }^{201} \mathrm{Hg}$ ) nous conduit à penser que la relaxation longitudinale du ${ }^{199} \mathrm{Hg}$ est conditionnée essentiellement par les parois. 
La courbe (2) de la figure 17 est obtenue en chauffant une cellule de vapeur saturante : nous avons chauffé la partie haute d'une cellule de type courant (figure 16a), en maintenant son queusot dans le thermostat à basse température. Lorsque la température augmente, $\mathrm{T}_{1}$ croît d'abord un peu, mais ensuite il tend vers un maximum de $I$ 'ordre de 20 secondes $\left(I / T_{1}=0,05 \mathrm{sec}^{-1}\right)$ au lieu de continuer à croître suivant la courbe (1). A forte température, la relaxation longitudinale n'est plus déterninée pratiquenent que par l'effet du queusot, qui se trouve ainsi directement mesuré : $T_{I} \simeq \tau_{q}$. On retrouve bien I'orlre de grandeur déjà mesuré pour cet effet (cf paragraphe XIX),

Pendant que l'on mesure sur la vapeur sèche les énormes variations de $\mathrm{T}_{1}$ figurées par la courbo ( 1 ), le temps $\mathrm{T}$ reste à peu près constant entre 0,8 et 1,2 seconde $\left(1 / \pi \mathrm{T}_{2}\right.$ entre 0,40 et $\left.0,26 \mathrm{c} / \mathrm{s}\right)$. Rapprochant ceci de l'observation faite plus haut à propos des fluctuations lentes de $T_{1}$ (paragraphe a), on est tenté de penser que, si la relaxation longitudinale $\left(\mathrm{T}_{1}\right)$ se fait essentiellement sur les parois, la relaxation transversale $\left(\mathrm{T}_{2}\right)$ se fait en grande partie en phase gazeuse. Mais on ne peut tirer de conclusion hâtive; il est possible que le temps $\mathrm{T}_{2}$ soit conditionné aussi par de faibles fluctuations aléatoires du champ $\mathrm{H}_{0}$ ou de la fréquence $\omega / 2 \pi$ du générateur à quartz. En effet, nous ne contrôlons le champ $H_{0}$ avec la résonance du proton qu'à $10^{-6}$ près et il nous est impossible de déceler d'éventuelles fluctuations un peu inférieures à $10^{-6}$; d'autre part, nous avons contrôlé l'oscillateur à quartz à $142 \mathrm{kc} / \mathrm{s}$ en le faisant battre avec un second osciliateur identique : $s^{\prime}$ il est bien stable à $10^{-6}$ près, nous observons des fluctuations supérieures à $10^{-7}$. En définitive des fluctuations de l'ordre de $5.10^{-6}$ sont possibles; si elles se produisent avec un temps de corrélation trop long pour être efficacement moyennées, elles peuvent augmenter les largeurs apparentes d'une quantité $\Delta\left(1 / \pi \mathrm{T}_{2}\right) \sim 0,07 \mathrm{c} / \mathrm{s}$. Cet ordre de grandeur semble tout de même un peu faible pour expliquer les $T_{2}$ observés en. chauffant la vapeur sèche; et cela nous est confirmé par le fait que nous avons avec le même appareillage, à $142 \mathrm{kc} / \mathrm{s}$ également, mesuré sur une autre cellule (de plus grande dimension : $4 \mathrm{~cm}$ d'arête), de façon reproductible à plusieurs jours d'intervalle, des temps $\mathrm{T}_{2}$ 
beaucoup plus longs atteignant 6 et 7 secondes $\left(1 / \pi \mathrm{T}_{2}=0,053\right.$ et $\left.0,045 \mathrm{c} / \mathrm{s}\right)$. Mais pour être bien certains que c'est une cause réelle de relaxation qui limite les $\mathrm{T}_{2}$ quand on chauffe la vapeur sèche, il nous faut attendre d'avoir recommencé la même expérience dans des champs faibles, où la même stabilité relative doit donner des fluctuations totalement négligeables. L'interprétation des $\mathrm{T}_{2}$ reste donc encore incertaine.

L'interprétation exacte des temps de relaxation de ${ }^{199} \mathrm{Hg}$ demande d'autres expériences que nous poursuivons actuellement au laboratoire. Nous pouvons encore signaler les premiers résul.tats de l'étude de la variation de T en fonction de $\mathrm{H}_{0}$, obtenus sur une vapeur saturante (queusot à $-30^{\circ} \mathrm{C}$ ) dans une petite cellule de $2 \mathrm{~cm}$ d'arête du type courant (figure 16a). Ils sont indiqués sur la figure 18 , où nous avons porté $1 / \pi \mathrm{T}_{1}$ en fonction de $\mathrm{H}_{0}$ dans diverses conditions : la courbe (1) est la première courbe tracée sur la cellule, à température ordinaire, avant qu'elle n'ait été chauffée (son queusot était plongé dans l'air liquide plusieurs jours durant, avant l'expérience); la courbe (2) a été obtenue en chauffant la tête de la cellule à $210^{\circ} \mathrm{C}$; la courbe (3) a été obtenue sur la cellule refroidie à température ordinaire, après le chauffage.

La différence entre les courbes (1) et (3) peut s'expliquer soit par le dégazage d'une impureté qui provoque une relaxation en phase gazeuse, soit par une modification de l'état des parois qui augmente leur action désorientante. Nous avons observé systématiquement cette diminution de $\mathrm{T}_{1}$ après chauffage sur toutes les cellules que nous avons chauffées, aussi bien en vapeur sèche qu'en vapeur saturante.

L'asymptote horizontale de la courbe (2) correspond probablement à l'effet du queusot, dont on peut s:attendre à ce qu'il dépende peu de $\mathrm{H}_{0} ; 11$

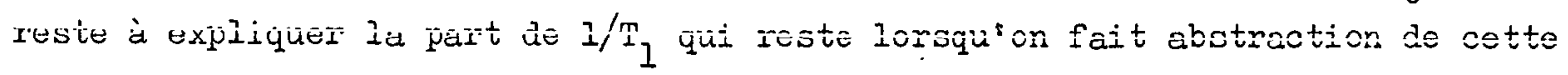
partie constante. On sait que, mojennant certaines hypothèses particulières, on calcule pour les variations de $I / T_{I}$ en fonction de $H_{0}$ des formules du type $\tau_{c} /\left(1+\gamma{ }^{2}{ }_{0}^{2} \tau_{c}^{2}\right)$ (43). On s'attend de manière générale à ce qu'un phénomène aléatoire' de temps de corrélation $\tau_{c} n^{\prime a p p o r t e ~ p l u s ~ q u ' u n e ~ c o n t r i b u t i o n ~ t r e ̀ s ~ f a i b l e ~ a ̀ ~ l a ~}$ 
relaxation Iorsque $\mathrm{\gamma H}_{0} \tau_{c} \gg 1$. La diminution rapide de $1 / \mathrm{T}_{I}$ observée autour de la valeur $H_{0} \sim 200$ Gauss, nous conduit à penser qu'un temps de relaxation $\tau_{c} \sim 10^{-6}$ seconde intervient dans notre cas. Il est possible que nous mesurions ainsi l'ordre de grandeur du temps de séjour $\tau_{s}$ sur la paroi;; ce résultat ne serait pas contradictoire avec l'inégalité $\tau_{s}>10^{-8}$ seconde déduite au paragraphe précédent de l'étude de la relaxation quadrupolaire du ${ }^{201} \mathrm{Hg}$. Tout cela est assez hypothétique; la seule conclusion certaine que nous puissions tirer pour l'instant des courbes de la figure 18 , c'est la grande complexité de la relaxation du ${ }^{199} \mathrm{Hg}$. 
Nous avons, pour la première fois, appliqué avec succès la méthode de pompage optique à des atomes dont l'état fondamental est diamagnétique et qui possèdent un paramagnétisme nucléaire, les isotopes du mercure ${ }^{199} \mathrm{Hg}$ et ${ }^{201} \mathrm{Hg}$, obtenant ainsi une orientation ou un alignement purement nucléaires.

Nous avons obtenu des taux d'orientation importants atteignant $50 \%$, et mis en évidence la possibilité d'en obtenir de plus grands encore en allongeant le temps de relaxation longitudinale $T_{1}$ par chauffage des parois de la cellule qui renferme la vapeur de nercure.

La résonance magnétique nucléaire entre les sous-niveaux Zeenan de l'état fondamental de ${ }^{199} \mathrm{Hg}$ et ${ }^{201} \mathrm{Hg}$ nous a permis de mesurer leurs moments nucléaires. Dans l'ignorance où nous sommes de la valeur précise de la correction diamagnétique, le seul résultat vrainent important est le rapport des moments des deux isotopes, qui ne dépend pas de cette correction :

$$
\mu_{201} / \mu_{199}=1,107416( \pm 5)
$$

Par comparaison avec les mesures de structures hyperfines d'autres auteurs, ce résultat a mis en évidence et permis la mesure précise des anoralies de structure hyperfine du mercure, qui sont supérieures à $1 / 1000$ 。

Nous avons abordé également l'étude des origines de la relaxation des deux isotopes en utilisant à la fois les mesures des largeurs des raies de résonance, et l'observation des phénonènes transitoires du pompage optique et de la relaxation. Cette étude est loin d'être terrinée; mais nous pouvons déjà en tirer quelques conclusions :

a) les collisions sur les parois de silice fondue n'ont qu'une action faibie sur les spinis nucléaires, à la diffórence des spins élentroni ques.

b) les collisions des atomes de mercure sur les parois ne sont pas élastiques; mais l'atone reste sur la paroi après chaque choc pendant un temps au moins 1000 fois supérieur à celui d'une collision élastique. Ce temps diminue fortement si on élève la température des parois. 
c) la relaxation nucléaire de I'isotope ${ }^{201} \mathrm{Hg}$ est due pour l'essentiel à l'interaction des parois avec son moment quadrupaleaire électrique dans les collisions.

Les causes de la relaxation de ${ }^{199} \mathrm{Hg}$ apparaissent comme très complexes; et leur étude se poursuit actuellement. Elle doit nous permettre de préciser en particulier le comportement des atomes sur les parois de la cellule qui renferme la vapeur. C'est là une direction intéressante dans laquelle peut se prolonger le travail que nous avons fait.

Nous espérons bientôt le prolonger également dans une autre direction : l'étude des propriétés nucléaires des isotopes radioactifs du mercure. 


\section{LARGEUR DE RAIE PAR BLLAYAGE HAGNETTQUE}

Nous cherchons à calculer les variations du signal de résonance optique lorsqu'on irradie une cellule de mercure à température ordinaire avec une lampe à mercure dont la raie d'émission est déplacée en fréquence par un champ magnétique (cf paragraphe III).

Le coefficient d'absorption de la cellule en fonction de la fréquence

est :

$$
k_{\nu}=k_{0} e^{-\left(\frac{\nu-\nu_{0}}{u}\right)^{2}}=k_{0} e^{-\omega^{2}}
$$

Nous exprimerons toutes les fréquences dans ce calcul sur l'échelle des nombres sans dimension $\omega$ : l'origine coincide avec la fréquence centrale $\nu_{0}$ de la raie d'absorption de la cellule et l'unité représente la fréquence :

$$
u=\frac{\gamma_{0}}{c} \sqrt{\frac{2 k T}{m}}=\frac{1}{\lambda_{0}} \sqrt{\frac{2 R T}{M}} \simeq 620 \mathrm{Mc} / \mathrm{s}
$$

La largeur Doppler à mi-hauteur est :

$$
\Delta \mathcal{\nu}_{D}=2 \sqrt{\log 2} \cdot \mathrm{u}=1,66 \mathrm{u} \simeq 1030 \mathrm{MC} / \mathrm{s}
$$

Nous supposons que la raie d'émission de la lampe n'est pas 'renversée'', est $\alpha$ fois plus large que la raie d'absorption de la cellule, et qu'elle est déplacée en fréquence d:une quantité X.u; c'est-à-dire que I'intensité émise varie comme $e^{-\left(\frac{\omega-X}{\alpha}\right)^{2}}$.

Nous pouvons supposer, si la densité de vapeur n'est pas trop forte, que l'intensité de résonance optique I est proportionnelle à la quantité de lumière absorbée dans la cellule, soit :

$$
I=\int_{-\infty}^{+\infty} e^{-\left(\frac{\omega-x}{\alpha}\right)^{2}}\left[1-e^{-k_{0} l e^{-\omega^{2}}}\right] d \omega
$$

(cf Pitchell et Zemansky - Resonance radiation and excited atoms - p. 122 et 323 ). 
Si nous travaillons avec de très faibles densités de vapeur, en sorte que $k_{0} l \ll 1,1$ lexpression ci-dessus se simplifie :

$$
I=\int_{-\infty}^{+\infty} e^{-\left(\frac{\omega-X}{\alpha}\right)^{2}} \cdot k_{0} l e^{-\omega^{2}} d \omega=k_{0} l \int_{-\infty}^{+\infty} e^{-\omega^{2}-\left(\frac{\omega-X}{\alpha}\right)^{2}} d \omega
$$

L'exposant de l'exponentielle peut se transformer de la manière suivante :

$$
\omega^{2}+\left(\frac{\omega-x}{\alpha}\right)^{2}=\left(\frac{\sqrt{1+\alpha^{2}}}{\alpha} \omega-\frac{x}{\alpha \sqrt{1+\alpha^{2}}}\right)^{2}+\frac{x^{2}}{1+\alpha^{2}}=y^{2}+\frac{x^{2}}{1+\alpha^{2}}
$$

d' où

$$
\begin{aligned}
& I=k_{0} l \frac{\alpha}{\sqrt{1+\alpha^{2}}} e^{-\frac{x^{2}}{1+\alpha^{2}}} \int_{-\infty}^{+\infty} e^{-y^{2}} d y \\
& I=k_{0} l \sqrt{\pi} \frac{\alpha}{\sqrt{1+\alpha^{2}}} \cdot e^{-\frac{x^{2}}{1+\alpha^{2}}}=I_{0} e^{-\frac{x^{2}}{\beta^{2}}} \text { avec } \beta=\sqrt{1+\alpha^{2}}
\end{aligned}
$$

La courbe expérimentale de balayage magnétique a donc une largeur qui est égale à la largeur de la raie d'absorption multipliée par $\beta=\sqrt{1+\alpha^{2}}$. Inversement, connaissant $\beta$, on en déduit $\alpha=\sqrt{\beta^{2}-1}$.

Application : quand la lampe à mercure est refroidie au maximum, nous lisons sur les courbes expérimentales de la figure 3 une largeur à mi-hauteur d'environ $1900 \mathrm{Mic} / \mathrm{s}$. On en déduit $\beta=1,85$ et $\alpha=1,55$. La largeur à mi-hauteur de la raie diémission de la lampe est donc environ de $1600 \mathrm{Mc} / \mathrm{s}$. 


\section{- $\quad$ N NEXE II -}

\section{CHAMP MLGNETIQUE DE BOBINES D'HEIMHOLTE}

C'est un sujet très classique; mais dans les calculs que nous avons trouvés déjà faits à ce sujet, le paramètre de distance des deux bobines n'intervient pas, ou bien intervient de manière trop compliquée. Cela nous a obligés à refaire les calculs de manière plus utilisable.

$1^{\circ}$ ) Champ créé par une seule bobine mince, de rayon $R$, parcourue par un courant $i$ (Ampères), en un point voisin de son axe $\mathrm{Oz}$, situé à la distance $r$ de cet axe et à la distance $D$ du plan de la bobine. On suppose $r \ll p=\sqrt{R^{2}+D^{2}}$. Composante parallèle à l'axe $\mathrm{Oz}$ de la bobine :

$H_{z}=\frac{i}{10} \cdot \frac{2 \pi R^{2}}{\rho^{3}}\left[1+\frac{3}{4} \cdot \frac{R^{2}-4 D^{2}}{\rho^{2}} \cdot \frac{r^{2}}{\rho^{2}}-\frac{45}{64} \frac{12 R^{2} D^{2}-R^{4}-8 D^{4}}{\rho^{4}} \cdot \frac{r^{4}}{\rho^{4}}+\cdots\right]$

Composante radiale, perpendiculaire à l'axe $\mathrm{Oz}$ : $H_{r}=\frac{i}{10} \frac{3 \pi R^{2}}{\rho^{3}} \cdot \frac{D r}{\rho^{2}}\left[1+\frac{5}{8} \cdot \frac{3 R^{2}-4 D^{2}}{\rho^{2}} \cdot \frac{r^{2}}{\rho^{2}}+\cdots \cdot\right]$

$2^{\circ}$ ) Champ créé par deux bobines minces de même axe, à la distance 2D I'une de l'autre, et de rayon $\mathrm{R}$, en un point voisin de leur centre de symétrie. Le point est repéré par ses coordonnées polaires dans un système dont l'origine est le centre de symétrie : z parallèlement à l'axe, et $r$ perpendiculairement à l'axe. On calcule le champ en ajoutant les expressions que l'on obtient en remplaçant dans les formules ci-dessus $D$ par $z+D$ et $z-D$.

$$
\left\{\begin{array}{l}
H_{z}=\frac{i}{10} \cdot \frac{4 \pi R^{2}}{\rho^{3}}\left[I+\frac{3}{4} \frac{R^{2}-4 D^{2}}{\rho^{2}} \cdot \frac{r^{2}-2 z^{2}}{\rho^{2}}+\frac{15}{64} \cdot \frac{12 R^{2} D^{2}-R^{4}-8 D^{4}}{\rho^{4}} \cdot \frac{24 r^{2} z^{2}-3 r^{4}-8 z^{4}}{\rho^{4}}+\ldots\right] \\
H_{r}=\frac{i}{10} \cdot \frac{4 \pi R^{2}}{\rho^{3}} \cdot \frac{3}{2} \cdot \frac{r z}{\rho^{2}}\left[\frac{R^{2}-4 D^{2}}{\rho^{2}}+\frac{5}{8} \cdot \frac{12 R^{2} D^{2}-R^{4}-8 D^{4}}{\rho^{4}} \cdot \frac{4 z^{2}-3 r^{2}}{\rho^{2}}+\ldots\right]
\end{array}\right.
$$


Dans le cas particulier de deux bobines en position d'Helmholtz $(D=R / 2)$

$$
\left\{\begin{array}{l}
\mathrm{H}_{\mathrm{z}}=\frac{0,900 \cdot n i}{\mathrm{R}}\left[I+\frac{18}{125} \cdot \frac{24 \mathrm{r}^{2} \mathrm{z}^{2}-3 \mathrm{r}^{4}-8 \mathrm{z}^{4}}{\mathrm{R}^{4}}+\ldots\right] \\
\mathrm{H}_{\mathrm{r}}=\frac{0,900 \cdot \mathrm{ni}}{\mathrm{R}} \cdot \frac{72}{125} \cdot \frac{\mathrm{rz}\left(A \mathrm{z}^{2}-3 \mathrm{r}^{2}\right)}{\mathrm{R}^{4}}+\ldots
\end{array}\right.
$$

30) Champ créé par deux bobines épaisses de même axe on un point voisin de leur centre de symétrie. Il faut faire la somme des champs créés par chacune des spires individuelles, c'est-à-dire l'intégrale des formules ci-dessus par rapport aux variables $R$ et $D$ dans la section du bobinage. On introduit pour cela la densité de courant $\sigma$ telle que idn $=\sigma \cdot d R d D$ ou encore $\sigma S=$ ni, où $S$ est la surface de la section du bobinage et $n$ le nombre de spires de chaque bobine (parcourues par le courant $i$ en Ampères). En appelant $R_{0}$ le rayon moyen des bobines on obtient :

$$
\left\{\begin{array}{l}
\frac{R_{0}}{4 \pi n(i / 10)} \cdot H_{z}=\Lambda+\frac{3}{4} B \cdot \frac{r^{2}-2 z^{2}}{R_{0}^{2}}+\frac{15}{64} \text { C } \frac{24 r^{2} z^{2}-3 r^{4}-8 z^{4}}{R_{0}^{4}}+\ldots \\
\frac{R_{0}}{4 \pi n(i / 10)} \cdot H_{r}=+\frac{3}{2} B \frac{2 r}{R_{0}^{2}}+\frac{15}{16} \text { C } \frac{2 r\left(4 z^{2}-3 r^{2}\right)}{R_{0}^{4}}+\ldots
\end{array}\right.
$$

avec les nombres sans dimension :

$$
\left\{\begin{array}{l}
A=\frac{R_{0}}{S} \iint \frac{R^{2}}{\rho^{3}} d R d D \\
B=\frac{R_{0}^{3}}{S} \int \frac{R^{2}\left(R^{2}-4 D^{2}\right)}{\rho^{7}} d R d D \\
C=\frac{R_{0}^{5}}{S} \iint \frac{R^{2}\left(12 R^{2} D^{2}-R^{4}-8 D^{4}\right)}{\rho^{11}} d R d D
\end{array}\right.
$$

-a) Rappelons que pour deux bobines minces en position d'Helmholtz $\left(D=R_{0} / 2\right)$ 


$$
\left\{\begin{array}{l}
A=\frac{8}{5 \sqrt{5}}=0,716 \quad\left(\frac{4 \pi}{10} A=0,900\right) \\
B=0 \\
C=\frac{2^{7} \cdot 3}{5^{4}} A=0,615 A \quad\left(\frac{15}{64} C=\frac{18}{125} A=0,144 A\right)
\end{array}\right.
$$

-b) Intégration exacte pour le cas de bobines épaisses - On appelle a et b les distances au centre de symétrie des deux plans perpendiculaires à l'axe $\mathrm{Oz}$ qui limitent chaque bobine $(a<b)$; on appelle $u$ et $v$ les rayons des deux cylindres d'axe $\mathrm{Oz}$ qui limitent les bobines $(\mathrm{u}<\mathrm{v}$ ) (cf figure). On obtient :

$$
\left\{\begin{array}{l}
A=\frac{R_{0}^{2}}{S}\left(F_{b v}-F_{b u}-F_{a v}+F_{a u}\right) \text { avec } F_{b v}=\frac{b}{2 R_{0}} \log \frac{\sqrt{b^{2}+v^{2}}+v}{\sqrt{b^{2}+v^{2}-v}} \\
B=\frac{R_{0}^{2}}{S}\left(G_{b v}-G_{b u}-G_{a v}+G_{a u}\right) \text { avec } G_{b v}=\frac{R_{0}}{3 b} \cdot \frac{v^{3}}{\left(b^{2}+v^{2}\right)^{3 / 2}}
\end{array}\right.
$$

$\left.4^{\circ}\right)$ Développement approché du coefficient B des termes du second ordre

dans le cas de bobines d'épaisseur moyenne. Nous définissons de nouveaux paramètres sans dimension $k, q$ et $\propto$ supposés petits devant 1 :

rayons limites $\left\{\begin{array}{l}v=R_{0}(1+q) \\ u=R_{0}(1-q)\end{array}\right.$

distances au centre des plans limites $\left\{\begin{array}{l}b=R_{0}\left(\frac{1+\alpha}{2}+k\right) \\ a=R_{0}\left(\frac{1+\alpha}{2}-k\right)\end{array}\right.$

$q$ et $k$ dépendent du format des bobines; $\alpha$ mesure l'écart à la position d'Helmholtz. On calcule le développement de $\mathrm{B}$ en fonction de ces paramètres à partir de l'intégrale exacte indiquée ci-dessus. On simplifie le calcul en notant que par raison de symétrie seuls doivent subsister finalement les termes en $k \cdot q \cdot f\left(k^{2}, q^{2}\right)$; notant encore que $4 \mathrm{qk}=\mathrm{s} / \mathrm{R}_{0}^{2}$, on obtient :

$$
B=\frac{2^{7}}{3.5^{4} \cdot \sqrt{5}}\left(36 k^{2}-31 q^{2}-30 \alpha+\ldots\right)=0,917\left(1,20 k^{2}-1,03 q^{2}-\alpha+\ldots\right)
$$


La condition d'homogénéité s'écrit $30 \alpha \simeq 36 \mathrm{k}^{2}-31 \mathrm{q}^{2}$. Elle indique de combien il faut changer la distance des bobines, suivant leur format, pour annuler les termes du second ordre en $r$ et $z$.

Soit $\alpha_{0}$ la valeur exacte de $\alpha$ qui annule le coefficient $B$; nous poserons $\alpha=\alpha_{0}+\Delta \alpha$. Cela nous permet d'expriner simplement les écarts relatifs de champ par rapport au champ $\mathrm{H}_{\mathrm{O}}$ au centre de symétrie :

$$
\left\{\begin{array}{l}
\frac{\Delta \mathrm{H}_{\mathrm{z}}}{\mathrm{H}_{0}}=+0,96 \Delta \alpha \frac{2 \mathrm{z}^{2}-\mathrm{r}^{2}}{\mathrm{R}_{0}^{2}}+0,144 \frac{24 \mathrm{r}^{2} \mathrm{z}^{2}-3 \mathrm{r}^{4}-8 \mathrm{z}^{4}}{\mathrm{R}_{0}^{4}}+\ldots \\
\frac{\Delta \mathrm{H}_{\mathrm{r}}}{\mathrm{H}_{0}}=+1,92 \Delta \alpha \frac{\mathrm{zr}}{\mathrm{R}_{0}^{2}}+0,576 \frac{\mathrm{zr}\left(4 \mathrm{z}^{2}-3 \mathrm{r}^{2}\right)}{\mathrm{R}_{0}^{4}}+\ldots
\end{array}\right.
$$

$\Delta \alpha>0$ si les deux bobines sont trop écartées $\Delta \alpha<0$ si les deux bobines sont trop rapprochées. C'est la première de ces deux formules qui nous a servi à calculer les écarts théoriques de champ, pour les comparer avec les résultats de nos mesures.

\section{BIBLIOGRAPHIE}

h.E. RU:RK et M.F. PEIERS - J.0.S.h. (1926), 13, 205.

h.F. SAUTER - Zeit fur Physik , (1914), 12.2, 120.

S.T. LIN et t.R. KhUFWhiNN - Rev, of Mod. Phys. (1953), 25, 182.

P. CRIVET et alíi - Ia Résonance Paramagnétique nucléaire (C.N.K.S., Paris 1955), p. 199). 


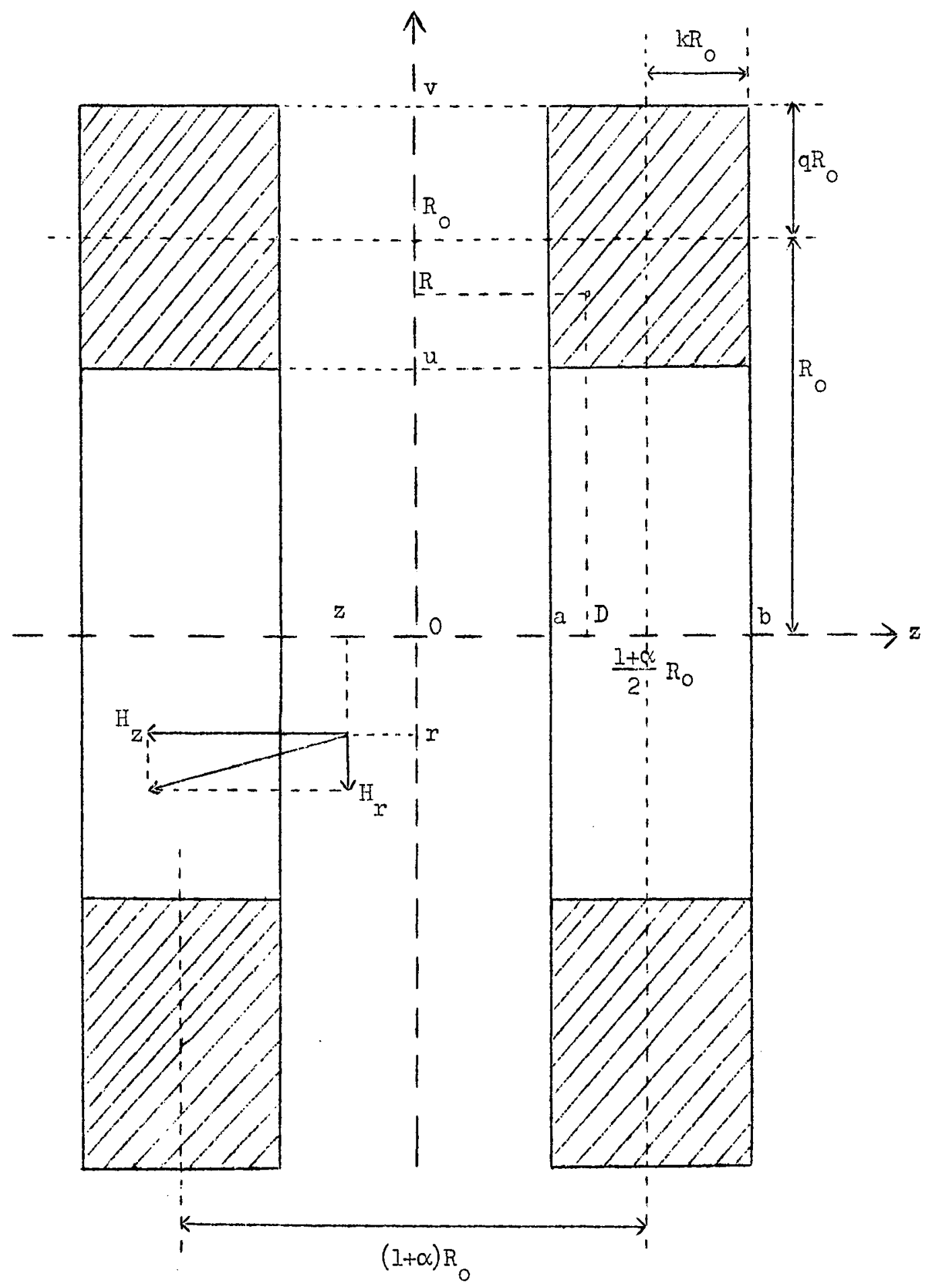

Bobines de Helmholtz 


\section{BIBLIOGRAPHIE}

(1) A. KASTJER - J. de Phys. (1950), 11, 255.

(2) J. BROSSEL et h. KiSTIER - C.R. (1949), 229, 1213.

(3) J. BROSSEL, P. SAGALYN et F. BITTER - Phys. Rev. (1950), 19, 196, 255.

I. BROSSEL et F. BITTER - Phys. Rev. (1952), 86, 311.

J. BROSSEL - (Thèse, Paris, 1951) - linn. de Phys. (1952), I, 622.

(4) J. BROSSEL, A. KASTJER et J.M. WINTER - J. de Phys. (1952), 13, 668.

J. BROSSEL, B. CAGNAC et A. KASTLER - C.R. (1953), 237, 984 .

J. BROSSEL, B. CAGNAC et A. KASTLER - J. de Phys. (1954), 15, 6.

(5) H,G. DEHMELT - Phys. Rev。(1957), 105. I487 et 1924.

W.E. BELL et A.L. BIOOM - Phys. Rev. (1957), 107, 1559。

W. FRANZEN et A.G. ENISLIE - Phys. Rev. (1957), 108, 1453.

W.E. BEILL et M.L. BLOOM - Phys. Rev. (1958), I09, 219.

M. LRDITI et T.S. CARVER - Phys. Rev. (1958), 109, 1012.

H.G. DEHMELT - Phys. Rev. (1958), I09, 381.

P. FRANKEN, R. SANDS et J. HOBART - Phys. Rev. Lett. (1958), 1, 52 。

R. NOVICK et H.E. PETERS - Phys. Rev. Lett. (1958), I, 54.

(6) A. KASTIER - Physica (1951), 17, 191.

i: $1:$ Proc. Phys. Soc. (1954), Al7, 353.

"11 J.O.S.li. (1957), 47, 460.

" 1 Suppl. Nuovo Cimento (1957), 6, 1148.

(7) J. M/ARGERIE, J. BROSSEL et A. K/STLER - C.R. (1955), 241, 474.

(8) QUANTUM ELECTRONICS - (édité par Ch.H. TOJNES, Columbia University Press, New York, 1960), J. BROSSEL p. 82.

(9) F. BITTER et J. BROSSEL - Phys. Rev. (1952), 85, 1051.

(10) F. BITTER, R,F. LACEY et B. RICHTER - Rev。 of Mod. Phys. (1953), 25, 174.

(11) J.P. BARRAT, J BROSSEL et A, KASTLER - C.R. (1954), 239, 1196.

A. BLANDIN et J.P.BARRAT - C.R. (1956), 243, 2041,

H.E. BELL et H.L. BLOON - Bull. Am. Phys. SOc. (1957), II,2, 384.

(12) B. CAGNAC, J. BROSSEI et A. KASTLER - C.R. (1958), 246, 1827.

B. CAGNAC - J. de Phys. (1958), 19, 863.

(13) B. CAGNAC et J. BROSSEL - C.R. (1959), ¿499, 77.

(14) A.C.G. MITCHELI et M.W. ZEMIAISKY - Resonance rodiation and excited atoms

(Cambridge University Press, Londres 1934), p. 95.

(25) M.A. GUIOCHON, J.E. BLAMONT et J. BROSSEL - C.R. (1956), 243, 1859.

$$
\text { " } 1 \text { is - J. de Phys. (1957), 18, } 99 .
$$
J.P. BARRAT - J. de Phys. (1959), 20, 657. 
(16) A.C.G. MITCHELI et M.W. ZFWANSKY - Resonance radiation and excited atoms (Cambridge University Press, Londres 1934), p. 146 et 147.

(17) H. SCHULER et J.E. KEYSTON - Zeit. f. Physik (1931), 72, 423.

H. SCHULER et Th. SCHMIDT - Zeit. f. Phys. (1935), 98, 239.

J。 BILISE et H. CHANTREL - J。 de Phys. (1957), I8, 193.

P.I. SAGALYN, A.C. MELISSINOS et F'. BITMER - Phys. Rev. (1958), 109, 375.

(18) A.C. MELISSINOS - Phys. Rev, (1959), 115, 126.

A.C. MELISSINOS et S.P. DAVIS - Phys. Rev. (1959), 115, 130.

(19) E.L. HILL - Proc. Nat. Hc. Sc. USA (1929), 15, 779.

D.R. INGLIS - Zeit. f. Phys. (1933), 84, 466.

(20) C. CASSIGNOL - J. de Phys. (1956), 17, supplément au $n^{\circ} 11$.

C. CASSIGNOL, G. RANC et R. SALVAUDON - Jo de Phys。 (1958), 19, 150A.

R. BERNLS et C. CASSIGNOL -. J. de Phys. (1960), 2], 566.

(21) W.F. MEGGERS - J. Opt. Soc. Am. (1948), 38, 7.

E. JACOBSEN et G.R. HARRISON - J. Opt. SOc. Im. (1949), 39, 1054.

W.F. MEGGERS et F.O. WESTFALI - J. of Research N.B.S. (1950), 44, 447 .

(22) 0. BUHL - Zeit. f. Phys. (1938), 109, 180 et 110, 395.

F. BITTER, H. PLOTKIN, B. RICHTER, A. TEVIOTDLIE et J.E.R. YOUNG -

Phys. Rev. (1953), 9.1, 421.

F. BITTER, S.P. DAVIS, B. RICHTER et J.E.R. YOUNG - Phys.Rev。(1954), 96,

(23) Nelly ROLLET - Diplôme d'Études Supérieures, Paris 1955.

(24) C. COHEN-TANNOUdJI - Diplôme d'Études Supérieures, Paris 1955

(25) W.G. PROCTOR et F,C. YU - Phys. Rev. (1951), 81, 20.

(26) P.GRIVET, M, SOUTIF et M。BUYLE-BODIN - C.R. (1949), 229, 113.

M. SOUTIF - (Thèse, Paris 1950) - Revue Scientifique (1951), 89, 203.

(27) P. GRIVET et alii. - Lc. résonance paramagnétique nucléaire (C.N.R.S., Paris 1955), p. 143.

(28) W. FAUST, M. MacDERWOTT et W. LICHTEN - Bull. fin. Phys. Soc. (1958), II,3,

M.N. MacDERMO'I' - 'l'hèse, columbia University, 1959.

p. 371 .

(29) R.H. KOHLER - Thèse,Massachusets Institute of Technology, 1960.

C.V. STAGER - Thèse, Massachusets Institute of Technology, 1960.

(30) V.E. LIMB - Phys. Rev。(1941), 60, 817.

(31) H.A. THOMAS, R.L. DRISCOLL et J.A. HIPPID - Phys. Rov. (1950), 78, 787.

W. WILHELNY - Ann. der Phys. (1957), I9, 329.

R.L. DRISCOLL et P.L. BENDER - Phys. Rev. Lett. (I958), 11, 413. 
(32) E.R. COHEN, J.W.M. DU MOND, T.W. LAYTON et J.S. ROLLET - Rev. Mod. Phys. (1955), 27, 363.

E.R. COHEN, J.W.M. DU MOND - Phys. Rev. Lett. (1958), 1, 291 et 382.

(33) H.SOMMER, H.A. THONAS et J.A. HIPPLE - Phys. Rev. (1951), 82, 697. D.J. COLLINGTON, k.N. DELLIS, J.H. SANDERS et K.C. TURBERFIELD - Phys. Rev. K.R. TRIGGER - Bull. im. Phys. Soc. (1956), II, I, 220. (1955) 99, 1622

(34) F. BLOCH - Phys. Rev. (1946), 70, 460.

(35) W. FRANZEN - Phys. Rev. (1959), 115, 850.

(36) H.C. TORREY - Phys. Rev. (1949), 76, 1059.

(37) I.I. RfiBI - Phys. Rev. (1937), 51, 652.

(38) J.M. WINTER - (Thèse Paris, 1958) - Ann. de Phys. (1959), 4, 745.

(39) E. MUJORANA - Nuovo Cimento (1932), 2, 43.

(40) F. BLOCH et A. SIEGERT - Phys. Rev. (1940), 57, 522.

(41) R.K. WINGSNESS et F. BLOCH - Phys. Rev. (1953), 89, 728. F. BLOCH - Phys. Rev. (1956), 102, 104. F. BLOCH - Phys. Rev. (1957), 105, 1206.

(42) J.P. BARRkiT et C. COHEN-TANNOUDJI - à paraître.

(43) N. BLOEMBERGEN, E.M. PURCELL et R.V. POUND - Phys. Rev. (1948), 73, 679. Y. LAYANT - J. de Phys. (1955), 16, 411. A. LBRAGM - Cours de résonance magnétique nucléaire.

(44) H.G. DEHELTT - Bull. Am. Phys. Soc. (1958), II,2, 9.

(45) J.F. WAYMOUTH, S.W. THOMPSON, L.C. BRADLEY et H.H. STROKE - Quarterly Progress Report of M.I.T. (1960), 58, 102.

(46) F. BLOCH - Phys. Rev. (1954), 24, 496.

(47) S. MILLMANn - Phys. Rev. (1939), 55, 628.

(48) H.G. DFHMELT, H.G. ROBINSON et W. GORDY - Phys. Rev. (1954), 93, 480. K. MURAKANA - Phys. Rev. (1955), 98, 1285. R.V. POUND et G.K. WERTHEIM - Phys. Rev. (1956), 102, 396.

(49) A.C.G. MITCHELL et W. ZEMIANSKY - Resonance Radiation and Excited itoms, p. 200

(50) C.H. TOWNES et B.P. DAILEY - $\dddot{3}$. Chem. Phys. (1952), 20, 35. 1.. HONIG, M. MiNDER, M.L. STITCH et C.H. TOWNES - Phys. Rev. (1954), 96, 627.

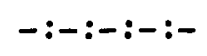


Fig. 1

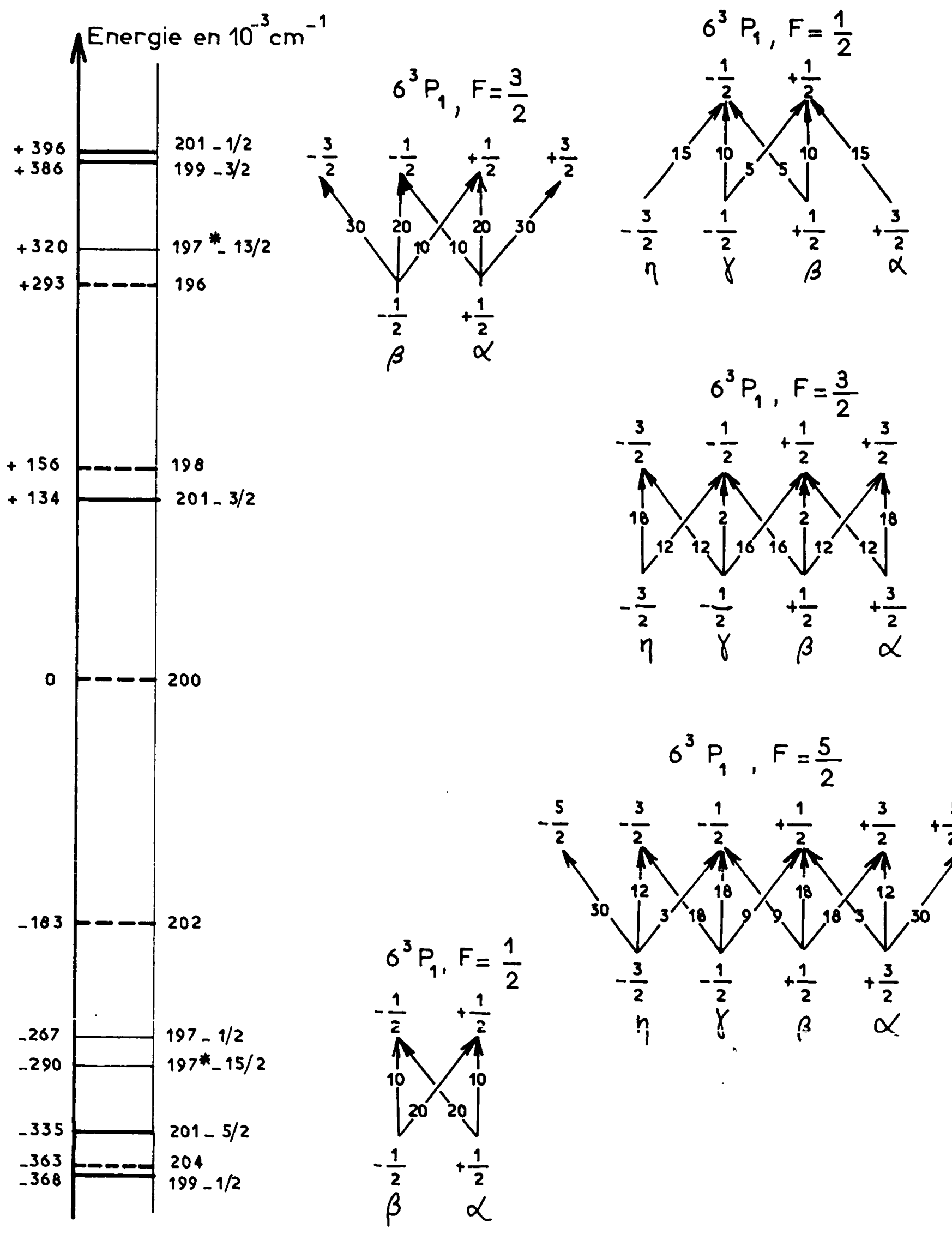

Structure fine de la raie $2537 \AA$
Composantes Zeeman de ${ }^{199} \mathrm{Hg}$
Composantes Zeeman de ${ }^{20} \mathrm{Hg}$ 


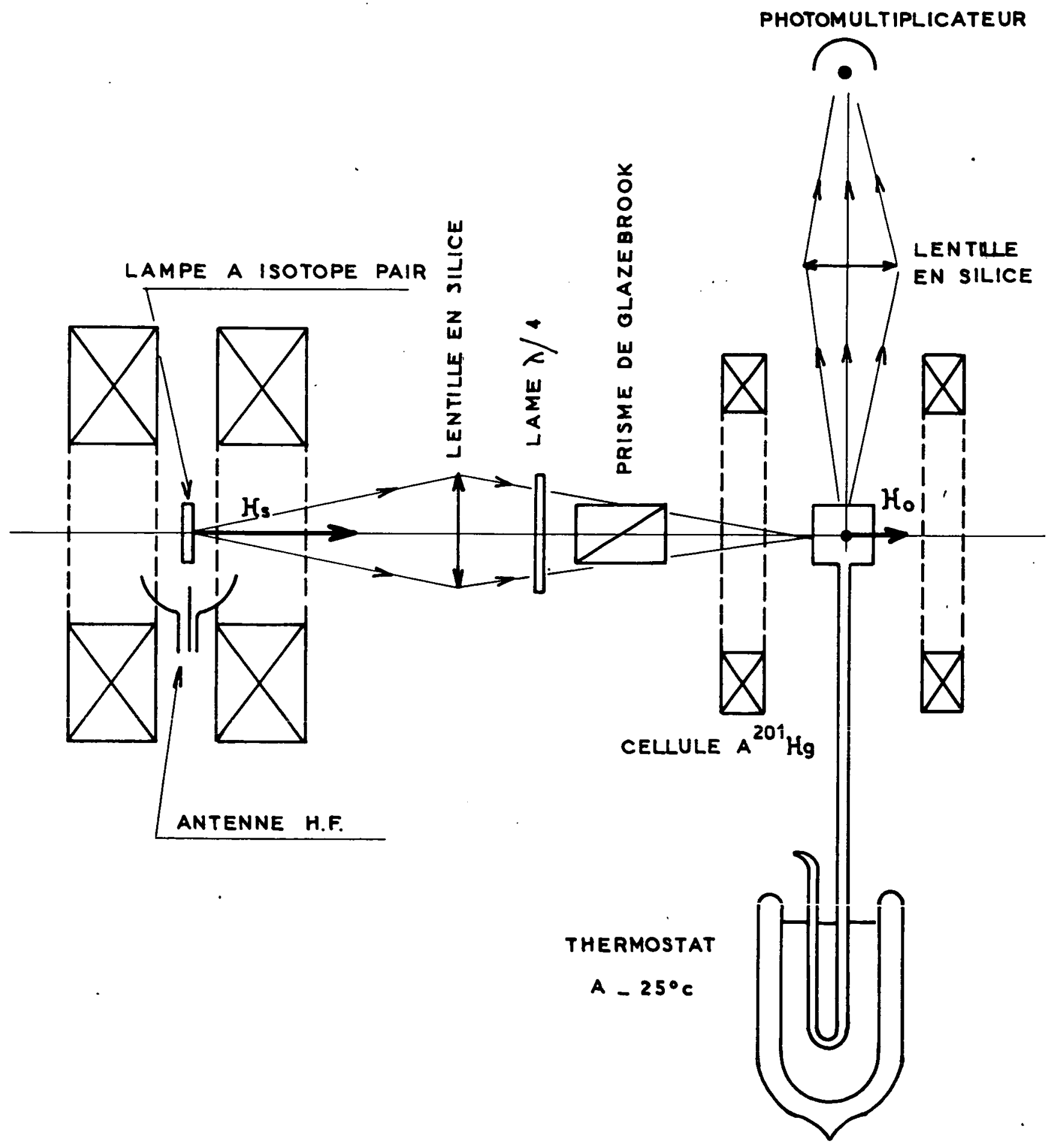


BALAYAGE MAgNetique AVEC

une CELluLE A ${ }^{201} \mathrm{Hg}$

Fig. 3

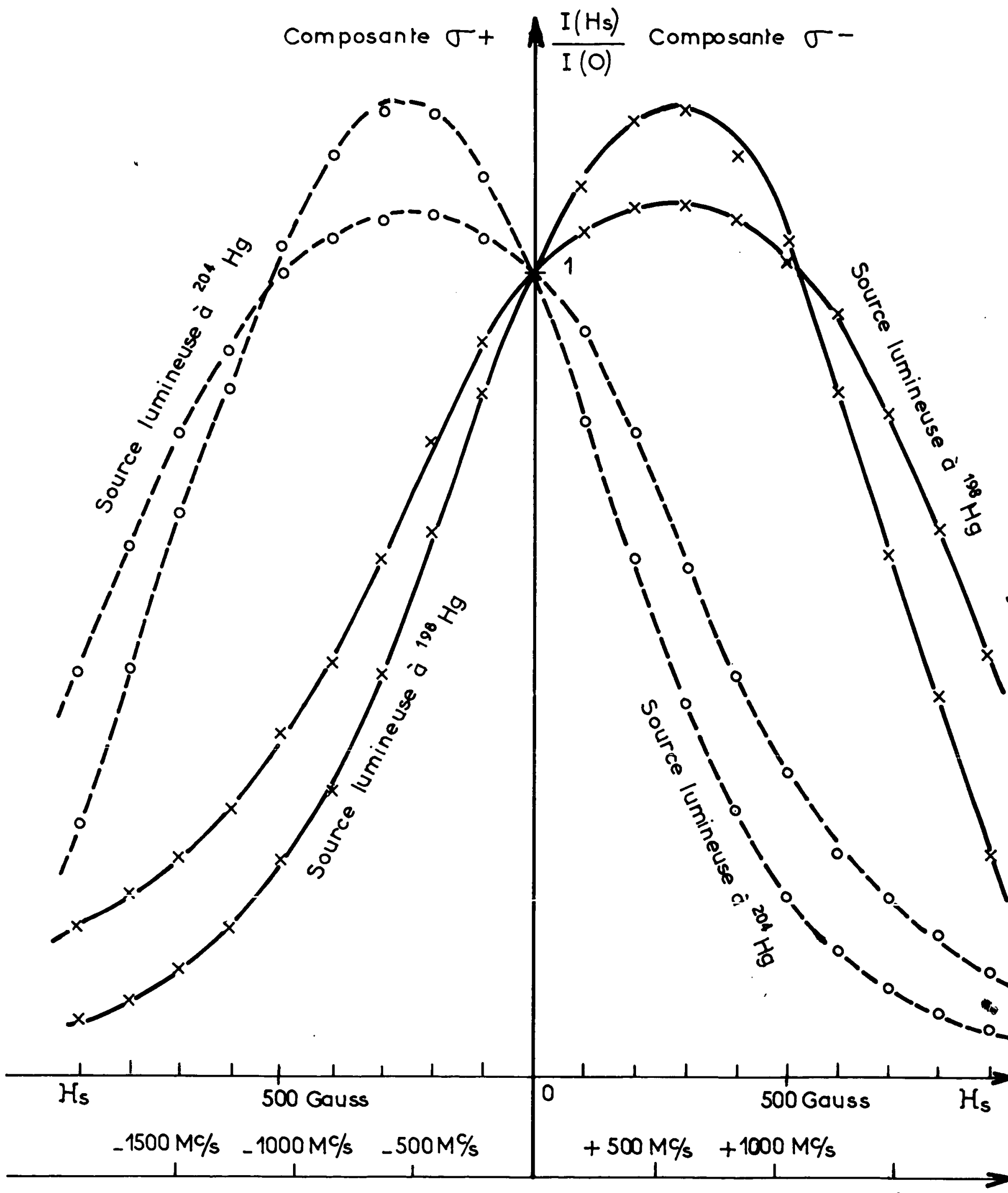

Fréquence 


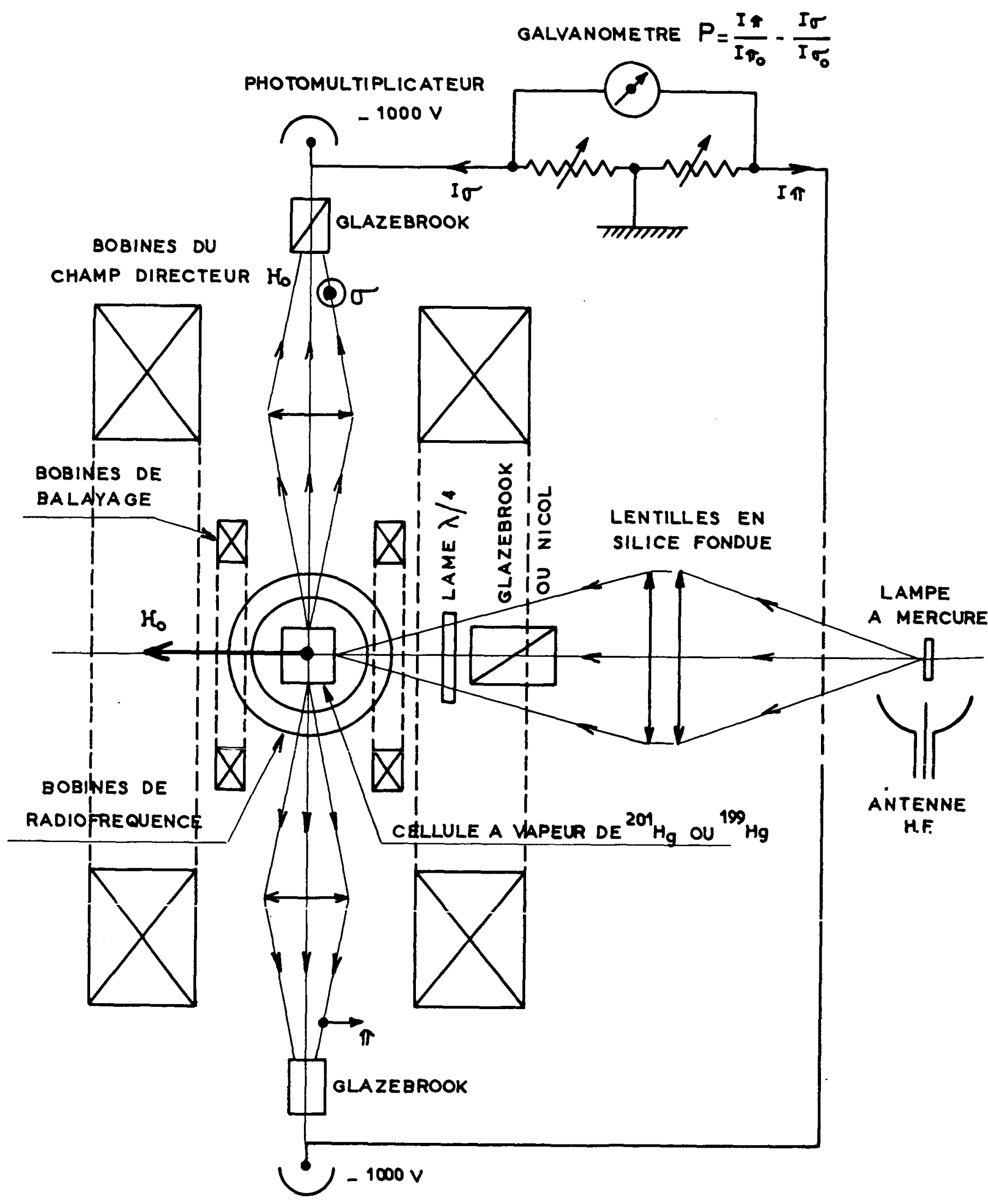




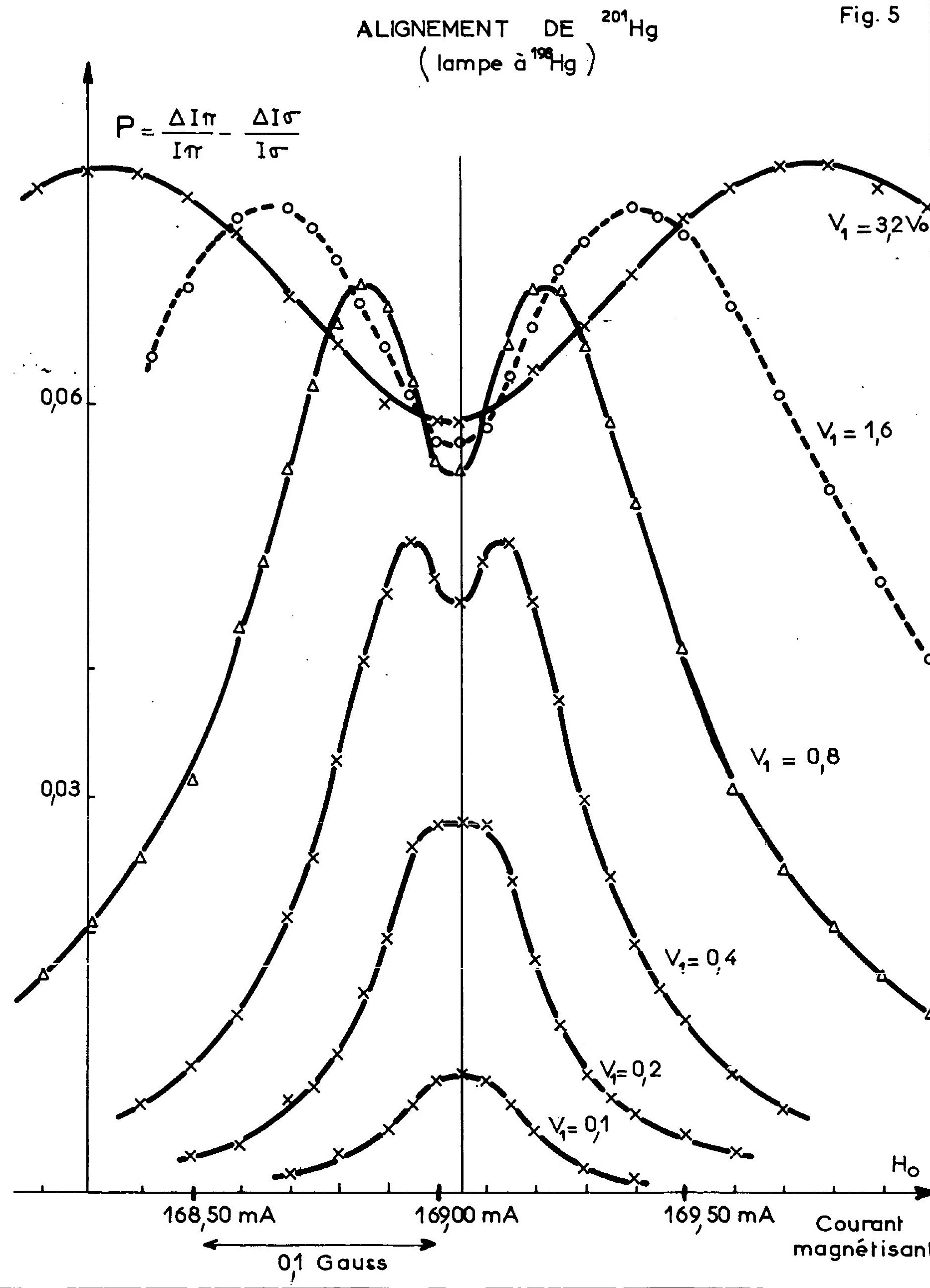


ORIENTATION DE ${ }^{201} \mathrm{Hg}$

Fig. 6

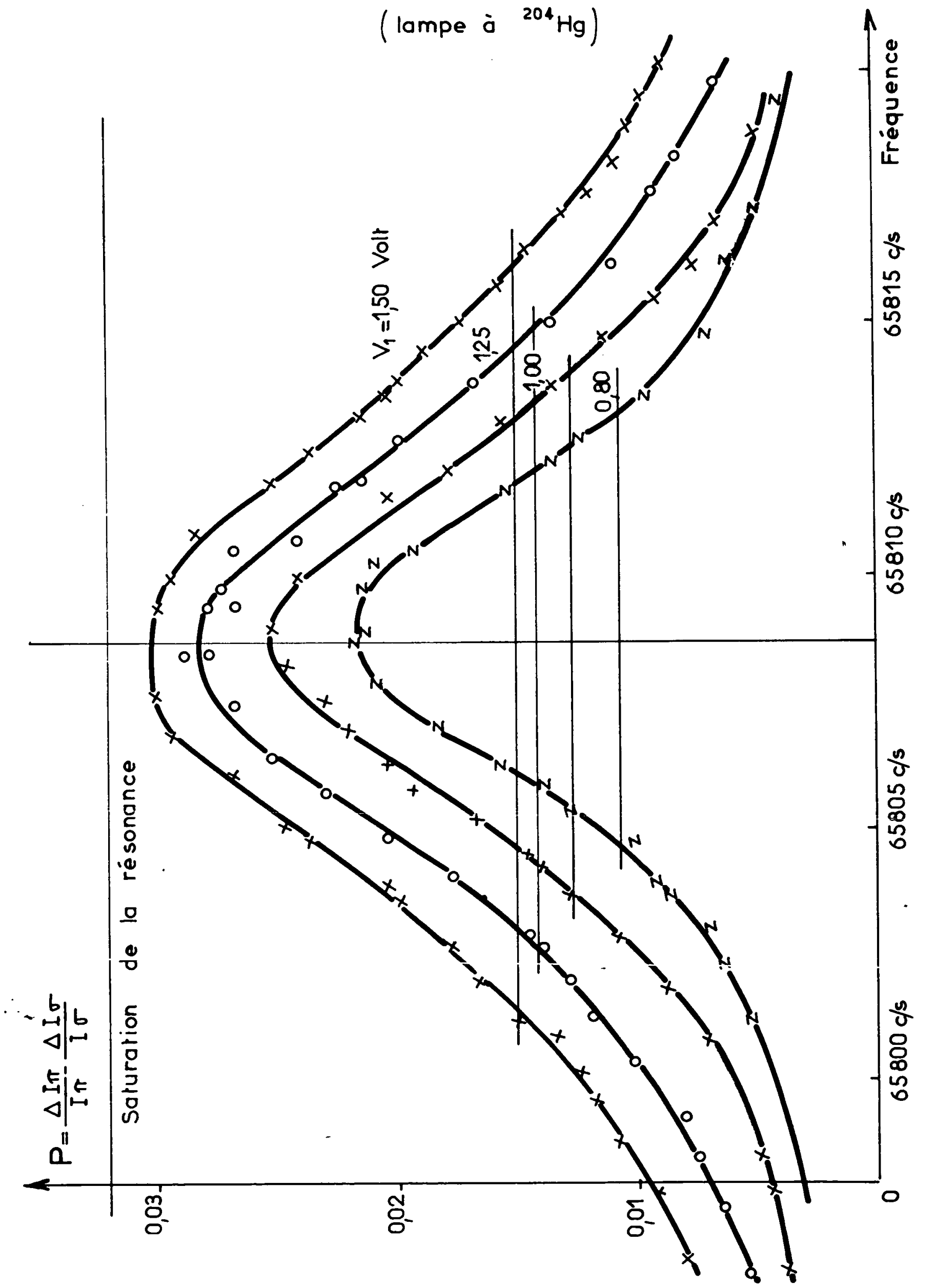




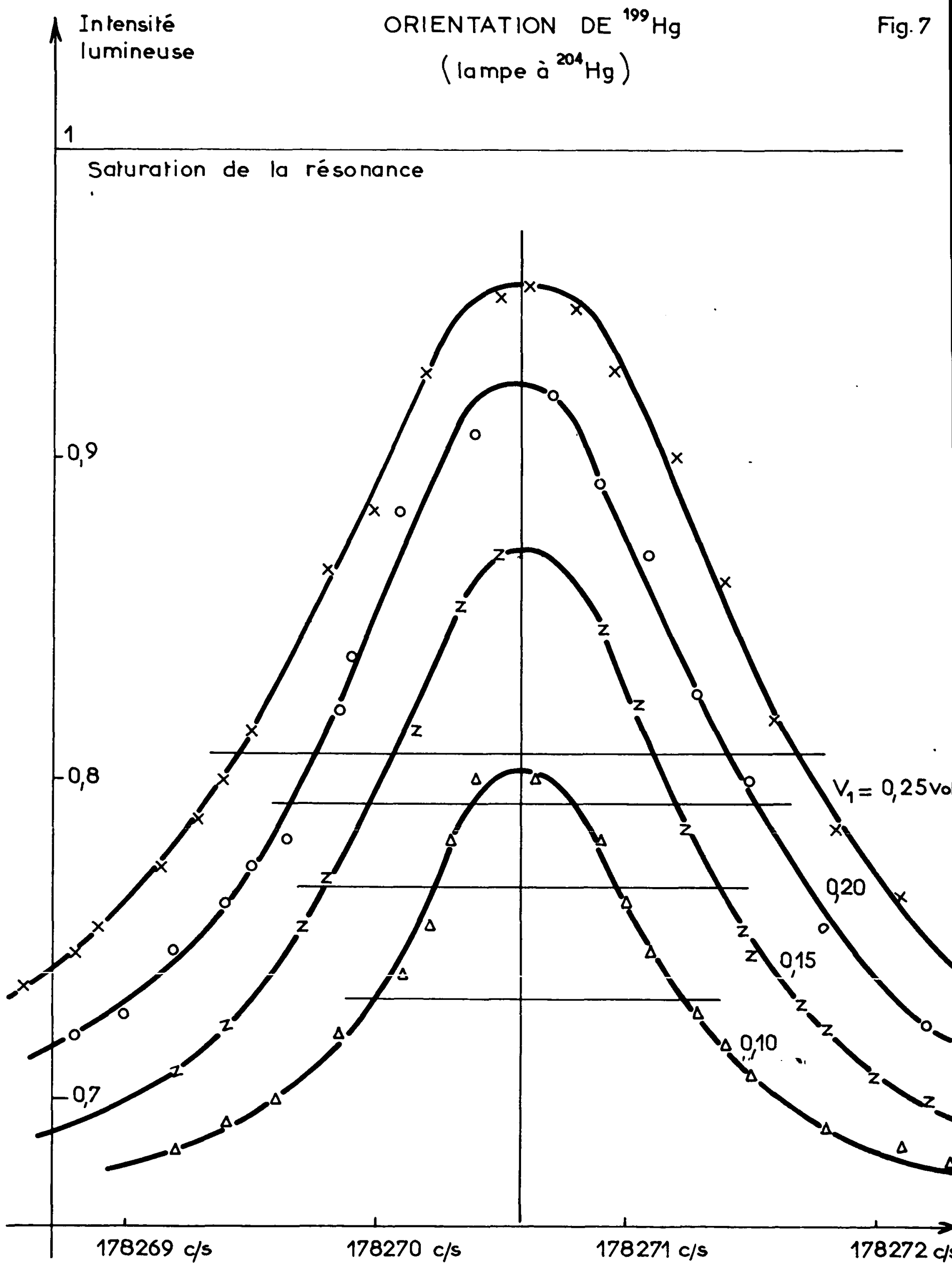




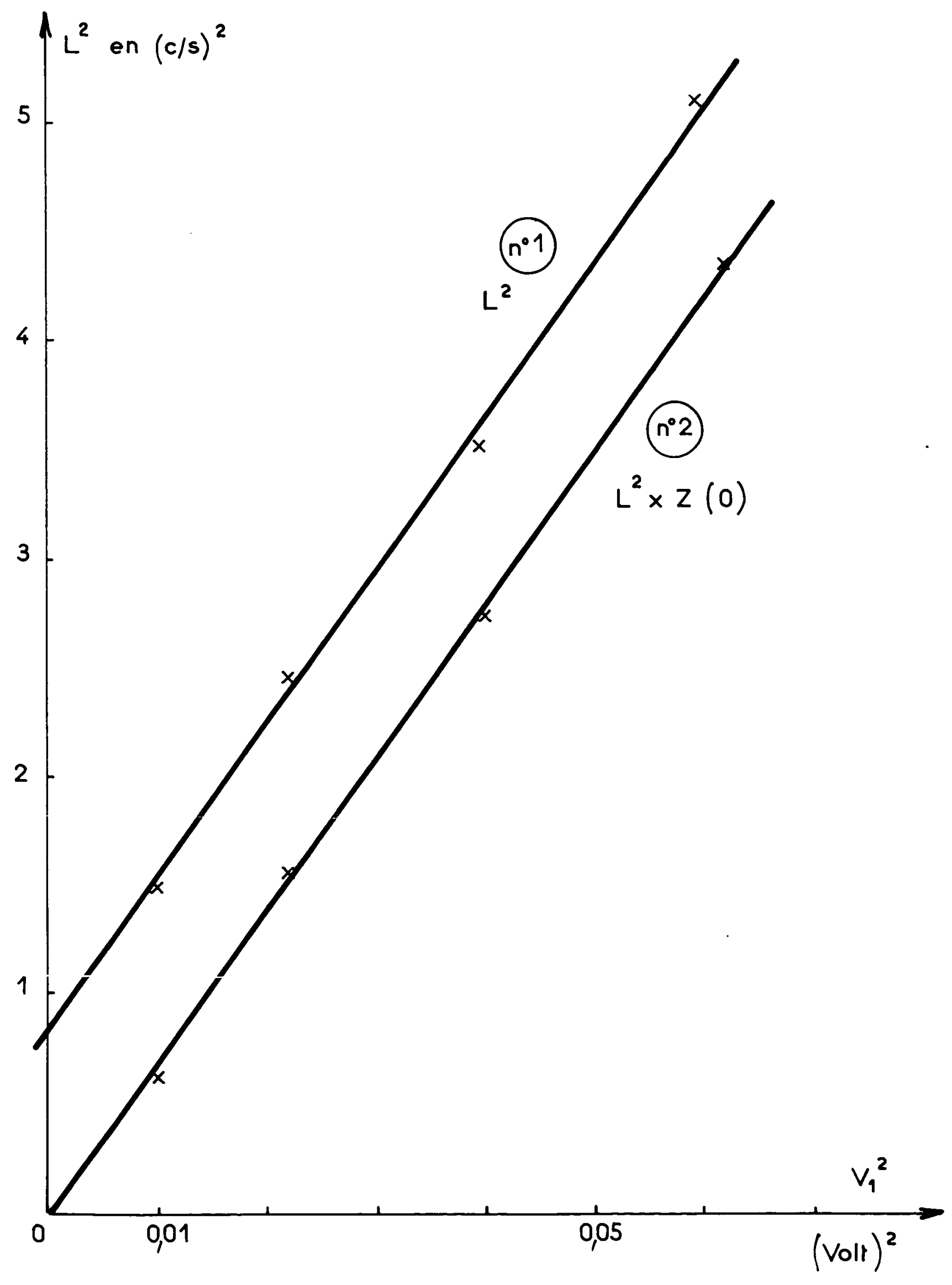




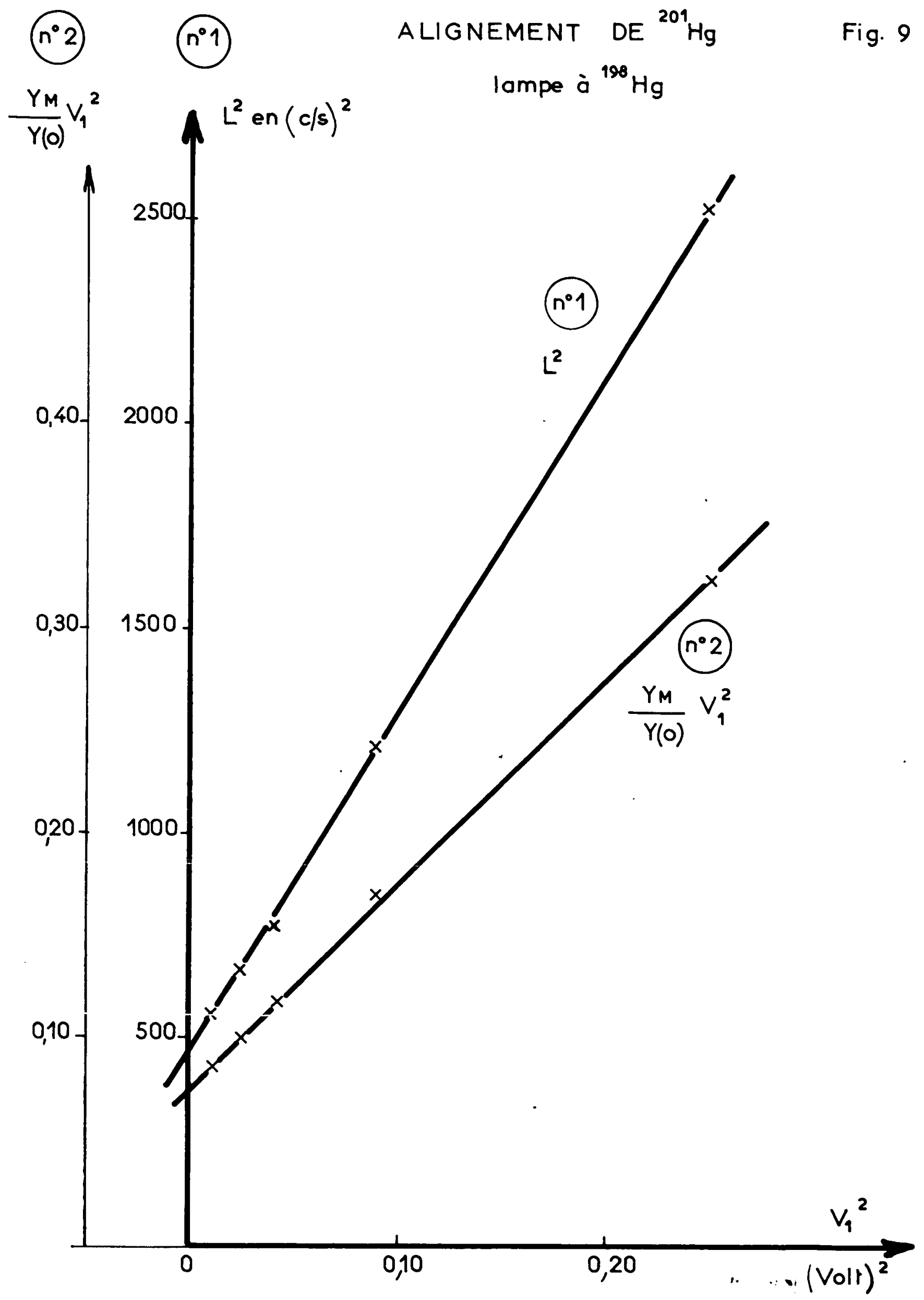


ALIGNEMENT DE ${ }^{201} \mathrm{Hg}$

Fig. 10

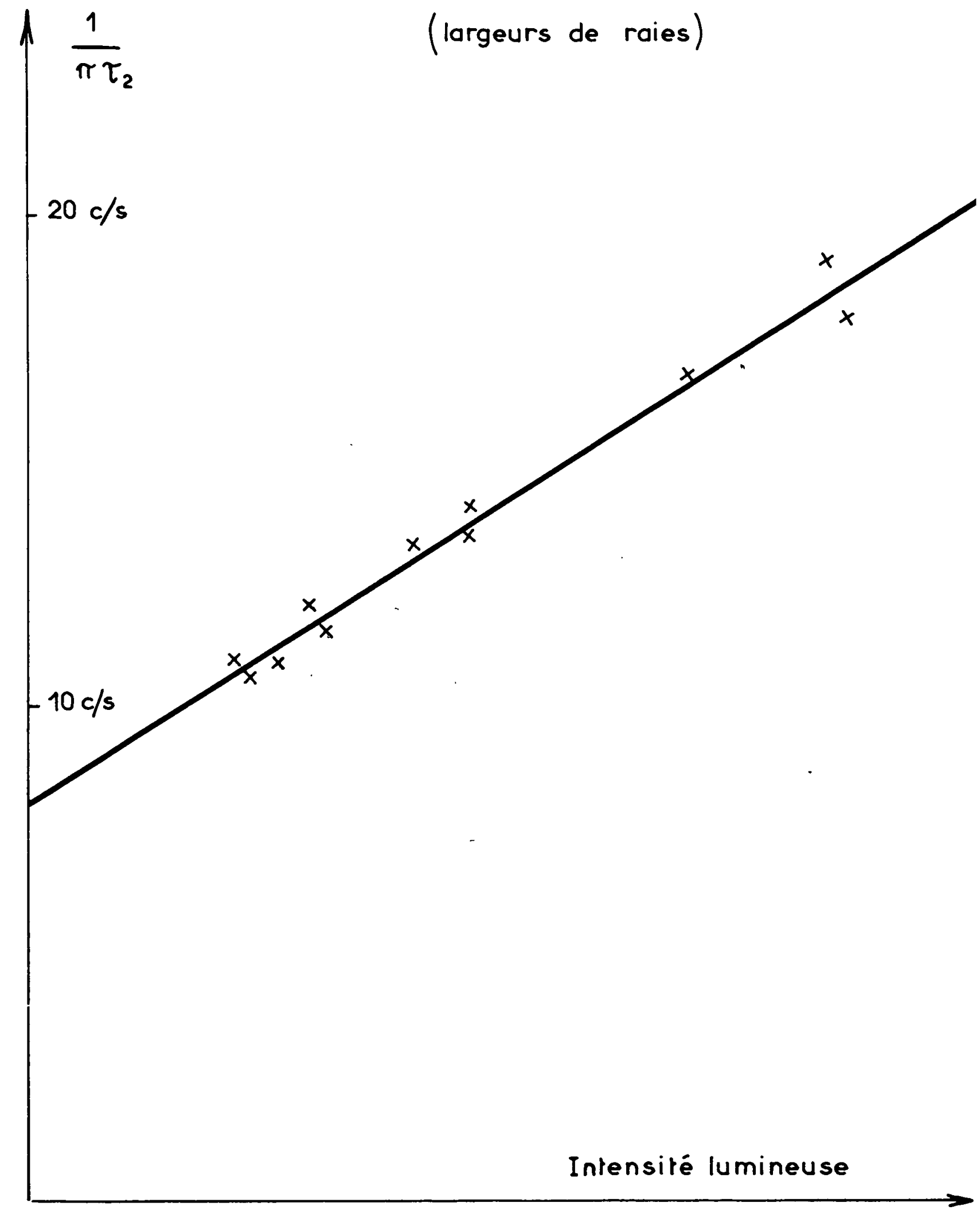




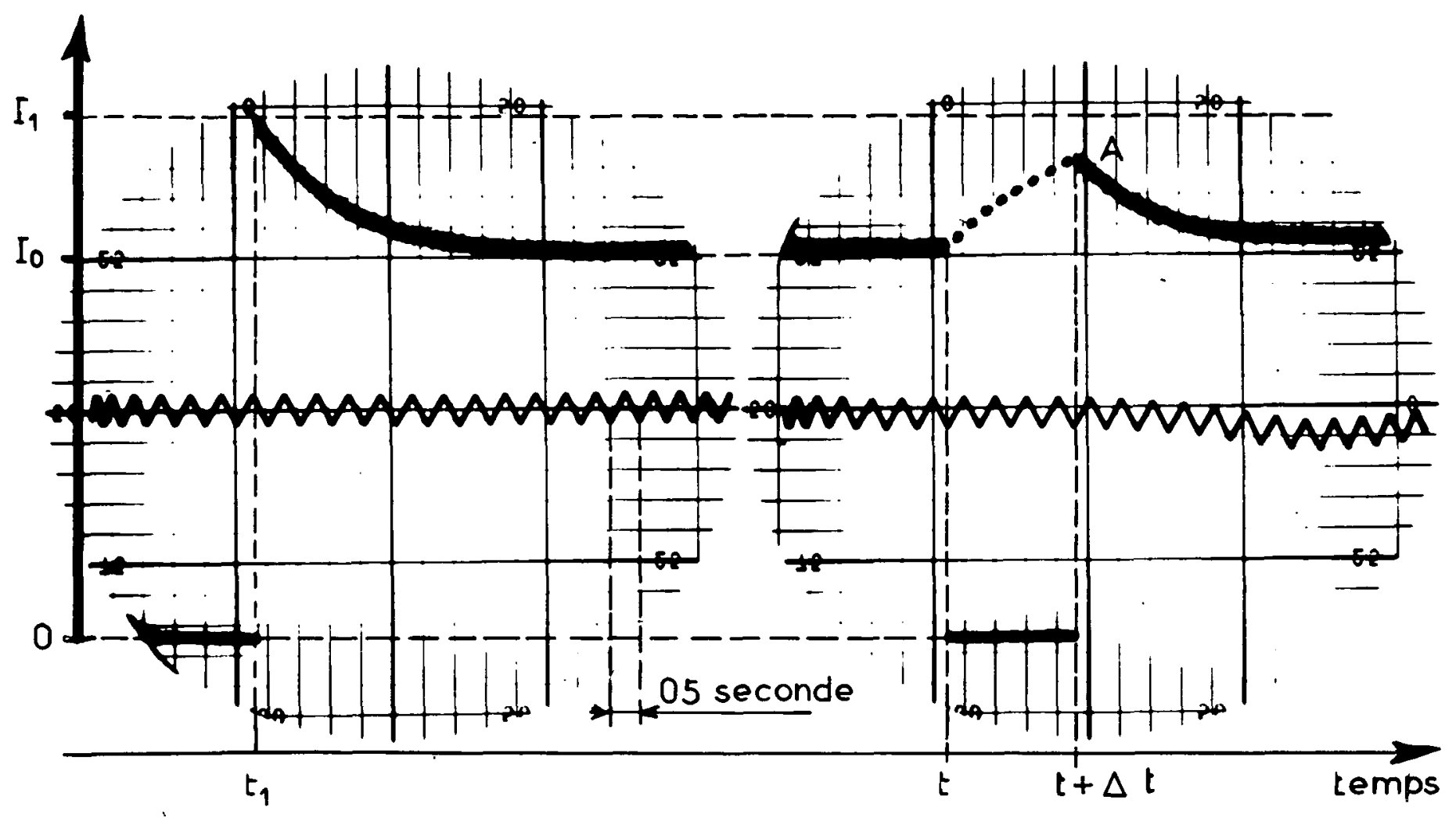

Fig. 110

Fig. $11 b$

Fig.11c

Fig. 11d
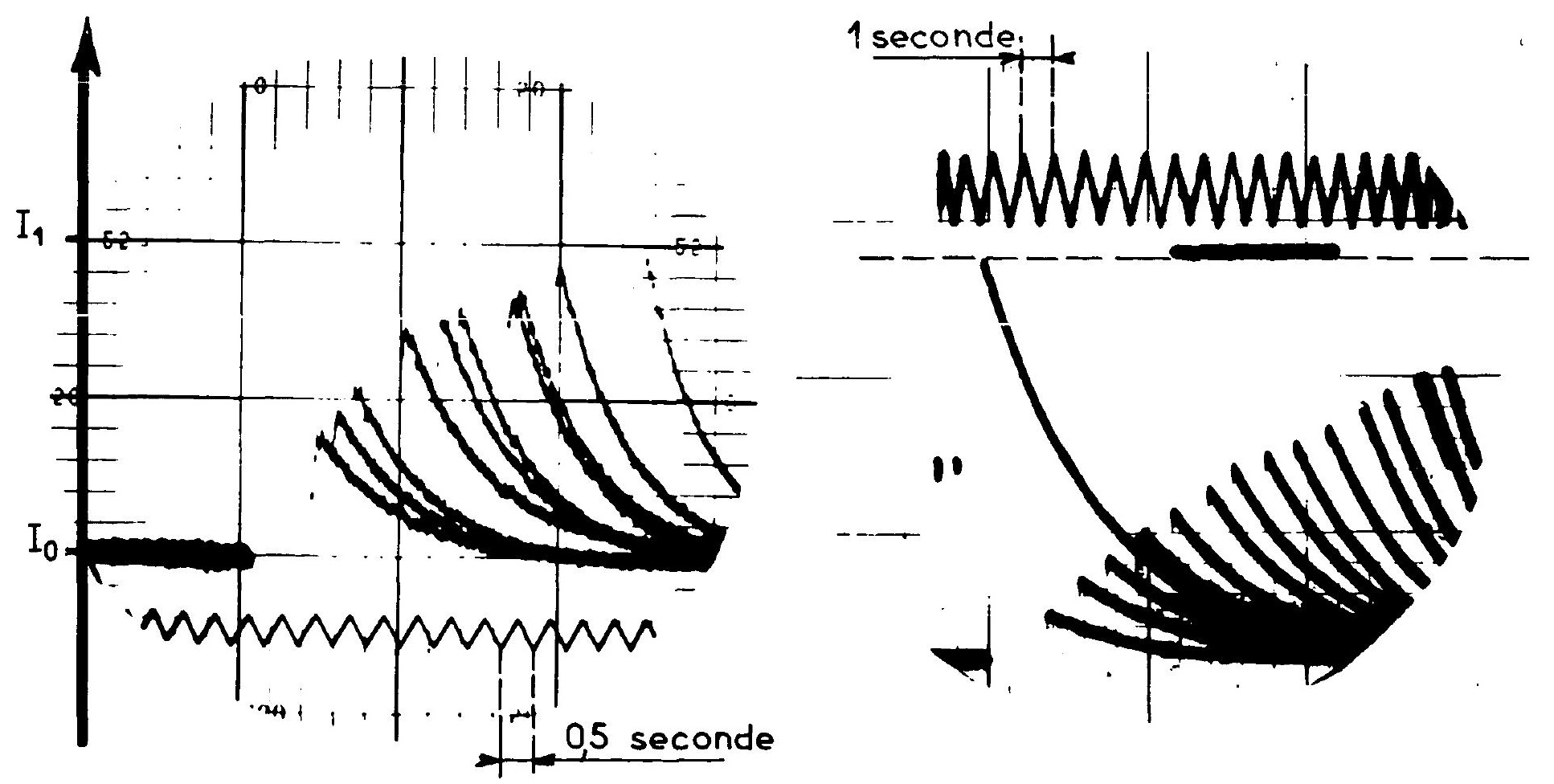


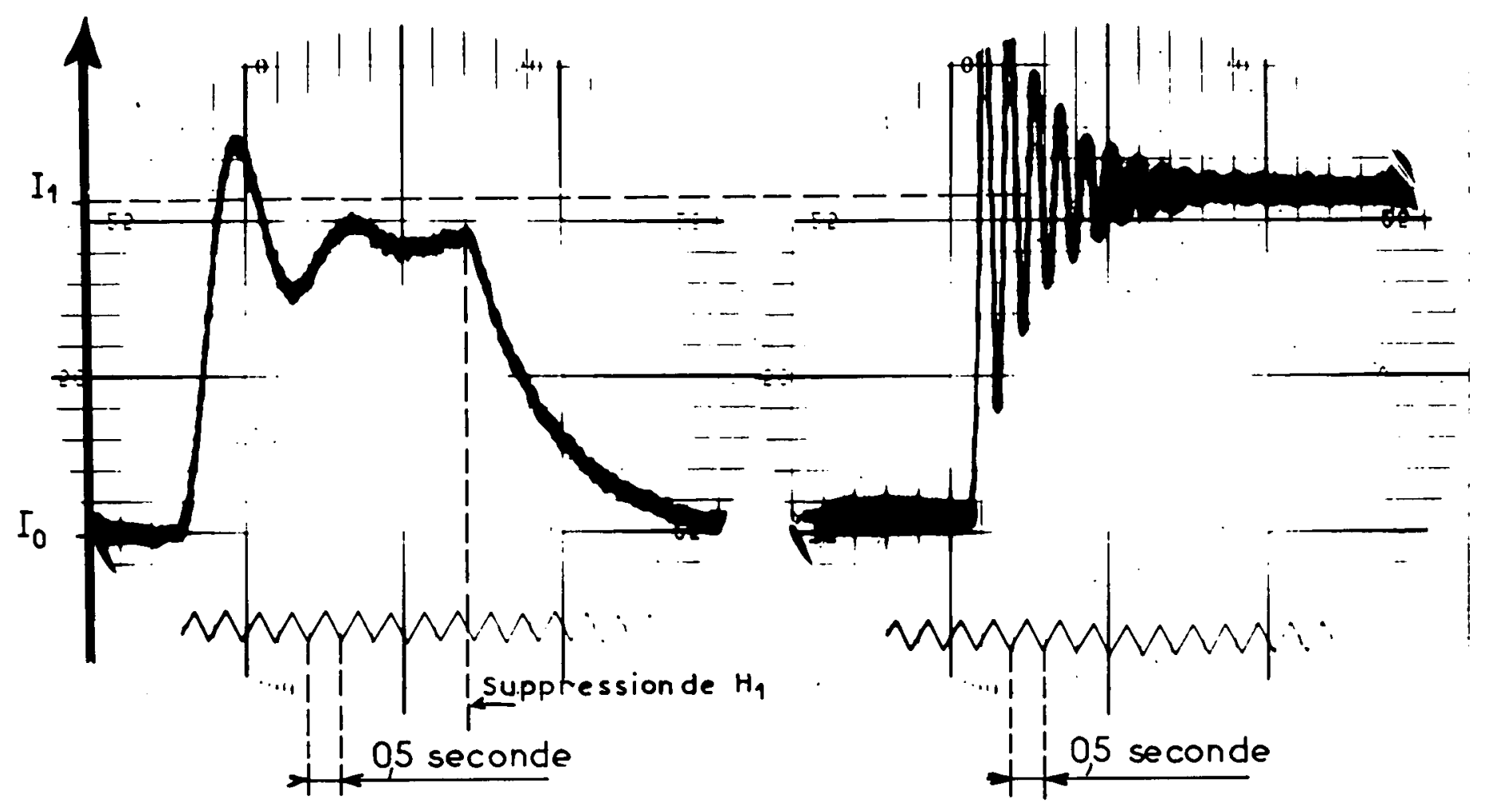

Fig. $12 a$

Fig. 12 b

Fig. $12 \mathrm{c}$

Fig. $12 \mathrm{~d}$

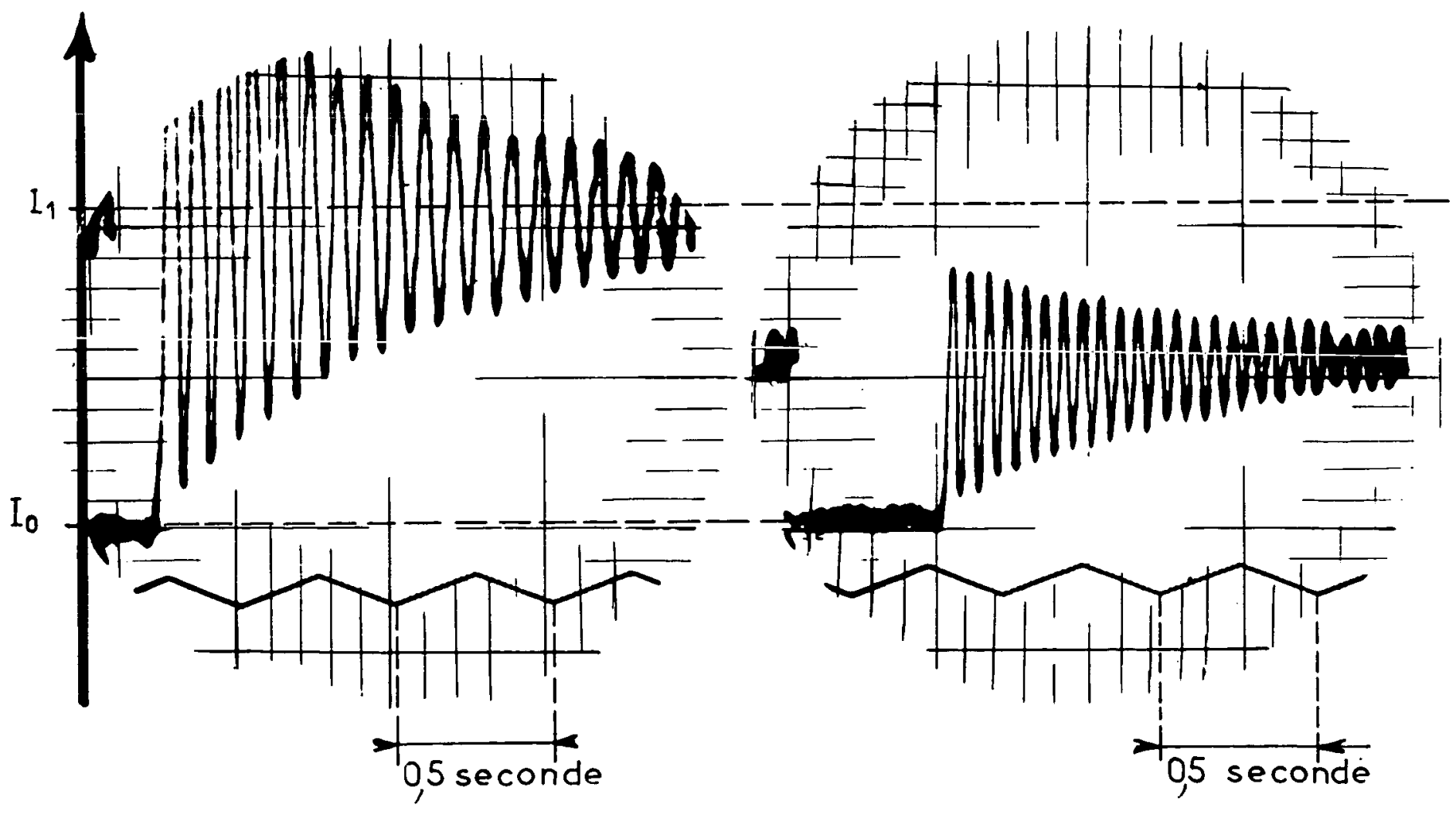




$$
\frac{2}{\Theta}=\frac{1}{T_{1}}+\frac{1}{T_{2}}
$$

Fig. 13 a

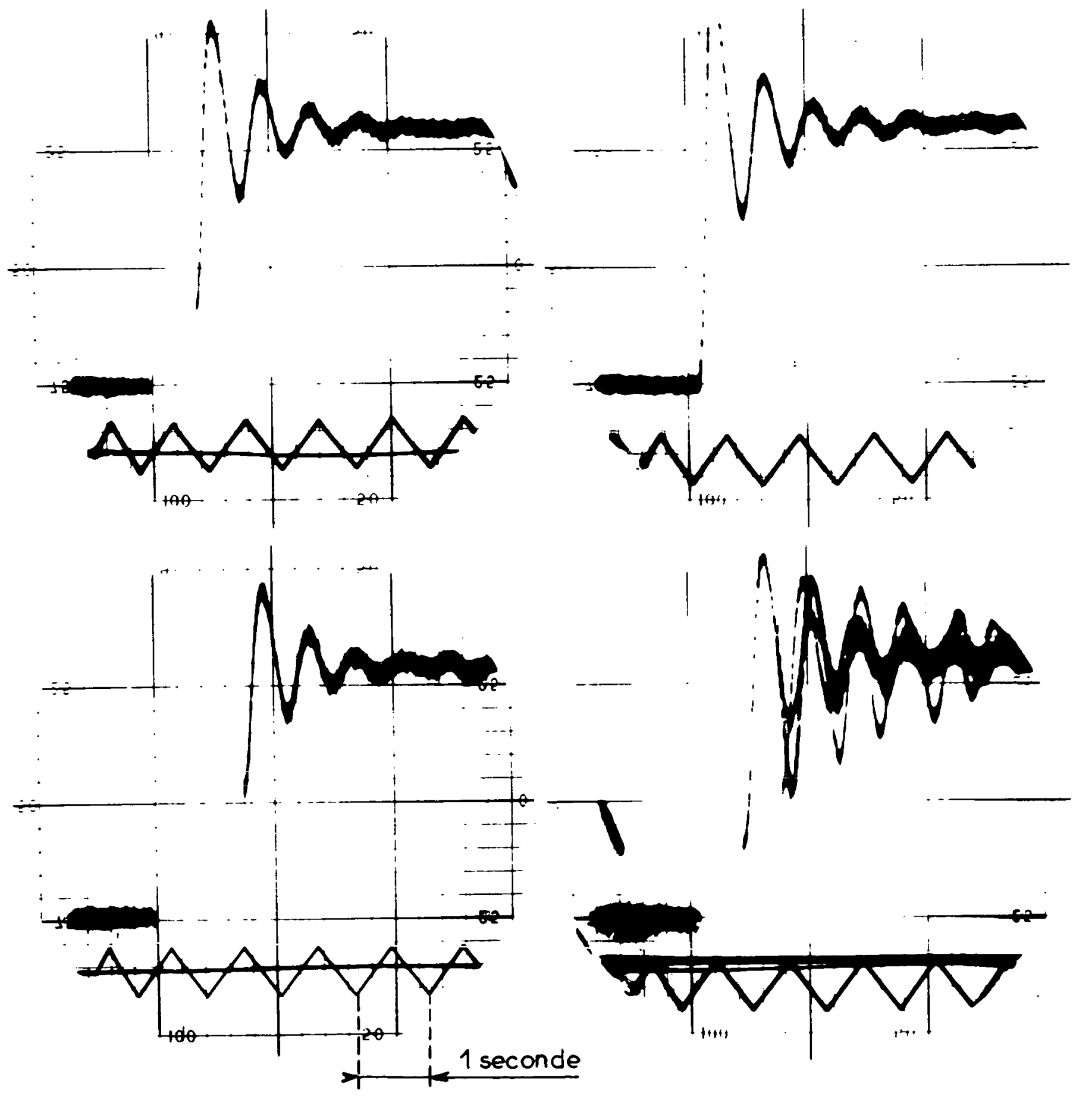

Fig. $13 c$
Fig. $13 b$ 
(lampe $a^{204} \mathrm{Hg}$ - Transitoires)

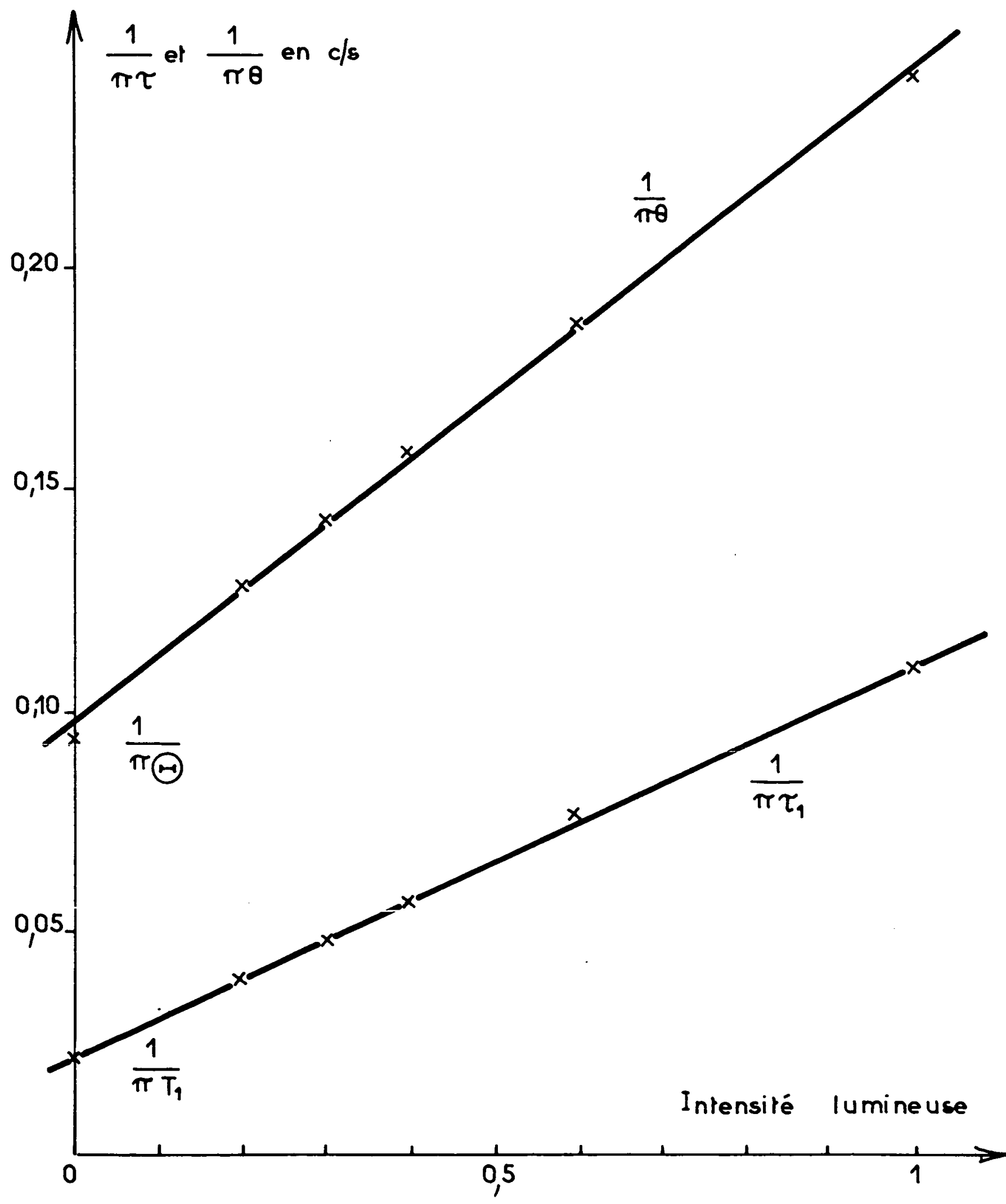


Fig. 15

Fig. 15 a
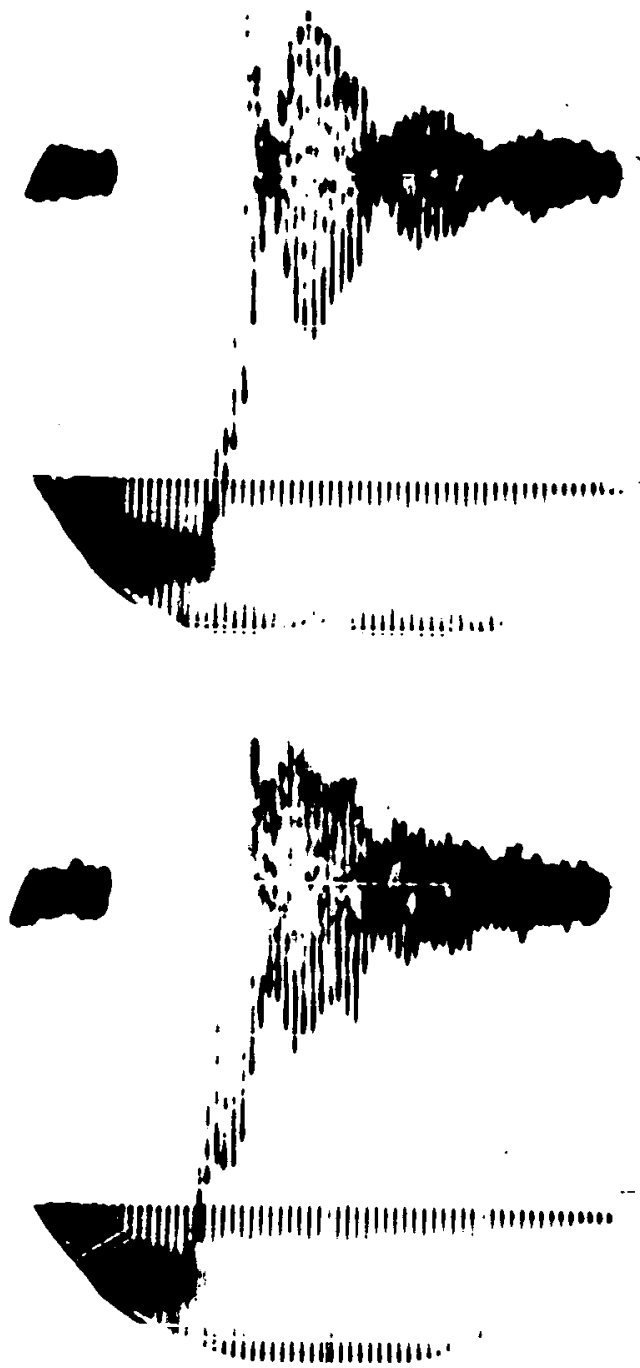

Fig $15 c$
Fig. 15 b

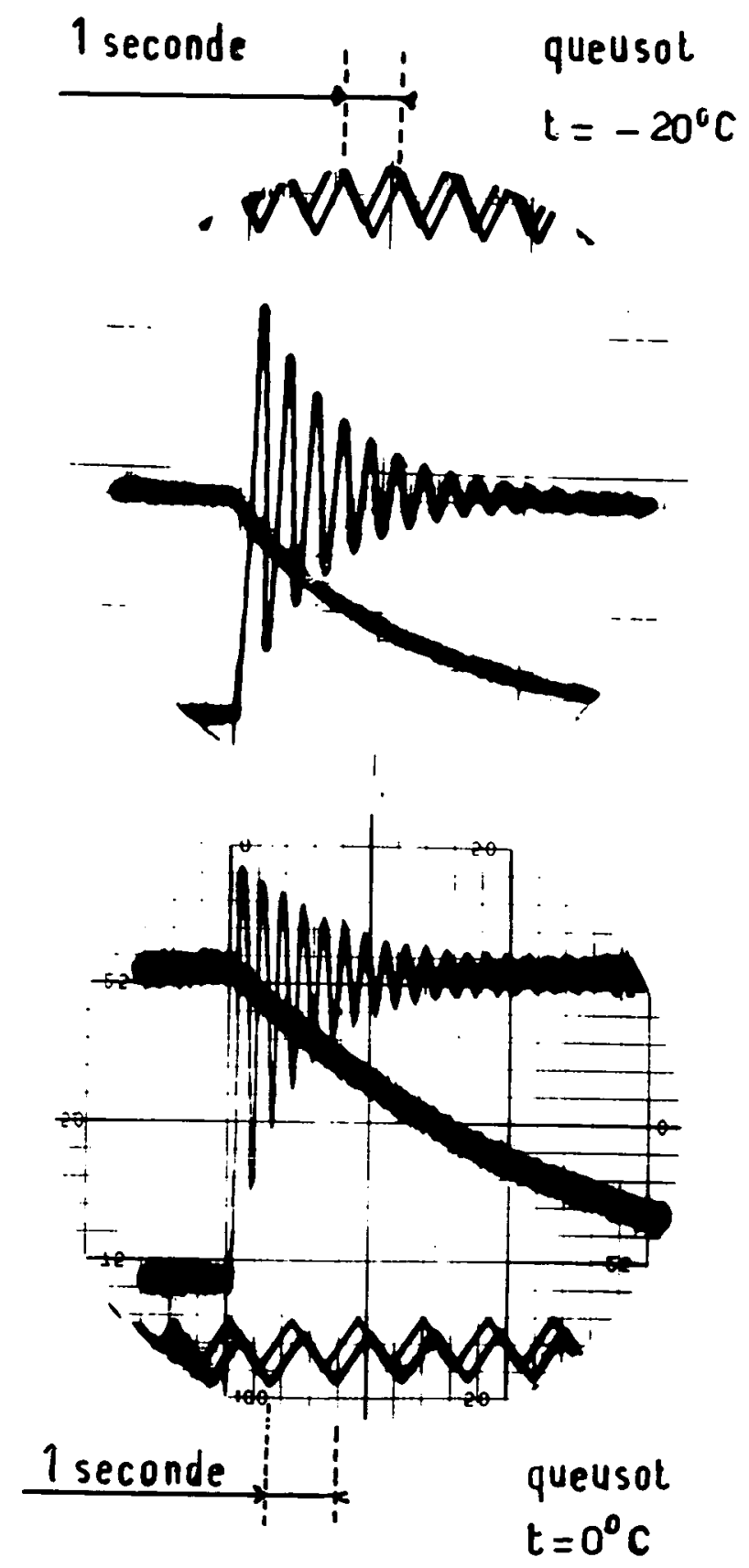

Fig $15 d$ 
Fig. 16

Fig. 16 a

Fig.16 b

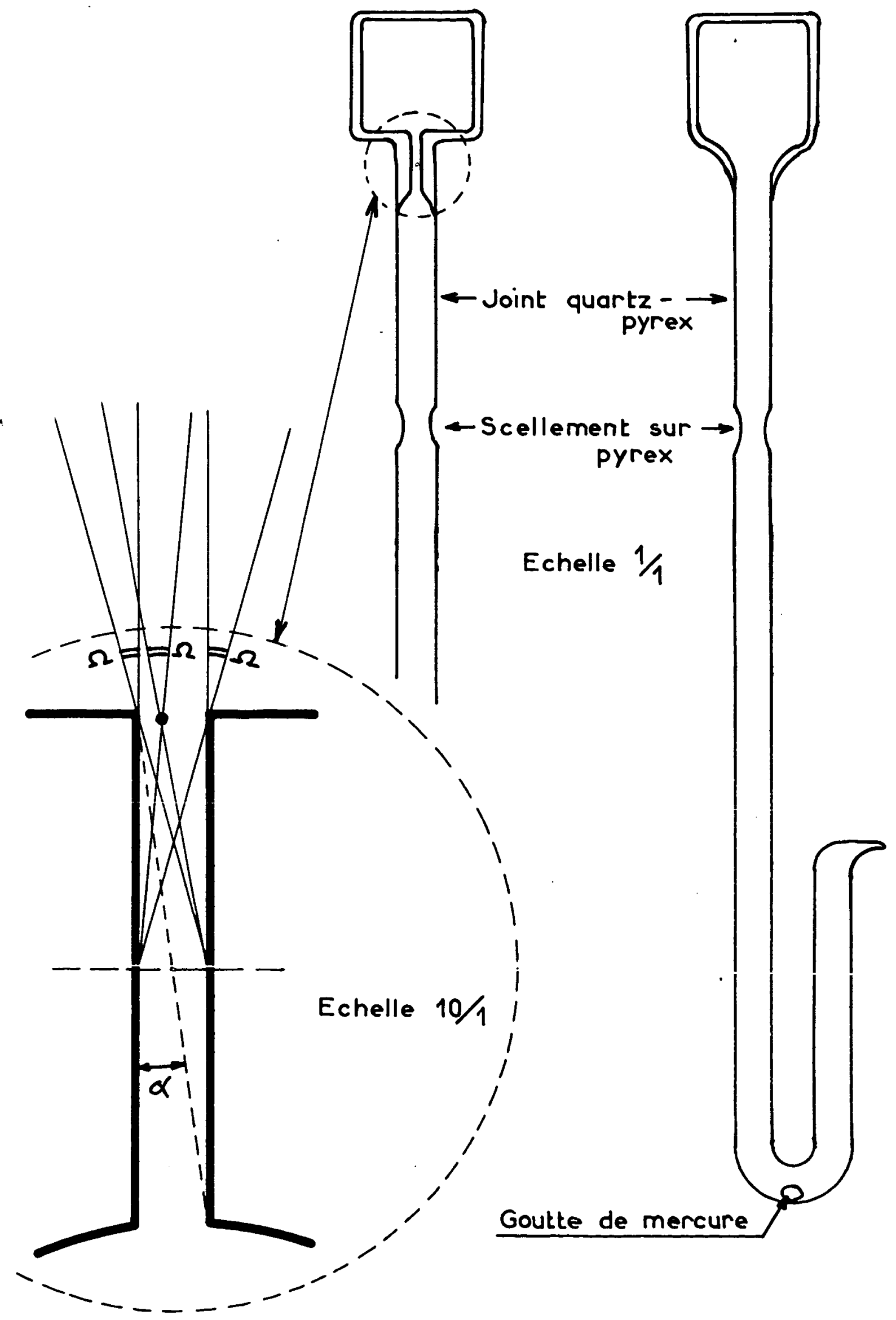




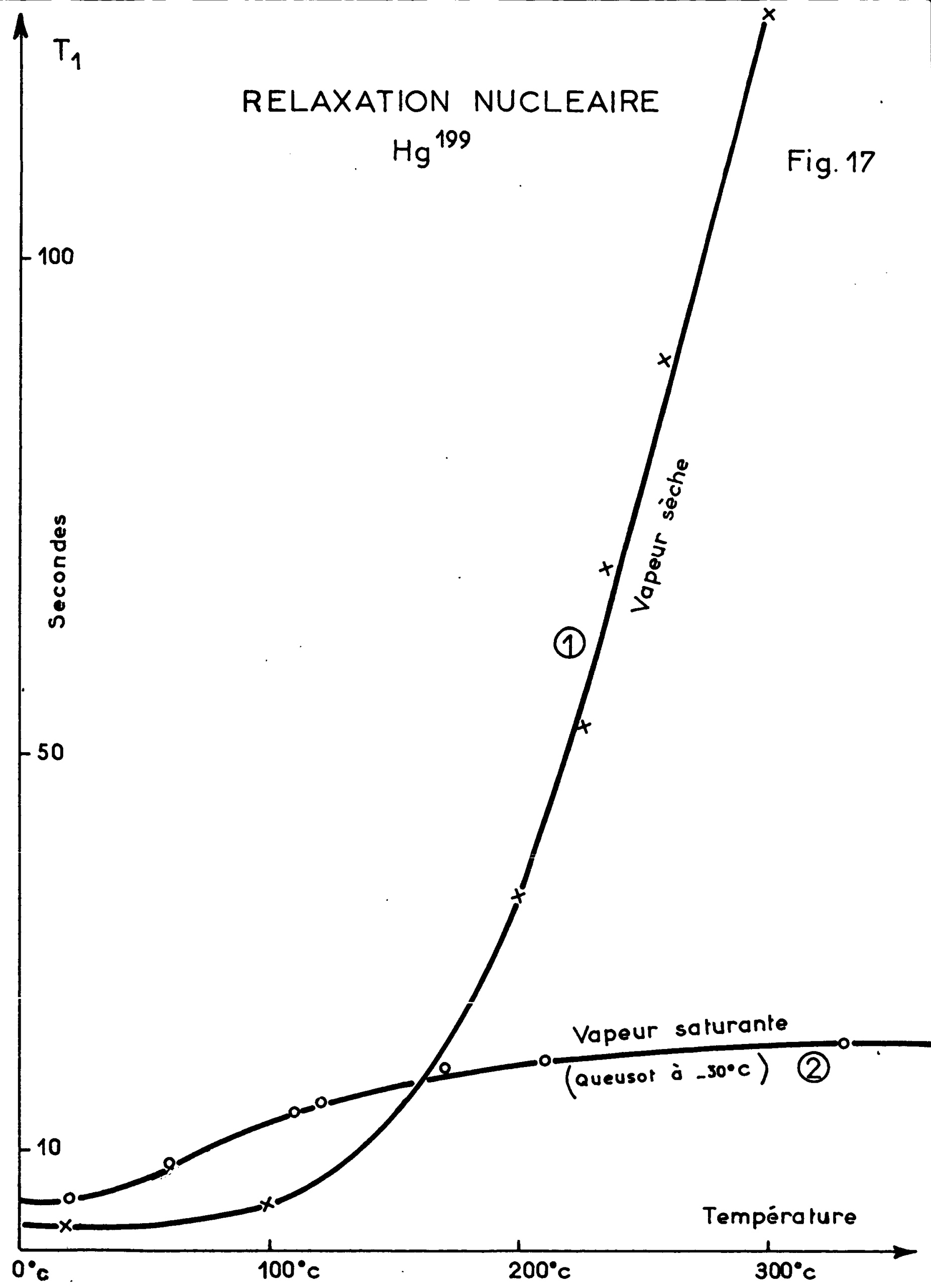




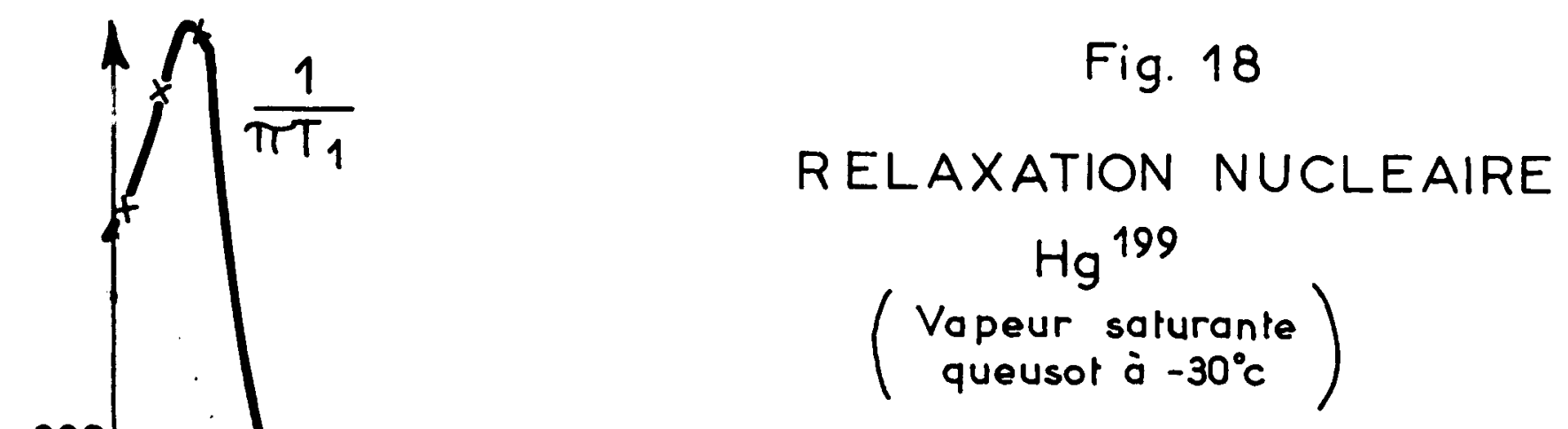

(3) Cellule à $20^{\circ} \mathrm{C}$ après chauffage
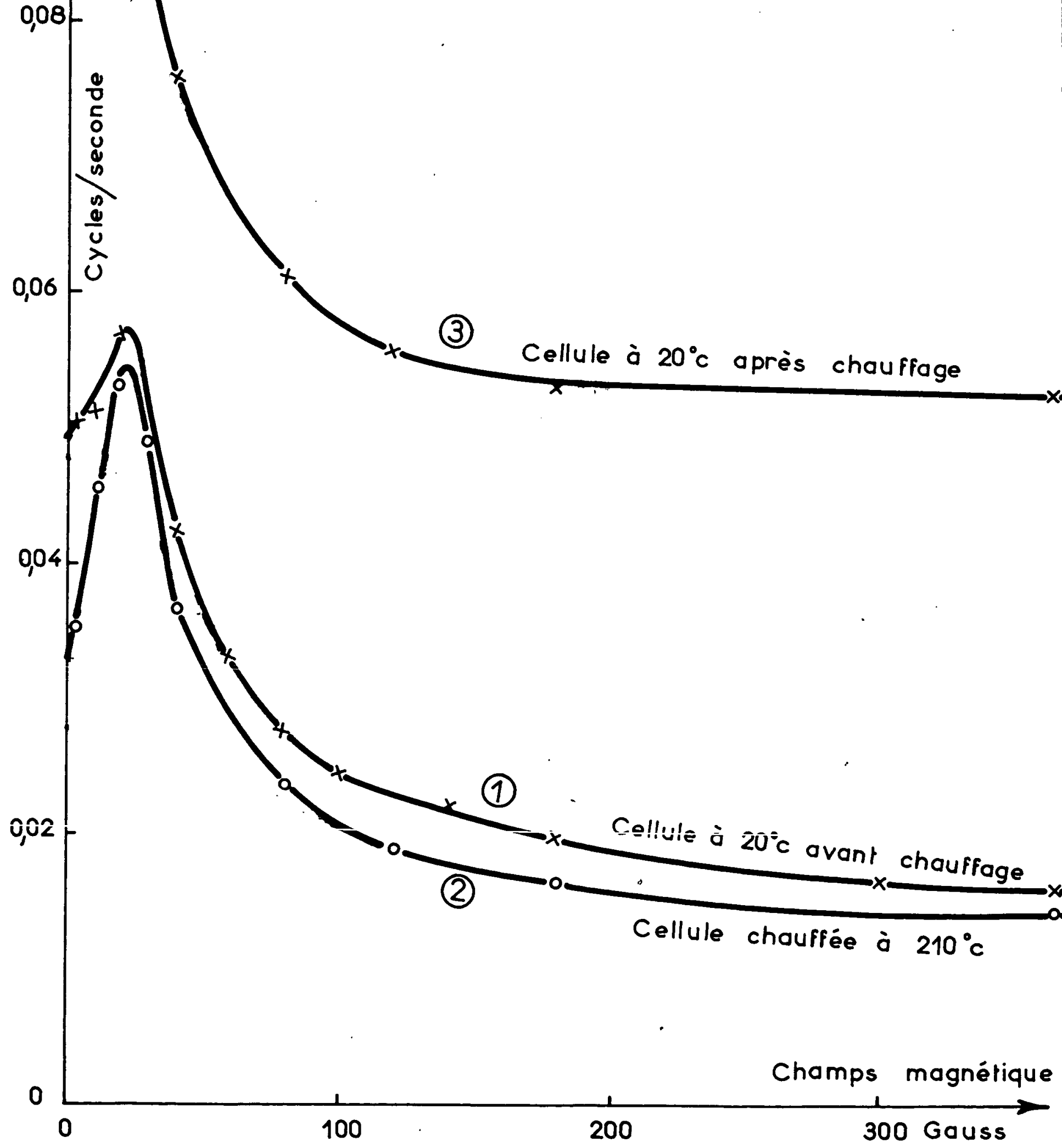